\title{
INFINITESIMAL FORM BOUNDEDNESS AND TRUDINGER'S SUBORDINATION FOR THE SCHRÖDINGER OPERATOR
}

\author{
V. G. MAZ'YA AND I. E. VERBITSKY*
}

ABStract. We give explicit analytic criteria for two problems associated with the Schrödinger operator $H=-\Delta+Q$ on $L^{2}\left(\mathbb{R}^{n}\right)$ where $Q \in D^{\prime}\left(\mathbb{R}^{n}\right)$ is an arbitrary real- or complex-valued potential.

First, we obtain necessary and sufficient conditions on $Q$ so that the quadratic form $\langle Q \cdot, \cdot\rangle$ has zero relative bound with respect to the Laplacian. For $Q \in L_{\mathrm{loc}}^{1}\left(\mathbb{R}^{n}\right)$, this property can be expressed in the form of the integral inequality:

$$
\left.\left|\int_{\mathbb{R}^{n}}\right| u(x)\right|^{2} Q(x) d x \mid \leq \epsilon\|\nabla u\|_{L^{2}\left(\mathbb{R}^{n}\right)}^{2}+C(\epsilon)\|u\|_{L^{2}\left(\mathbb{R}^{n}\right)}^{2}, \quad \forall u \in C_{0}^{\infty}\left(\mathbb{R}^{n}\right),
$$

for an arbitrarily small $\epsilon>0$ and some $C(\epsilon)>0$. One of the major steps here is the reduction to a similar inequality with nonnegative function $\left|\nabla(1-\Delta)^{-1} Q\right|^{2}+\left|(1-\Delta)^{-1} Q\right|$ in place of $Q$. This provides a complete solution to the infinitesimal form boundedness problem for the Schrödinger operator, and leads to new broad classes of admissible distributional potentials $Q$, which extend the usual $L^{p}$ and Kato classes, as well as those based on the well-known conditions of Fefferman-Phong and Chang-Wilson-Wolff.

Secondly, we characterize Trudinger's subordination property where $C(\epsilon)$ in the above inequality is subject to the condition $C(\epsilon) \leq c \epsilon^{-\beta}(\beta>0)$ as $\epsilon \rightarrow+0$. Such quadratic form inequalities can be understood entirely in the framework of Morrey-Campanato spaces, using mean oscillations of $\nabla(1-\Delta)^{-1} Q$ and $(1-\Delta)^{-1} Q$ on balls or cubes. A version of this condition where $\epsilon \in(0,+\infty)$ is equivalent to the multiplicative inequality:

$$
\left.\left|\int_{\mathbb{R}^{n}}\right| u(x)\right|^{2} Q(x) d x \mid \leq C\|\nabla u\|_{L^{2}\left(\mathbb{R}^{n}\right)}^{2 p}\|u\|_{L^{2}\left(\mathbb{R}^{n}\right)}^{2(1-p)}, \quad \forall u \in C_{0}^{\infty}\left(\mathbb{R}^{n}\right),
$$

with $p=\frac{\beta}{1+\beta} \in(0,1)$. We show that this inequality holds if and only if $\nabla \Delta^{-1} Q \in \operatorname{BMO}\left(\mathbb{R}^{n}\right)$ if $p=\frac{1}{2}$. For $0<p<\frac{1}{2}$, it is valid whenever $\nabla \Delta^{-1} Q$ is Hölder-continuous of order $1-2 p$, or respectively lies in the Morrey space $\mathcal{L}^{2, \lambda}$ with $\lambda=n+2-4 p$ if $\frac{1}{2}<p<1$. As a consequence, we characterize completely the class of those $Q$ which satisfy an analogous multiplicative inequality of Nash's type, with $\|u\|_{L^{1}\left(\mathbb{R}^{n}\right)}$ in place of $\|u\|_{L^{2}\left(\mathbb{R}^{n}\right)}$.

These results are intimately connected with spectral theory and dynamics of the Schrödinger operator, and elliptic PDE theory.

\footnotetext{
${ }^{*}$ Supported in part by NSF Grant DMS-0070623.
} 


\section{INTRODUCTION}

In the present paper we obtain necessary and sufficient conditions for the infinitesimal form boundedness of the potential energy operator $Q$ with respect to the kinetic energy operator $H_{0}=-\Delta$ on $L^{2}\left(\mathbb{R}^{n}\right)$. Here $Q$ is an arbitrary real- or complex-valued potential (possibly a distribution). This notion appeared in relation to the so-called KLMN theorem ([RSi], Theorem X.17), and has become an indispensable tool in mathematical quantum mechanics and PDE theory. Furthermore, we characterize explicitly a related form subordination property of Trudinger type. (See Ka2, RSi, [RSS, Sch, Tru, and the literature cited there.)

Both of these notions play a major role in studies of asymptotics of the spectral counting function for self-adjoint and non-self-adjoint Schrödinger operators BiSo, Gr1], Gr2, [MM1, MM2, generalized eigenvector expansions Agr, RSS, Schrödinger semigroups Dav1, Dav2, [LPS, [Sim, stochastic processes and elliptic PDE [AiSi], CZh], [Tru]. Nevertheless, a complete analytic characterization of the corresponding classes of admissible potentials has been an open problem until now.

More precisely, we characterize the class of potentials $Q \in \mathcal{D}^{\prime}\left(\mathbb{R}^{n}\right)$ which are $-\Delta$-form bounded with relative bound zero, i.e., for every $\epsilon>0$, there exists $C(\epsilon)>0$ such that

$$
|\langle Q u, u\rangle| \leq \epsilon|| \nabla u\left\|_{L^{2}\left(\mathbb{R}^{n}\right)}^{2}+C(\epsilon)\right\| u \|_{L^{2}\left(\mathbb{R}^{n}\right)}^{2}, \quad \forall u \in C_{0}^{\infty}\left(\mathbb{R}^{n}\right) .
$$

The preceding inequality ensures that, in case $Q$ is real-valued, a semibounded self-adjoint Schrödinger operator $H=H_{0}+Q$ can be defined on $L^{2}\left(\mathbb{R}^{n}\right)$ so that the quadratic form domain $Q(H)$ coincides with $Q\left(H_{0}\right)=$ $W^{1,2}\left(\mathbb{R}^{n}\right)$. For complex-valued $Q$, it follows that $H$ is an $m$-sectorial operator on $L^{2}\left(\mathbb{R}^{n}\right)$ with $\mathcal{D}(H) \subset W^{1,2}\left(\mathbb{R}^{n}\right)([\mathrm{RSi}]$, Sec. X.2; [EE], Sec. IV.4).

Our characterization of (1.1) uses only the functions $\left|\nabla(1-\Delta)^{-1} Q\right|$ and $\left|(1-\Delta)^{-1} Q\right|$ (see Sec. 2. Theorem I), and is based on the representation:

$$
Q=\operatorname{div} \vec{\Gamma}+\gamma, \quad \vec{\Gamma}(x)=-\nabla(1-\Delta)^{-1} Q, \quad \gamma=(1-\Delta)^{-1} Q .
$$

In particular, we will demonstrate that, necessarily, $\vec{\Gamma} \in L_{\text {loc }}^{2}\left(\mathbb{R}^{n}\right)^{n}, \quad \gamma \in$ $L_{\mathrm{loc}}^{1}\left(\mathbb{R}^{n}\right)$, and, when $n \geq 3$,

$$
\lim _{\delta \rightarrow+0} \sup _{x_{0} \in \mathbb{R}^{n}} \delta^{2-n} \int_{B_{\delta}\left(x_{0}\right)}\left(|\vec{\Gamma}(x)|^{2}+|\gamma(x)|\right) d x=0,
$$

once (1.1) holds. Here $B_{\delta}\left(x_{0}\right)$ is a Euclidean ball of radius $\delta$ centered at $x_{0}$.

In the opposite direction, it follows from our results that (1.1) holds whenever

$$
\lim _{\delta \rightarrow+0} \sup _{x_{0} \in \mathbb{R}^{n}} \delta^{2 r-n} \int_{B_{\delta}\left(x_{0}\right)}\left(|\vec{\Gamma}(x)|^{2}+|\gamma(x)|\right)^{r} d x=0
$$


where $r>1$. Such admissible potentials form a natural analogue of the Fefferman-Phong class [Fef] for the infinitesimal form boundedness problem, where cancellations between the positive and negative parts of $Q$ come into play. It includes functions with highly oscillatory behavior as well as singular measures, and contains properly the class of potentials based on the original Fefferman-Phong condition where $|Q|$ is used in (1.4) in place of $|\vec{\Gamma}|^{2}+|\gamma|$. Moreover, one can expand this class further using a sharp condition due to Chang, Wilson, and Wolff [ChWW] applied to $|\vec{\Gamma}|^{2}+|\gamma|$.

A complete characterization of (1.1) given below involves discrete Carleson measure sums over dyadic cubes, together with equivalent local energy and pointwise potential conditions (Sec. 2, Theorem II). In the proofs given in Sec. 5 we overcome considerable technical difficulties using a series of new crucial estimates for powers of equilibrium potentials, factorization of functions in Sobolev spaces, and theory of $A_{p}$-weights, along with appropriate localization arguments, and good understanding of trace inequalities for nonnegative potentials $Q$ studied respectively in Sec. 3 and Sec. 4.

Among well-known sufficient conditions for (1.1), which ignore possible cancellations, we mention: $Q \in L^{\frac{n}{2}}\left(\mathbb{R}^{n}\right)+L^{\infty}\left(\mathbb{R}^{n}\right)(n \geq 3)$ and $Q \in L^{r}\left(\mathbb{R}^{2}\right)+$ $L^{\infty}\left(\mathbb{R}^{2}\right), r>1(n=2)$ (see BrK), as well as Kato's condition $K_{n}$ introduced in Ka1:

$$
\begin{aligned}
& \lim _{\delta \rightarrow+0} \sup _{x_{0} \in \mathbb{R}^{n}} \int_{B_{\delta}\left(x_{0}\right)} \frac{|Q(x)|}{\left|x-x_{0}\right|^{n-2}} d x=0, \quad n \geq 3, \\
& \lim _{\delta \rightarrow+0} \sup _{x_{0} \in \mathbb{R}^{n}} \int_{B_{\delta}\left(x_{0}\right)} \log \frac{1}{\left|x-x_{0}\right|}|Q(x)| d x=0, \quad n=2 .
\end{aligned}
$$

Kato's class proved to be especially important in studies of Schrödinger semigroups, Dirichlet forms, and Harnack inequalities Agm, AiSi, Sim. Our results yield that actually (1.1) holds for a substantially broader class of potentials for which $|\vec{\Gamma}|^{2}+|\gamma| \in K_{n}$.

We emphasize that no a priori assumptions were imposed above on $C(\epsilon)$. An observation due to Aizenman and Simon states that, under the hypothesis $C(\epsilon) \leq a e^{b \epsilon^{-p}}$ for some $a, b>0$ and $0<p<1$, all potentials $Q$ which obey (1.1) with $|Q|$ in place of $Q$, are contained in Kato's class. This was first proved in AiSi using the Feynman-Kac formalism. In Sec. 4, we give a sharp result of this kind with a simple analytic proof. We show that if (1.1) holds with $|Q|$ in place of $Q \in L_{\text {loc }}^{1}\left(\mathbb{R}^{n}\right)$, then for any $C(\epsilon)>0$,

$$
\begin{aligned}
& \sup _{x_{0} \in \mathbb{R}^{n}} \int_{B_{\delta}\left(x_{0}\right)} \frac{|Q(x)|}{\left|x-x_{0}\right|^{n-2}} d x \leq c \int_{\delta^{-2}}^{+\infty} \frac{\hat{C}(s)}{s^{2}} d s, \quad n \geq 3, \\
& \sup _{x_{0} \in \mathbb{R}^{2}} \int_{B_{\delta}\left(x_{0}\right)} \log \frac{1}{\left|x-x_{0}\right|}|Q(x)| d x \leq c \int_{\delta^{-2}}^{+\infty} \frac{\hat{C}(s)}{s^{2} \log s} d s, \quad n=2,
\end{aligned}
$$


where $c$ is a constant which depends only on $n$, and $\delta$ is sufficiently small. Here $\hat{C}(s)=\inf _{\epsilon>0}\{C(\epsilon)+s \epsilon\}$ is the Legendre transform of $-C(\epsilon)$. In particular, it follows that the condition $C(\epsilon) \leq a e^{b \epsilon^{-p}}$ for any $p>0$ is enough to ensure that $Q \in K_{2}$ in the more subtle two-dimensional case.

In the second part of the paper, we study quadratic form inequalities of Trudinger type where $C(\epsilon)$ in (1.1) has power growth, i.e., there exists $\epsilon_{0}>0$ such that

$$
|\langle Q u, u\rangle| \leq \epsilon\|\nabla u\|_{L^{2}\left(\mathbb{R}^{n}\right)}^{2}+c \epsilon^{-\beta}\|u\|_{L^{2}\left(\mathbb{R}^{n}\right)}^{2}, \quad \forall u \in C_{0}^{\infty}\left(\mathbb{R}^{n}\right),
$$

for every $\epsilon \in\left(0, \epsilon_{0}\right)$, where $\beta>0$. Such inequalities appear in studies of elliptic PDE with measurable coefficients [Tru], and have been used extensively in spectral theory of the Schrödinger operator (see, e.g., [RSS], Sec. 20).

As it turns out, it is still possible to characterize (1.9) using only $|\vec{\Gamma}|$ and $|\gamma|$ defined by (1.2), provided $\beta>1$. We will show below (Theorem IV) that in this case (1.9) holds if and only if both of the following conditions hold:

$$
\begin{aligned}
& \sup _{\substack{x_{0} \in \mathbb{R}^{n} \\
0<\delta<\delta_{0}}} \delta^{2 \frac{\beta-1}{\beta+1}-n} \int_{B_{\delta}\left(x_{0}\right)}|\vec{\Gamma}(x)|^{2} d x<+\infty, \\
& \sup _{\substack{x_{0} \in \mathbb{R}^{n} \\
0<\delta<\delta_{0}}} \delta^{\frac{2 \beta}{\beta+1}-n} \int_{B_{\delta}\left(x_{0}\right)}|\gamma(x)| d x<+\infty,
\end{aligned}
$$

for some $\delta_{0}>0$. However, in the case $\beta \leq 1$ this is no longer true. For $\beta=1$, (1.10) has to be replaced with the condition that $\vec{\Gamma}$ is in the local BMO space, or respectively is Hölder-continuous of order $\frac{1-\beta}{1+\beta}$ if $0<\beta<1$.

In the homogeneous case $\epsilon_{0}=+\infty$, (1.9) is equivalent to the multiplicative inequality:

$$
|\langle Q u, u\rangle| \leq C\|\nabla u\|_{L^{2}\left(\mathbb{R}^{n}\right)}^{2 p}\|u\|_{L^{2}\left(\mathbb{R}^{n}\right)}^{2(1-p)}, \quad \forall u \in C_{0}^{\infty}\left(\mathbb{R}^{n}\right),
$$

where $p=\frac{\beta}{1+\beta} \in(0,1)$. In spectral theory, (1.12) is referred to as the form $p$-subordination property Agr, Gr1, Gr2, [MM1, RSS].

For nonnegative potentials $Q$, where $Q$ coincides with a locally finite measure $\mu$ on $\mathbb{R}^{n}$, inequality (1.12) is known to hold if and only if

$$
\mu\left(B_{\delta}\left(x_{0}\right)\right) \leq c \delta^{n-2 p},
$$

where the constant $c$ does not depend on $\delta>0$ and $x_{0} \in \mathbb{R}^{n}$ ([M2], Sec. 1.4.7).

For general $Q$, we obtain the following result (Theorem $\mathrm{V}$ ): If $p>\frac{1}{2}$, then (1.12) holds if and only if $\nabla \Delta^{-1} Q$ lies in the Morrey space $\mathcal{L}^{2, \lambda}\left(\mathbb{R}^{n}\right)$, where $\lambda=n+2-4 p$. For $p=\frac{1}{2}$, it holds if and only if $\nabla \Delta^{-1} Q \in \operatorname{BMO}\left(\mathbb{R}^{n}\right)$, and for $0<p<\frac{1}{2}$, whenever $\nabla \Delta^{-1} Q \in \operatorname{Lip}_{1-2 p}\left(\mathbb{R}^{n}\right)$. These different characterizations are equivalent to (1.13) if $Q$ is a nonnegative measure. 
As a consequence, we are able to characterize those $Q$ which obey an analogous inequality of Nash's type:

$$
|\langle Q u, u\rangle| \leq C\|\nabla u\|_{L^{2}\left(\mathbb{R}^{n}\right)}^{2 p}\|u\|_{L^{1}\left(\mathbb{R}^{n}\right)}^{2(1-p)}, \quad \forall u \in C_{0}^{\infty}\left(\mathbb{R}^{n}\right) .
$$

where $p \in(0,1)$. In fact, the preceding inequality has two critical exponents, $p_{*}=\frac{n}{n+2}$ and $p^{*}=\frac{n+1}{n+2}$. We will show in Sec. 6 (Corollary6.8) that, for $0<p<$ $p_{*}$, (1.14) holds only if $Q=0$, whereas for $p=p_{*}$ it follows that $Q \in L^{\infty}\left(\mathbb{R}^{n}\right)$, i.e., it is equivalent to Nash's inequality ([LL], Theorem 8.13). For $p>p_{*}$, the validity of (1.14) is equivalent respectively to: $\nabla \Delta^{-1} Q \in \operatorname{Lip}_{n+1-p(n+2)}\left(\mathbb{R}^{n}\right)$ if $p_{*}<p<p^{*} ; \nabla \Delta^{-1} Q \in \operatorname{BMO}\left(\mathbb{R}^{n}\right)$ if $p=p^{*}$; and $\nabla \Delta^{-1} Q \in \mathcal{L}^{2, \lambda}\left(\mathbb{R}^{n}\right)$, where $\lambda=3 n+2-2 p(n+2)$, if $p^{*}<p<1$.

There is an interesting connection of our form subordination theorems, in the sufficiency part, with estimates of the type:

$$
\|(\vec{u} \cdot \nabla) \vec{u}\|_{\mathcal{H}^{1}\left(\mathbb{R}^{n}\right)} \leq c\|\vec{u}\|_{L^{2}\left(\mathbb{R}^{n}\right)}\|\nabla \vec{u}\|_{L^{2}\left(\mathbb{R}^{n}\right)}, \quad \operatorname{div} \vec{u}=\overrightarrow{0}, \quad \forall \vec{u} \in C_{0}^{\infty}\left(\mathbb{R}^{n}\right)^{n},
$$

where $\mathcal{H}^{1}\left(\mathbb{R}^{n}\right)$ is a real Hardy space $([\mathrm{St} 2])$. Such inequalities and their generalizations considered in Sec. 6] (see Lemma 6.6) are useful in hydrodynamics (CLMS, CO ).

Our proofs of the necessity statements are generally more delicate than those of the sufficiency. They make use of factorization of functions in Sobolev spaces, and sharp mean oscillation estimates for "anti-derivatives" of $Q$. Our methods based on a combination of ideas from potential theory, Sobolev spaces, nonlinear and harmonic analyses have been developed over the past ten years HMV], MV1]-MV4. We believe that this approach has a broader scope, and might be useful with regards to higher order elliptic operators, pseudodifferential operators, harmonic analysis and nonlinear PDE problems (see, e.g., [BoBr], Dav1], [Dav2], [Fef]).

We conclude the introduction with a brief outline of the contents of the paper. In Sec. 2, we introduce some notation and state our main results, Theorems I-V. In Sec. 3, we present a useful localization principle for quadratic form inequalities using a Legendre transform associated with the function $C(\epsilon)$. This localization will be employed throughout the paper. Sec. 4 is devoted to a study of the corresponding integral inequalities for nonnegative potentials $Q$ (locally finite measures). In particular, we discuss connections with Kato's condition which is important to Schrödinger operators and elliptic PDE.

The proofs of the main results are given in Sections 5 and 6 where we treat general distributional potentials $Q$. In Sec. 5], we establish necessary and sufficient conditions for $-\Delta$-form boundedness of the potential energy operator $Q$ with relative bound zero. In Sec. 6, we obtain subordination criteria for quadratic forms associated with the Schrödinger operator, and discuss connections with the compensated compactness phenomenon. 


\section{MAin RESUlts}

Throughout the paper we will be using the following notation and conventions. We denote by $W^{1,2}\left(\mathbb{R}^{n}\right)$ the Sobolev space of weakly differentiable functions on $\mathbb{R}^{n}(n \geq 1)$ such that

$$
\|u\|_{W^{1,2}\left(\mathbb{R}^{n}\right)}=\|u\|_{L^{2}\left(\mathbb{R}^{n}\right)}+\|\nabla u\|_{L^{2}\left(\mathbb{R}^{n}\right)}<+\infty,
$$

and by $W^{-1,2}\left(\mathbb{R}^{n}\right)=W^{1,2}\left(\mathbb{R}^{n}\right)^{*}$ the dual Sobolev space. For a compact set $e \subset \mathbb{R}^{n}$, the capacity associated with $W^{1,2}\left(\mathbb{R}^{n}\right)$ is defined by

$$
\operatorname{cap}(e)=\inf \left\{\|u\|_{W^{1,2}\left(\mathbb{R}^{n}\right)}^{2}: \quad u \in C_{0}^{\infty}\left(\mathbb{R}^{n}\right), \quad u(x)>1 \text { on } e\right\} .
$$

For $0<r<\infty$, we denote by $L_{\text {unif }}^{r}\left(\mathbb{R}^{n}\right)$ all $f \in L_{\text {loc }}^{r}\left(\mathbb{R}^{n}\right)$ such that

$$
\|f\|_{L_{\text {unif }}^{r}}=\sup _{x_{0} \in \mathbb{R}^{n}}\left\|\chi_{B_{1}\left(x_{0}\right)} f\right\|_{L^{r}\left(\mathbb{R}^{n}\right)}<\infty .
$$

By $L^{r}\left(\mathbb{R}^{n}\right)^{n}$ we denote the class of vector fields $\vec{\Gamma}=\left\{\Gamma_{j}\right\}_{j=1}^{n}: \mathbb{R}^{n} \rightarrow \mathbb{C}^{n}$, such that $\Gamma_{j} \in L^{r}\left(\mathbb{R}^{n}\right), j=1,2, \ldots, n$, and use similar notation for other vector-valued function spaces.

By $M^{+}\left(\mathbb{R}^{n}\right)$ we denote the class of nonnegative locally finite Borel measures on $\mathbb{R}^{n}$. If $Q \in \mathcal{D}^{\prime}\left(\mathbb{R}^{n}\right)$ is nonnegative, i.e., coincides with $\mu \in M^{+}\left(\mathbb{R}^{n}\right)$, we write $\int_{\mathbb{R}^{n}}|u(x)|^{2} d \mu$ in place of $\left\langle Q,|u|^{2}\right\rangle=\langle Q u, u\rangle$ for the quadratic form associated with the distribution $Q$, if $u \in C_{0}^{\infty}\left(\mathbb{R}^{n}\right)$. Sometimes we will use $\int_{\mathbb{R}^{n}}|u(x)|^{2} Q(x) d x$ in place of $\langle Q u, u\rangle$ even if $Q$ is not in $L_{\text {loc }}^{1}\left(\mathbb{R}^{n}\right)$.

We set

$$
m_{B}(f)=\frac{1}{|B|} \int_{B} f(x) d x
$$

for a ball $B \subset \mathbb{R}^{n}$, and denote by $\operatorname{BMO}\left(\mathbb{R}^{n}\right)$ the class of $f \in L_{\mathrm{loc}}^{r}\left(\mathbb{R}^{n}\right)$ for which

$$
\sup _{x_{0} \in \mathbb{R}^{n}, \delta>0} \frac{1}{\left|B_{\delta}\left(x_{0}\right)\right|} \int_{B_{\delta}\left(x_{0}\right)}\left|f(x)-m_{B_{\delta}\left(x_{0}\right)}(f)\right|^{r} d x<+\infty,
$$

for any (or, equivalently, all) $1 \leq r<+\infty$.

We will also need an inhomogeneous version of $\mathrm{BMO}\left(\mathbb{R}^{n}\right)$ (the so-called local BMO) which we denote by $\operatorname{bmo}\left(\mathbb{R}^{n}\right)$. It can be defined in a similar way as the set of $f \in L_{\text {unif }}^{r}\left(\mathbb{R}^{n}\right)$ such that the preceding condition holds for $0<\delta \leq 1$ (see St2, p. 264).

The Morrey space $\mathcal{L}^{r, \lambda}(r>0, \lambda>0)$ consists of $f \in L_{\mathrm{loc}}^{r}\left(\mathbb{R}^{n}\right)$ such that

$$
\sup _{x_{0} \in \mathbb{R}^{n}, \delta>0} \frac{1}{\left|B_{\delta}\left(x_{0}\right)\right|^{1-\frac{\lambda}{n}}} \int_{B_{\delta}\left(x_{0}\right)}|f|^{r} d x<+\infty .
$$

In the corresponding inhomogeneous analogue, we set $0<\delta \leq 1$ in the preceding inequality. It will be clear from the context which version of the Morrey space is used.

We now state our main results. 
Theorem I. Let $Q \in \mathcal{D}^{\prime}\left(\mathbb{R}^{n}\right), n \geq 2$. Then the following statements hold.

(i) Suppose that $Q$ is represented in the form:

$$
Q=\operatorname{div} \vec{\Gamma}+\gamma
$$

where $\vec{\Gamma} \in L_{\text {loc }}^{2}\left(\mathbb{R}^{n}\right)^{n}$ and $\gamma \in L_{\text {loc }}^{1}\left(\mathbb{R}^{n}\right)$ satisfy respectively the conditions:

$$
\begin{aligned}
& \lim _{\delta \rightarrow+0} \sup _{x_{0} \in \mathbb{R}^{n}} \sup _{u} \frac{\int_{B_{\delta}\left(x_{0}\right)}|\vec{\Gamma}(x)|^{2}|u(x)|^{2} d x}{\|\left.\nabla u\right|_{L^{2}\left(B_{\delta}\left(x_{0}\right)\right)} ^{2}}=0, \\
& \lim _{\delta \rightarrow+0} \sup _{x_{0} \in \mathbb{R}^{n}} \sup _{u} \frac{\int_{B_{\delta}\left(x_{0}\right)}|\gamma(x)||u(x)|^{2} d x}{\|\left.\nabla u\right|_{L^{2}\left(B_{\delta}\left(x_{0}\right)\right)} ^{2}}=0,
\end{aligned}
$$

where $u \in C_{0}^{\infty}\left(B_{\delta}\left(x_{0}\right)\right), u \not \equiv 0$. Then $Q$ is infinitesimally form bounded with respect to $-\Delta$, i.e., for every $\epsilon>0$ there exists $C(\epsilon)>0$ such that (1.1) holds.

(ii) Conversely, suppose $Q$ is infinitesimally form bounded with respect to $-\Delta$. Then $Q$ can be represented in the form (2.1) so that both (2.2) and (2.3) hold. Moreover, one can set $\vec{\Gamma}=-\nabla(1-\Delta)^{-1} Q$ and $\gamma=(1-\Delta)^{-1} Q$ in the representation (2.1).

Remark 2.1. In the statement of Theorem I one can replace conditions (2.2) and (2.3) with the equivalent condition where $\left|(1-\Delta)^{-\frac{1}{2}} Q\right|^{2}$ is used in place of $|\vec{\Gamma}|^{2}$ in (2.2).

The importance of Theorem I is in the means it provides for deducing explicit criteria of the infinitesimal form boundedness in terms of the nonnegative locally integrable functions $|\vec{\Gamma}|^{2}$ and $|\gamma|$.

Theorem II. Let $Q \in \mathcal{D}^{\prime}\left(\mathbb{R}^{n}\right), n \geq 2$. The following statements are equivalent:

(i) $Q$ is infinitesimally form bounded with respect to $-\Delta$.

(ii) $Q$ has the form (2.1) where $\vec{\Gamma}=-\nabla(1-\Delta)^{-1} Q, \gamma=(1-\Delta)^{-1} Q$, and the measure $\mu \in M^{+}\left(\mathbb{R}^{n}\right)$ defined by

$$
d \mu=\left(|\vec{\Gamma}(x)|^{2}+|\gamma(x)|\right) d x
$$

has the property that, for every $\epsilon>0$, there exists $C(\epsilon)>0$ such that

$$
\int_{\mathbb{R}^{n}}|u(x)|^{2} d \mu \leq \epsilon\|\nabla u\|_{L^{2}\left(\mathbb{R}^{n}\right)}^{2}+C(\epsilon)\|\nabla u\|_{L^{2}\left(\mathbb{R}^{n}\right)}^{2}, \quad \forall u \in C_{0}^{\infty}\left(\mathbb{R}^{n}\right) .
$$

(iii) For $\mu$ defined by (2.4),

$$
\lim _{\delta \rightarrow+0} \sup _{P_{0}: \operatorname{diam} P_{0} \leq \delta} \frac{1}{\mu\left(P_{0}\right)} \sum_{P \subseteq P_{0}} \frac{\mu(P)^{2}}{|P|^{1-\frac{2}{n}}}=0,
$$

where $P, P_{0}$ are dyadic cubes in $\mathbb{R}^{n}$. 
(iv) For $\mu$ defined by (2.4),

$$
\lim _{\delta \rightarrow+0} \sup _{e: \operatorname{diam} e \leq \delta} \frac{\mu(e)}{\operatorname{cap}(e)}=0
$$

where e denotes a compact set of positive capacity in $\mathbb{R}^{n}$.

(v) For $\mu$ defined by (2.4),

$$
\lim _{\delta \rightarrow+0} \sup _{x_{0} \in \mathbb{R}^{n}} \frac{\left\|\mu_{B_{\delta}\left(x_{0}\right)}\right\|_{W^{-1,2}\left(\mathbb{R}^{n}\right)}^{2}}{\mu\left(B_{\delta}\left(x_{0}\right)\right)}=0,
$$

where $\mu_{B_{\delta}\left(x_{0}\right)}$ is the restriction of $\mu$ to the ball $B_{\delta}\left(x_{0}\right)$.

(vi) For $\mu$ defined by (2.4),

$$
\lim _{\delta \rightarrow+0} \sup _{x, x_{0} \in \mathbb{R}^{n}} \frac{G_{1} \star\left(G_{1} \star \mu_{B_{\delta}\left(x_{0}\right)}\right)^{2}(x)}{G_{1} \star \mu_{B_{\delta}\left(x_{0}\right)}(x)}=0,
$$

where $G_{1} \star \mu=(1-\Delta)^{-\frac{1}{2}} \mu$ is the Bessel potential of order 1 .

It is worth noting that although Theorem II holds in the two-dimensional case, its proof requires certain modifications in comparison to $n \geq 3$. In the one-dimensional case, the infinitesimal form boundedness of the SturmLiouville operator $H=-\frac{d^{2}}{d x^{2}}+Q$ on $L^{2}\left(\mathbb{R}^{1}\right)$ is actually a consequence of the form boundedness.

Theorem III. Let $Q \in \mathcal{D}^{\prime}\left(\mathbb{R}^{1}\right)$. Then the following statements are equivalent.

(i) $Q$ is infinitesimally form bounded with respect to $-\frac{d^{2}}{d x^{2}}$.

(ii) $Q$ is form bounded with respect to $-\frac{d^{2}}{d x^{2}}$, i.e.,

$$
|\langle Q u, u\rangle| \leq \mathrm{const}\|u\|_{W^{1,2}\left(\mathbb{R}^{1}\right)}^{2}, \quad \forall u \in C_{0}^{\infty}\left(\mathbb{R}^{1}\right) .
$$

(iii) $Q$ can be represented in the form $Q=\frac{d \Gamma}{d x}+\gamma$, where

$$
\sup _{x \in \mathbb{R}^{1}} \int_{x}^{x+1}\left(|\Gamma(x)|^{2}+|\gamma(x)|\right) d x<+\infty .
$$

(iv) Condition (2.10) holds where

$$
\Gamma(x)=\int_{\mathbb{R}^{1}} \operatorname{sign}(x-t) e^{-|x-t|} Q(t) d t, \quad \gamma(x)=\int_{\mathbb{R}^{1}} e^{-|x-t|} Q(t) d t
$$

are understood in the distributional sense.

The statement (iii) $\Rightarrow$ (i) in Theorem III is known ([Sch], Theorem 11.2.1), whereas (ii) $\Rightarrow($ iv) follows from our earlier results MV2, MV3. 
We now state a characterization of the form subordination property (1.9). It was formulated originally in [Tru, in the form of the inequality:

$$
|\langle Q u, u\rangle| \leq \epsilon\|\nabla u\|_{L^{2}\left(\mathbb{R}^{n}\right)}^{2}+c \epsilon^{-\nu}\|u\|_{L^{1}\left(\mathbb{R}^{n}\right)}^{2}, \quad \forall u \in C_{0}^{\infty}\left(\mathbb{R}^{n}\right),
$$

for $Q \geq 0$. Such $Q$ are called $\epsilon^{\nu}$-compactly bounded in [Tru]. It follows from Nash's inequality that (1.9) yields (2.11) with $\nu=\frac{n+2}{2} \beta+\frac{n}{2}$; the converse is also true, provided $\nu>\frac{n}{2}$, and is deduced below using a localization argument (Sec. 3. Corollary 3.3). In the critical case $\nu=\frac{n}{2}$, (2.11) holds if and only if $Q \in L^{\infty}\left(\mathbb{R}^{n}\right)$, while for $0<\nu<\frac{n}{2}$, it holds only if $Q=0$.

Necessary and sufficient conditions for (1.9), or equivalently (2.11) with $\nu=\frac{n+2}{2} \beta+\frac{n}{2}$, can be formulated in terms of Morrey-Campanato spaces using mean oscillations of the functions $\vec{\Gamma}$ and $\gamma$ which have appeared in Theorems I-III.

Theorem IV. Let $Q \in \mathcal{D}^{\prime}\left(\mathbb{R}^{n}\right), n \geq 2$, and let $0<\beta<+\infty$.

(i) Suppose there exists $\epsilon_{0}>0$ such that (1.9) holds for every $\epsilon \in\left(0, \epsilon_{0}\right)$. Then $Q$ can be represented in the form

$$
Q=\operatorname{div} \vec{\Gamma}+\gamma
$$

where $\vec{\Gamma}=-\nabla(1-\Delta)^{-1} Q \in L_{\text {loc }}^{2}\left(\mathbb{R}^{n}\right)^{n}$ and $\gamma=(1-\Delta)^{-1} Q \in L_{\text {loc }}^{1}\left(\mathbb{R}^{n}\right)$.

Moreover, there exists $\delta_{0}>0$ such that

$$
\begin{gathered}
\int_{B_{\delta}\left(x_{0}\right)}\left|\vec{\Gamma}(x)-m_{B_{\delta}\left(x_{0}\right)}(\vec{\Gamma})\right|^{2} d x \leq c \delta^{n-2 \frac{\beta-1}{\beta+1}}, \quad 0<\delta<\delta_{0}, \\
\int_{B_{\delta}\left(x_{0}\right)}|\gamma(x)| d x \leq c \delta^{n-\frac{2 \beta}{\beta+1}}, \quad 0<\delta<\delta_{0}
\end{gathered}
$$

where $c$ does not depend on $x_{0} \in R^{n}$ and $\delta_{0}$. Furthermore, $\vec{\Gamma} \in L_{\text {unif }}^{2}\left(\mathbb{R}^{n}\right)^{n}$ if $\beta \geq 1$ and $\vec{\Gamma} \in L^{\infty}\left(\mathbb{R}^{n}\right)^{n}$ if $0<\beta<1$.

(ii) Conversely, if $Q$ is given by (2.12) where $\vec{\Gamma} \in \mathbf{L}_{\text {loc }}^{2}\left(\mathbb{R}^{n}\right), \gamma \in L_{\text {loc }}^{1}\left(\mathbb{R}^{n}\right)$ satisfy (2.13), (2.14) for all $0<\delta<\delta_{0}$ then there exists $\epsilon_{0}>0$ such that (1.9) holds for all $0<\epsilon<\epsilon_{0}$.

Remark 2.2. (a) In the case $\beta=1$, it follows that (2.13) holds if and only if $\vec{\Gamma} \in \operatorname{bmo}\left(\mathbb{R}^{n}\right)$. In other words, $Q \in$ bmo $_{-1}\left(\mathbb{R}^{n}\right)=F_{-1}^{2, \infty}\left(\mathbb{R}^{n}\right)$, where $F_{\alpha}^{p, q}$ stands for the scale of Triebel-Lizorkin spaces (see, e.g., [AH], [Tri]).

(b) Similarly, in the case $0<\beta<1$, (2.13) holds if and only if $\vec{\Gamma}$ is Höldercontinuous:

$$
\left|\vec{\Gamma}(x)-\vec{\Gamma}\left(x^{\prime}\right)\right| \leq c\left|x-x^{\prime}\right|^{\frac{1-\beta}{\beta+1}}, \quad\left|x-x^{\prime}\right|<\delta_{0}
$$


(c) For $\beta>1$, (2.13) holds if and only if $\vec{\Gamma}$ is in the corresponding Morrey space $\mathcal{L}^{2, n-2 \frac{\beta-1}{\beta+1}}$ :

$$
\int_{B_{\delta}\left(x_{0}\right)}|\vec{\Gamma}(x)|^{2} d x \leq c \delta^{n-2 \frac{\beta-1}{\beta+1}}, \quad 0<\delta<\delta_{0} .
$$

Note that, according to (2.14),$\gamma \in \mathcal{L}^{1, n-\frac{2 \beta}{\beta+1}}$. Equivalently, $Q$ is in the corresponding Campanato-Morrey space $\mathcal{L}_{-1}^{2, n-2 \frac{\beta-1}{\beta+1}}$ of negative order.

Remark 2.3. (a) An immediate consequence of Theorem IV is that, for all $\beta>0$, (1.9) is equivalent to the following localized condition:

$$
\left\|(1-\Delta)^{-\frac{1}{2}}\left(\eta_{\delta, x_{0}} Q\right)\right\|_{L^{2}\left(B_{\delta}\left(x_{0}\right)\right)}^{2} \leq c \delta^{n-2 \frac{\beta-1}{\beta+1}}, \quad 0<\delta<\delta_{0}, x_{0} \in \mathbb{R}^{n},
$$

where $\eta_{\delta, x_{0}}(x)=\eta\left(\frac{\left|x-x_{0}\right|}{\delta}\right)$, and $\eta$ is a smooth cut-off function with compact support.

(b) A similar energy condition,

$$
\left\|(1-\Delta)^{-\frac{1}{2}}\left(\eta_{\delta, x_{0}} Q\right)\right\|_{L^{2}\left(\mathbb{R}^{n}\right)}^{2} \leq c \delta^{n-2 \frac{\beta-1}{\beta+1}}, \quad 0<\delta<\delta_{0}, x_{0} \in \mathbb{R}^{n},
$$

is sufficient but generally not necessary in the case $n=2$.

We next state a criterion for the multiplicative inequality (1.12) to hold, which is equivalent to a homogeneous version of (1.9) with $\epsilon_{0}=+\infty$ and $p=\frac{\beta}{\beta+1}$.

Theorem V. Let $Q \in \mathcal{D}^{\prime}\left(\mathbb{R}^{n}\right), n \geq 2$, and let $0<p<1$.

(i) Suppose that (1.12) holds. Then $Q$ can be represented in the form

$$
Q=\operatorname{div} \vec{\Gamma}
$$

where $\vec{\Gamma}=\nabla \Delta^{-1} Q$, and one of the following conditions hold:

$$
\begin{gathered}
\vec{\Gamma} \in \operatorname{BMO}\left(\mathbb{R}^{n}\right) \quad \text { if } \quad p=\frac{1}{2} ; \quad \vec{\Gamma} \in \operatorname{Lip}_{1-2 p}\left(\mathbb{R}^{n}\right) \quad \text { if } \quad 0<p<\frac{1}{2} ; \\
\int_{B_{\delta}\left(x_{0}\right)}|\vec{\Gamma}(x)|^{2} d x \leq c \delta^{n+2-4 p}, \quad \text { if } \quad \frac{1}{2}<p<1 ;
\end{gathered}
$$

where $c$ does not depend on $x_{0} \in \mathbb{R}^{n}, \delta>0$.

(ii) Conversely, if $Q$ is given by (2.15) where $\vec{\Gamma} \in L_{\text {loc }}^{2}\left(\mathbb{R}^{n}\right)^{n}$ and satisfies (2.16), (2.17) then (1.12) holds.

Remark 2.4. In Theorem $\mathrm{V}$, the "antiderivative" $\vec{\Gamma}=\nabla \Delta^{-1} Q$ can be replaced by $(-\Delta)^{-\frac{1}{2}} Q$. Furthermore, as a corollary we deduce that (1.12) holds if and only if $Q \in \mathrm{BMO}_{-1}\left(\mathbb{R}^{n}\right)=\dot{F}_{-1}^{2, \infty}\left(\mathbb{R}^{n}\right)$ for $p=\frac{1}{2}$, and $Q \in \dot{B}_{-2 p}^{\infty, \infty}\left(\mathbb{R}^{n}\right)$ for 
$0<p<\frac{1}{2}$. Here $\dot{F}_{\alpha}^{r, q}$ and $\dot{B}_{\alpha}^{r, q}$ are homogeneous Triebel-Lizorkin and Besov spaces respectively (see [Tri]).

In the case $p=\frac{1}{2}$, statement (ii) of Theorem $\mathrm{V}$ (sufficiency of the condition $\vec{\Gamma} \in \mathrm{BMO})$ is equivalent via the $\mathcal{H}^{1}-\mathrm{BMO}$ duality to the inequality

$$
\|u \nabla u\|_{\mathcal{H}^{1}\left(\mathbb{R}^{n}\right)} \leq c\|u\|_{L^{2}\left(\mathbb{R}^{n}\right)}\|\nabla u\|_{L^{2}\left(\mathbb{R}^{n}\right)}, \quad \forall u \in C_{0}^{\infty}\left(\mathbb{R}^{n}\right) .
$$

Here $\mathcal{H}^{1}\left(\mathbb{R}^{n}\right)$ is the real Hardy space on $\mathbb{R}^{n}$ ([St2] $)$.

The preceding estimate yields the following vector-valued inequality which is used in studies of the Navier-Stokes equation, and is related to the compensated compactness phenomenon (see [C], CLMS]):

$$
\|(\vec{u} \cdot \nabla) \vec{u}\|_{\mathcal{H}^{1}\left(\mathbb{R}^{n}\right)} \leq\left. c\|\vec{u}\|_{L^{2}\left(\mathbb{R}^{n}\right)}|| \nabla \vec{u}\right|_{L^{2}\left(\mathbb{R}^{n}\right)}, \quad \operatorname{div} \vec{u}=\overrightarrow{0},
$$

for all $\vec{u} \in C_{0}^{\infty}\left(\mathbb{R}^{n}\right)^{n}$.

In Sec. 6] we deduce a nonhomogeneous version of the well-known div-curl lemma CLMS] which yields both (2.18) and (2.19).

\section{LOCALIZATION OF QUADRATIC FORM ESTIMATES}

In this section we restate the infinitesimal form boundedness property (1.1) in an equivalent localized form.

Lemma 3.1. Suppose $Q \in \mathcal{D}^{\prime}\left(\mathbb{R}^{n}\right), n \geq 1$. Then the following statements are equivalent.

(i) There exists a positive constant $\epsilon_{0}$ and a function $C(\epsilon):\left(0, \epsilon_{0}\right) \rightarrow \mathbb{R}_{+}$ such that the inequality

$$
|\langle Q u, u\rangle| \leq \epsilon|| \nabla u\left\|_{L^{2}}^{2}+C(\epsilon)\right\| u \|_{L^{2}}^{2}, \quad \forall u \in C_{0}^{\infty}\left(\mathbb{R}^{n}\right),
$$

holds for every $\epsilon \in\left(0, \epsilon_{0}\right)$.

$$
\lim _{\delta \rightarrow+0} \sup _{x_{0} \in \mathbb{R}^{n}} \sup \left\{|\langle Q u, u\rangle|: u \in C_{0}^{\infty}\left(B_{\delta}\left(x_{0}\right)\right),\|\nabla u\|_{L^{2}} \leq 1\right\}=0 .
$$

Moreover, (3.1) implies that

$$
\sup _{x_{0} \in \mathbb{R}^{n}} \sup \left\{|\langle Q u, u\rangle|: u \in C_{0}^{\infty}\left(B_{\delta}\left(x_{0}\right)\right),\|\nabla u\|_{L^{2}} \leq 1\right\} \leq c(n) \epsilon,
$$

for $\delta=\sqrt{\frac{\epsilon}{C(\epsilon)}}$, where the constant $c(n)$ depends only on $n$.

Conversely, if the preceding inequality holds with $\delta=\sqrt{\frac{\epsilon}{C(\epsilon)}}$ and (sufficiently small) constant $c(n)$ then (3.1) holds.

Proof. Suppose (3.1) holds. As was mentioned above, we can assume without loss of generality that $\epsilon_{0}=+\infty$. Let $\delta>0, x_{0} \in \mathbb{R}^{n}$, and let $u \in C_{0}^{\infty}\left(B_{\delta}\left(x_{0}\right)\right)$. Then clearly

$$
\|u\|_{L^{2}} \leq k(n) \delta\|\nabla u\|_{L^{2}}
$$


where the constant $k(n)$ depends only on $n$. Hence

$$
|\langle Q u, u\rangle| \leq\left(\epsilon+k(n)^{2} \delta^{2} C(\epsilon)\right)\|\nabla u\|_{L^{2}}^{2}, \quad \forall u \in C_{0}^{\infty}\left(B_{\delta}\left(x_{0}\right)\right),
$$

which implies

$$
\begin{aligned}
\sup _{x_{0} \in \mathbb{R}^{n}} & \sup \left\{|\langle Q u, u\rangle|: u \in C_{0}^{\infty}\left(B_{\delta}\left(x_{0}\right)\right),\|\nabla u\|_{L^{2}} \leq 1\right\} \\
& \leq \epsilon+k(n)^{2} \delta^{2} C(\epsilon) .
\end{aligned}
$$

Letting $\delta \rightarrow+0$, and then $\epsilon \rightarrow+0$, in the preceding inequality, we obtain (3.2).

Conversely, if (3.2) holds then, for every $\epsilon>0$ there exists $\delta=\delta(\epsilon)$ so that, for every $x_{0} \in \mathbb{R}^{n}$,

$$
|\langle Q v, v\rangle| \leq \epsilon|| \nabla v \|_{L^{2}\left(\mathbb{R}^{n}\right)}^{2}, \quad \forall v \in C_{0}^{\infty}\left(B_{\delta}\left(x_{0}\right)\right) .
$$

Now fix $u \in C_{0}^{\infty}\left(\mathbb{R}^{n}\right)$ which is supported in $B_{R}(0)$. Let $\eta \in C_{0}^{\infty}\left(B_{1}(0)\right)$ be a cut-off function such that $0 \leq \eta(x) \leq 1, \eta(x)=1$ for $|x| \leq \frac{1}{2},|\nabla \eta(x)| \leq c(n)$, and let $\eta_{\delta, x_{0}}(x)=\eta\left(\frac{x-x_{0}}{\delta}\right)$.

Next, let us pick $x_{i} \in \mathbb{R}^{n}(i=1,2, \ldots)$ so that $\left\{x_{i}\right\}$ form a cubic lattice with grid distance $\frac{\delta}{2 \sqrt{n}}$, and let $\eta_{i}(x)=\eta\left(\frac{x-x_{i}}{\delta}\right)$. Let

$$
\phi(x)=\sum_{i} \eta_{i}^{2}(x)
$$

where the sum is taken over a finite number of indices $i$ such that $B_{2 R}(0) \subset$ $\cup B_{\delta}\left(x_{i}\right)$. Notice that

$$
1 \leq \phi(x) \leq \kappa_{1}(n) \quad \text { on } \quad B_{2 R}(0),
$$

and

$$
|\nabla \phi(x)| \leq \frac{\kappa_{2}(n)}{\delta} \quad \text { on } \quad B_{2 R}(0)
$$

Define

$$
\zeta_{i}(x)=\frac{\eta_{i}(x)}{\sqrt{\phi(x)}}
$$

so that

$$
\sum_{i} \zeta_{i}^{2}(x) \equiv 1 \quad \text { on } \quad B_{R}(0) .
$$

Then by (3.6),

$$
\begin{aligned}
|\langle Q u, u\rangle| & =\left|\sum_{i}\left\langle Q,\left|\zeta_{i} u\right|^{2}\right\rangle\right| \\
& \leq \sum_{i}\left|\left\langle Q,\left|\zeta_{i} u\right|^{2}\right\rangle\right|
\end{aligned}
$$




$$
\begin{aligned}
& \leq \epsilon \sum_{i} \int|\nabla u|^{2} \zeta_{i}^{2} d x+\epsilon \sum_{i} \int|u|^{2}\left|\nabla \zeta_{i}\right|^{2} d x \\
& \leq \epsilon\|\nabla u\|_{L^{2}}^{2}+c(n) \epsilon \delta^{-2}\|u\|_{L^{2}}^{2} .
\end{aligned}
$$

In the last line we have used the estimate

$$
\sum_{i}\left|\nabla \zeta_{i}(x)\right|^{2} \leq \frac{c(n)}{\delta^{2}} \frac{1}{\phi(x)}+\frac{|\nabla \phi(x)|^{2}}{\phi(x)^{2}} \leq c(n) \delta^{-2}, \quad x \in B_{R}(0) .
$$

Setting $C(\epsilon)=c(n) \epsilon \delta(\epsilon)^{-2}$, we get :

$$
|\langle Q u, u\rangle| \leq \epsilon|| \nabla u\left\|_{L^{2}\left(\mathbb{R}^{n}\right)}^{2}+C(\epsilon)\right\| u \|_{L^{2}\left(\mathbb{R}^{n}\right)}^{2} .
$$

This proves (3.1).

Let $0<\epsilon_{0} \leq+\infty$, and let $C(\epsilon):\left(0, \epsilon_{0}\right) \rightarrow \mathbb{R}^{+}$. We define a modified Legendre transform by:

$$
\hat{C}(s)=\inf _{\epsilon \in\left(0, \epsilon_{0}\right)}\{\epsilon s+C(\epsilon)\}, \quad s>0,
$$

Note that $\hat{C}(s)$ coincides with the Legendre transform of $-C(\epsilon)$. Obviously, $\hat{C}(s)$ is increasing, concave, and $\hat{C}(2 s) \leq 2 \hat{C}(s)(s>0)$. The following statement is an immediate consequence of (3.5).

Corollary 3.2. Suppose that (3.1) holds. Then

$$
\begin{aligned}
& \sup _{x_{0} \in \mathbb{R}^{n}} \sup \left\{|\langle Q u, u\rangle|: u \in C_{0}^{\infty}\left(B_{\delta}\left(x_{0}\right)\right),\|\nabla u\|_{L^{2}} \leq 1\right\} \\
& \leq k(n) \delta^{2} \hat{C}\left(\delta^{-2}\right),
\end{aligned}
$$

where $k(n)$ is a constant which depends only on $n$.

In the special case where $C(\epsilon)=c \epsilon^{-\beta}$ and consequently $\hat{C}(s)=c_{1} s^{\frac{\beta}{\beta+1}}$, we have the following characterization of Trudinger's subordination property.

Corollary 3.3. Suppose $Q \in D^{\prime}\left(\mathbb{R}^{n}\right), n \geq 2$. Suppose $\beta>0$ and $\nu=\frac{n+2}{2} \beta+\frac{n}{2}$. Then the following statements are equivalent.

(i) There exist $\epsilon_{0}>0$ and $c>0$ such that, for every $\epsilon \in\left(0, \epsilon_{0}\right)$,

$$
|\langle Q u, u\rangle| \leq \epsilon|| \nabla u\left\|_{L^{2}\left(\mathbb{R}^{n}\right)}^{2}+c \epsilon^{-\beta}\right\| u \|_{L^{2}\left(\mathbb{R}^{n}\right)}^{2}, \quad \forall u \in C_{0}^{\infty}\left(\mathbb{R}^{n}\right) .
$$

(ii) There exist $\epsilon_{0}>0$ and $c>0$ such that, for every $\epsilon \in\left(0, \epsilon_{0}\right)$, the inequality

$$
|\langle Q u, u\rangle| \leq \epsilon|| \nabla u\left\|_{L^{2}\left(\mathbb{R}^{n}\right)}^{2}+c \epsilon^{-\nu}\right\| u \|_{L^{1}\left(\mathbb{R}^{n}\right)}^{2}, \quad \forall u \in C_{0}^{\infty}\left(\mathbb{R}^{n}\right) .
$$

(iii) There exist $\delta_{0}>0$ and $C>0$ such that, for every $\delta \in\left(0, \delta_{0}\right)$,

$$
|\langle Q u, u\rangle| \leq C \delta^{\frac{2}{\beta+1}}\|\nabla u\|_{L^{2}\left(\mathbb{R}^{n}\right)}^{2}, \quad \forall u \in C_{0}^{\infty}\left(B_{\delta}\left(x_{0}\right)\right),
$$

where $C$ does not depend on $x_{0}$ and $\delta$. 
Moreover, in statements (i)-(iii) one may set $\epsilon_{0}=1$ if $\delta_{0}=1$ and vice versa. Similarly, if $\epsilon_{0}=+\infty$ in (i) or (ii) then $\delta_{0}=+\infty$ in (iii), and the converse is also true.

Proof. Let $\alpha=\frac{n}{n+2}$. Applying Nash's inequality ([LL, Theorem 2.13),

$$
\|\nabla u\|_{L^{2}\left(\mathbb{R}^{n}\right)} \leq C(n)\|\nabla u\|_{L^{2}\left(\mathbb{R}^{n}\right)}^{\alpha}\|u\|_{L^{1}\left(\mathbb{R}^{n}\right)}^{1-\alpha}, \quad \forall u \in C_{0}^{\infty}\left(\mathbb{R}^{n}\right),
$$

we see that (3.9) yields

$$
|\langle Q u, u\rangle| \leq C \epsilon\|\nabla u\|_{L^{2}\left(\mathbb{R}^{n}\right)}^{2}+c \epsilon^{-\beta}\|\nabla u\|_{L^{2}\left(\mathbb{R}^{n}\right)}^{2 \alpha}\|u\|_{L^{1}\left(\mathbb{R}^{n}\right)}^{2(1-\alpha)} .
$$

From this by Young's inequality it follows

$$
|\langle Q u, u\rangle| \leq 2 \epsilon\|\nabla u\|_{L^{2}\left(\mathbb{R}^{n}\right)}^{2}+c_{1} \epsilon^{-\nu}\|\nabla u\|_{L^{2}\left(\mathbb{R}^{n}\right)}^{2 \alpha}\|u\|_{L^{1}\left(\mathbb{R}^{n}\right)}^{2} .
$$

This proves $(\mathrm{i}) \Rightarrow(\mathrm{ii})$.

To show that (ii) $\Rightarrow\left(\right.$ iii), suppose $u \in C^{\infty}\left(B_{\delta}\left(x_{0}\right)\right)$. Then by Schwartz's inequality and (3.3),

$$
\begin{aligned}
|\langle Q u, u\rangle| & \leq \epsilon\|u\|_{L^{1}\left(B_{\delta}\left(x_{0}\right)\right)}^{2}+c \epsilon^{-\nu} \delta^{n}\|u\|_{L^{2}\left(B_{\delta}\left(x_{0}\right)\right)}^{2} \\
& \leq\left(\epsilon+c_{1} \epsilon^{-\nu} \delta^{n+2}\right)\|\nabla u\|_{L^{2}\left(B_{\delta}\left(x_{0}\right)\right)}^{2} .
\end{aligned}
$$

Minimizing over $\epsilon \in\left(0, \epsilon_{0}\right)$, we get (iii). The implication (iii) $\Rightarrow$ (i) is a direct consequence of Lemma 3.1 .

Remark 3.1. Corollary 3.3 covers the case $\nu>\frac{n}{2}$ in (3.10), since $\nu=\frac{n+2}{2} \beta+\frac{n}{2}$, where $\beta>0$. However, it is easy to see that the validity of (3.10) implies $Q=0$ if $\nu<\frac{n}{2}$, and $Q \in L^{\infty}\left(\mathbb{R}^{n}\right)$ if $\nu=\frac{n}{2}$. In the latter case, $Q \in L^{\infty}\left(\mathbb{R}^{n}\right)$ is also sufficient for (3.10) by Nash's inequality.

Indeed, suppose that (3.10) holds for $0<\nu \leq \frac{n}{2}$. Then the same inequality holds for $Q_{t}=Q \star \phi_{t}$ where $\phi_{t}(x)=t^{-n} \phi\left(t^{-1} x\right)(t>0)$ is a standard mollifier. Now using $Q_{t}$ in place of $Q$, letting $u=\eta_{\delta, x_{0}}$ in (3.10), where $\eta_{\delta, x_{0}}$ is a smooth cut-off function as in the proof of Lemma 3.1] and minimizing over $\epsilon>0$, we deduce that $\delta^{-n+2-\frac{n+2}{\nu+1}}\left|\left\langle Q_{t}, \eta_{\delta, x_{0}}^{2}\right\rangle\right| \leq C$. Letting $\delta \rightarrow 0$, we conclude that $Q_{t}\left(x_{0}\right)=0$ if $\nu<\frac{n}{2}$, and $\sup _{t>0}\left|Q_{t}\left(x_{0}\right)\right| \leq C$ if $\nu=\frac{n}{2}$, for every $x_{0} \in R^{n}$. Hence $Q=0$ if $\nu<\frac{n}{2}$, and $Q \in L^{\infty}\left(\mathbb{R}^{n}\right)$ if $\nu=\frac{n}{2}$.

\section{Nonnegative potentials}

In this section, we give necessary and sufficient conditions for the infinitesimal form boundedness for nonnegative potentials $Q \in D^{\prime}\left(\mathbb{R}^{n}\right)$, i.e., nonnegative measures. The general case of distributional potentials will be considered in the next section.

Let $M^{+}\left(\mathbb{R}^{n}\right)$ denote the class of nonnegative locally finite Borel measures on $\mathbb{R}^{n}$. For a compact set $e \subset \mathbb{R}^{n}$, the capacity cap $(e)$ associated with the 
Sobolev space $W^{1,2}\left(\mathbb{R}^{n}\right)$ is defined by:

$$
\operatorname{cap}(e)=\inf \left\{\int_{\mathbb{R}^{n}}\left(|\nabla u|^{2}+|u|^{2}\right) d x: \quad u \in C_{0}^{\infty}\left(\mathbb{R}^{n}\right), u(x)>1 \text { on } e\right\} .
$$

On compact sets, cap $(\cdot)$ coincides with the so-called Bessel capacity defined by (see, e.g., $[\mathrm{AH}]$ ):

$$
\operatorname{cap}(e)=\inf \left\{\int_{\mathbb{R}^{n}} f(x)^{2} d x: \quad f \in L^{2}\left(\mathbb{R}^{n}\right), \quad f \geq 0, \quad G_{1} \star f(x) \geq 1 \text { on } e\right\},
$$

where $G_{1} \star f=(1-\Delta)^{-\frac{1}{2}} f$ is the Bessel potential of order 1 .

Theorem 4.1. Suppose $\mu \in M^{+}\left(\mathbb{R}^{n}\right), n \geq 2$. Then the following statements are equivalent.

(i) There exists $\epsilon_{0}>0$ and a function $C(\epsilon):\left(0, \epsilon_{0}\right) \rightarrow \mathbb{R}_{+}$such that the inequality

$$
\int_{\mathbb{R}^{n}}|u(x)|^{2} d \mu \leq \epsilon\|\nabla u\|_{L^{2}}^{2}+C(\epsilon)\|u\|_{L^{2}}^{2}, \quad \forall u \in C_{0}^{\infty}\left(\mathbb{R}^{n}\right),
$$

holds for every $\epsilon \in\left(0, \epsilon_{0}\right)$.

(ii)

$$
\lim _{\delta \rightarrow+0} \sup _{x_{0} \in \mathbb{R}^{n}} \sup \left\{\int_{\mathbb{R}^{n}}|u(x)|^{2} d \mu: u \in C_{0}^{\infty}\left(B_{\delta}\left(x_{0}\right)\right),\|\nabla u\|_{L^{2}} \leq 1\right\}=0 .
$$

$$
\lim _{\delta \rightarrow+0} \sup \left\{\frac{\mu(e)}{\operatorname{cap}(e)}: \quad e \subset \mathbb{R}^{n}, \operatorname{diam}(e) \leq \delta\right\}=0,
$$

where the supremum above is over compact sets e of positive capacity.

$$
\lim _{\delta \rightarrow+0} \sup \left\{\frac{1}{\mu\left(P_{0}\right)} \sum_{P \subseteq P_{0}}|P|^{\frac{2}{n}-1} \mu(P)^{2}: \quad P_{0} \subset \mathbb{R}^{n}, \operatorname{diam}\left(P_{0}\right) \leq \delta\right\}=0,
$$

where the supremum is over dyadic cubes $P_{0}$ such that $\mu\left(P_{0}\right) \neq 0$, and the sum is over all dyadic cubes $P$ contained in $P_{0}$.

Obviously, in statement (i) one can assume that $C(\epsilon)$ is defined on $\mathbb{R}^{+}=$ $(0,+\infty)$ so that (4.2) holds for every $\epsilon>0$.

Proof. By Lemma 3.1, (i) $\Leftrightarrow$ (ii). We next show that (ii) $\Leftrightarrow$ (iii).

Let $\Omega \subset \mathbb{R}^{n}$ be an open set, and let $e$ be a compact subset of $\Omega$. We will need the the Wiener capacity relative to the domain $\Omega$ defined by:

$$
\operatorname{cap}(e, \Omega)=\inf \left\{\int_{\Omega}|\nabla u|^{2} d x: \quad u \in C_{0}^{\infty}(\Omega), u>1 \text { on } e\right\} .
$$


We remark that, for $\Omega=\mathbb{R}^{n}$, in the case $n \geq 3$ and $\operatorname{diam}(e) \leq 1$, we have:

$$
\operatorname{cap}\left(e, \mathbb{R}^{n}\right) \leq \operatorname{cap}(e) \leq c(n) \operatorname{cap}\left(e, \mathbb{R}^{n}\right),
$$

where $c(n)$ depends only on $n$. The left-hand side estimate is obvious, and the right-hand side follows, e.g., from Hardy's inequality. For $n=1,2$ it is easy to see that cap $\left(e, \mathbb{R}^{n}\right)=0$ for every $e \subset \mathbb{R}^{n}$ (see [M2]).

We now set

$$
c_{1}(\mu, \Omega)=\sup \left\{\int_{\mathbb{R}^{n}}|u(x)|^{2} d \mu: u \in C_{0}^{\infty}(\Omega),\|\nabla u\|_{L^{2}(\Omega)} \leq 1\right\}
$$

and

$$
c_{2}(\mu, \Omega)=\sup \left\{\frac{\mu(e)}{\operatorname{cap}(e, \Omega)}: \quad e \subset \Omega\right\},
$$

where the supremum above is over compact sets $e \subset \Omega$ of positive capacity. As was shown in [M1] (see also [M2], Sec. 2.5),

$$
c_{2}(\mu, \Omega) \leq c_{1}(\mu, \Omega) \leq 4 c_{2}(\mu, \Omega) .
$$

Applying this inequality with $\Omega=B_{\delta}\left(x_{0}\right)$, we see that (4.3) holds if and only if

$$
\lim _{\delta \rightarrow+0} \sup _{x_{0} \in \mathbb{R}^{n}} \sup \left\{\frac{\mu(e)}{\operatorname{cap}\left(e, B_{\delta}\left(x_{0}\right)\right)}: \quad e \subset B_{\delta}\left(x_{0}\right)\right\}=0,
$$

where $e$ is a compact subset of $B_{\delta}\left(x_{0}\right)$.

To verify (ii) $\Leftrightarrow($ iii), it remains to prove that one can replace the capacity cap $\left(e, B_{\delta}\left(x_{0}\right)\right)$ in (4.11) with cap $(e)$ where $e$ is a compact set such that $\operatorname{diam}(e) \leq \delta$. Without loss of generality we may assume that $0<\delta \leq 1$.

Notice that if $e \subset B_{\delta}\left(x_{0}\right)$ and $\delta \leq 1$, then, for every $u \in C_{0}^{\infty}\left(B_{\delta}\left(x_{0}\right)\right)$,

$$
\int_{\mathbb{R}^{n}}\left(|\nabla u|^{2}+|u|^{2}\right) d x \leq \int_{\mathbb{R}^{n}}\left(|\nabla u|^{2}+\delta^{-2}|u|^{2}\right) d x \leq c(n) \int_{\mathbb{R}^{n}}|\nabla u|^{2} d x,
$$

by (3.3). Hence, minimizing both sides over $u$ such that $u(x)>1$ on $e$, we get:

$$
\operatorname{cap}(e) \leq c(n) \operatorname{cap}\left(e, B_{\delta}\left(x_{0}\right)\right) .
$$

This estimate, which holds for every $n \geq 1$, yields (iii) $\Rightarrow$ (ii).

In the opposite direction, suppose first that $n \geq 3$. Then we will show that

$$
\operatorname{cap}\left(e, B_{\widetilde{\delta}}\left(x_{0}\right)\right) \leq c \operatorname{cap}(e),
$$

for every $e \subset B_{\delta}\left(x_{0}\right)$, where $\widetilde{\delta}=2 \delta$, and $c$ depends only on $n$.

Indeed, suppose $u \in C_{0}^{\infty}\left(\mathbb{R}^{n}\right)$, where $u(x)>1$ on $e$, and

$$
\int_{\mathbb{R}^{n}}\left(|\nabla u|^{2}+|u|^{2}\right) d x \leq 2 \operatorname{cap}(e) .
$$


Denote by $\eta_{2 \delta, x_{0}}(x)=\eta\left(\frac{x-x_{0}}{2 \delta}\right)$ a smooth cut-off function supported in $B_{2 \delta\left(x_{0}\right)}$ so that $\eta_{2 \delta, x_{0}}(x)=1$ on $B_{\delta}\left(x_{0}\right)$. Then $v(x)=\eta_{2 \delta, x_{0}}(x) u(x) \in C_{0}^{\infty}\left(B_{2 \delta}\left(x_{0}\right)\right)$, and $v(x)>1$ on $e$. We estimate using Hardy's inequality:

$$
\begin{aligned}
\int_{B_{2 \delta}\left(x_{0}\right)}|\nabla v|^{2} d x & \leq 2 \int_{B_{2 \delta}\left(x_{0}\right)}\left(\left|\eta_{2 \delta, x_{0}}\right|^{2}|\nabla u|^{2}+\left|\nabla \eta_{2 \delta, x_{0}}\right|^{2}|u|^{2}\right) d x \\
& \leq c \int_{B_{2 \delta}\left(x_{0}\right)}\left(|\nabla u|^{2}+\delta^{-2}|u|^{2}\right) d x \leq c_{1} \int_{\mathbb{R}^{n}}|\nabla u|^{2} d x
\end{aligned}
$$

where $c, c_{1}$ depend only on $n$. Thus,

$$
\operatorname{cap}\left(e, B_{2 \delta}\left(x_{0}\right)\right) \leq \int_{B_{2 \delta}\left(x_{0}\right)}|\nabla v|^{2} d x \leq c_{1} \int_{\mathbb{R}^{n}}|\nabla u|^{2} d x \leq 2 c_{1} \operatorname{cap}(e),
$$

which proves (4.12) with $\widetilde{\delta}=2 \delta$. From this it is immediate that (ii) $\Rightarrow$ (iii) if $n \geq 3$.

In the case $n=2$ we use a more delicate argument with $\widetilde{\delta} \in(\delta, 1)$ to be determined later. We denote by $\nu=\nu_{e} \in M^{+}\left(\mathbb{R}^{2}\right)$ the equilibrium measure associated with a compact set $e \subset \mathbb{R}^{2}$ such that (see [AH], M2]):

$$
\nu(e)=\operatorname{cap}(e)=c(n) \int_{\mathbb{R}^{2}}\left(|\nabla \mathcal{P} \nu|^{2}+(\mathcal{P} \nu)^{2} \mid\right) d x, \quad \operatorname{supp}(\nu) \subset e .
$$

Here $\mathcal{P} \nu=(1-\Delta)^{-1} \nu=G_{2} \star \nu$ is the Bessel potential of order 2 :

$$
\mathcal{P} \nu(x)=\int_{\mathbb{R}^{2}} G_{2}(x-y) d \nu(y),
$$

where the Bessel kernel $G_{\alpha}$ of order $\alpha$ on $\mathbb{R}^{n}$ is defined through the Fourier transform:

$$
\widehat{G}_{\alpha}(\xi)=\left(1+|\xi|^{2}\right)^{-1}, \quad \xi \in \mathbb{R}^{n}, \quad \alpha>0 .
$$

Without loss of generality we may assume $\mathcal{P} \nu \in C^{\infty}\left(\mathbb{R}^{2}\right)$. (This assumption, which is not essential to the proof, is easily removed by using an appropriate mollifier $\mathcal{P} \nu \star \zeta_{r}$, where $\zeta_{r}(x)=r^{-2} \zeta\left(\frac{x}{r}\right)$, and letting $r \rightarrow+\infty$.)

Let $v(x)=\eta_{\tilde{\delta}}(x) \mathcal{P} \nu(x) \in C_{0}^{\infty}\left(B_{\widetilde{\delta}}\left(x_{0}\right)\right)$. It follows that

$$
\operatorname{cap}\left(e, B_{\widetilde{\delta}}\left(x_{0}\right)\right) \leq \int_{B_{\tilde{\delta}}\left(x_{0}\right)}|\nabla v|^{2} d x \leq \int_{B_{\tilde{\delta}}\left(x_{0}\right)}|\nabla \mathcal{P} \nu|^{2} d x+c \widetilde{\delta}^{-2} \int_{B_{\tilde{\delta}}\left(x_{0}\right)}(\mathcal{P} \nu)^{2} d x
$$

The first term on the right is bounded by cap $(e)$. We rewrite the integral in the second term in the form (note that $\nu$ is supported on $e$ ):

$$
\int_{B_{\tilde{\delta}}\left(x_{0}\right)}(\mathcal{P} \nu)^{2} d x=\int_{e} \int_{e} \int_{B_{\tilde{\delta}}\left(x_{0}\right)} G_{2}(x-y) G_{2}\left(x-y^{\prime}\right) d x d \nu(y) d \nu\left(y^{\prime}\right),
$$


and estimate using the fact that $G_{2}(x) \leq c \log \frac{4}{|x|}$ for $|x| \leq 2$ in the case $n=2$ (see, e.g., $\left[\mathrm{AH}\right.$, Sec. 1.2.5). Since $e \subset B_{\delta}\left(x_{0}\right)$ and $\max \left(|x-y|,\left|x-y^{\prime}\right|\right)<2 \widetilde{\delta}$, we have:

$$
\begin{aligned}
& \int_{B_{\tilde{\delta}}\left(x_{0}\right)} G_{2}(x-y) G_{2}\left(x-y^{\prime}\right) d x \leq \int_{B_{\widetilde{\delta}}\left(x_{0}\right)}\left(G_{2}(x-y)^{2}+G_{2}\left(x-y^{\prime}\right)^{2}\right) d x \\
& \leq c \int_{|x-y|<2 \widetilde{\delta}} \log ^{2} \frac{4}{|x-y|} d x+c \int_{\left|x-y^{\prime}\right|<2 \widetilde{\delta}} \log ^{2} \frac{4}{\left|x-y^{\prime}\right|} d y^{\prime} \leq c \widetilde{\delta}^{2} \log ^{2} \frac{2}{\widetilde{\delta}} .
\end{aligned}
$$

It follows:

$$
\int_{B_{\tilde{\delta}}\left(x_{0}\right)}(\mathcal{P} \nu)^{2} d x \leq c \nu(e)^{2} \widetilde{\delta}^{2} \log ^{2} \frac{2}{\widetilde{\delta}}=c \operatorname{cap}(e)^{2} \widetilde{\delta}^{2} \log ^{2} \frac{2}{\widetilde{\delta}} .
$$

Hence,

$$
\operatorname{cap}\left(e, B_{\widetilde{\delta}}\left(x_{0}\right)\right) \leq c\left(\operatorname{cap}(e)+\log ^{2} \underset{\widetilde{\delta}}{\frac{2}{\widetilde{\delta}}} \operatorname{cap}(e)^{2}\right),
$$

where $e \subset B_{\delta}\left(x_{0}\right)$ and $c$ depends only on $n$. Using the known estimate of the capacity of a ball ([M2], Sec. 7.2.3):

$$
\operatorname{cap}\left(B_{\delta}\left(x_{0}\right)\right) \asymp \frac{1}{\log \frac{2}{\delta}}, \quad 0<\delta<1, \quad n=2,
$$

we have:

$$
\operatorname{cap}(e) \leq \operatorname{cap}\left(B_{\delta}\left(x_{0}\right)\right) \leq \frac{c}{\log \frac{2}{\delta}} .
$$

Combining the preceding estimate and (4.16) gives:

$$
\operatorname{cap}\left(e, B_{\widetilde{\delta}}\left(x_{0}\right)\right) \leq c \operatorname{cap}(e)\left(1+\frac{\log ^{2} \frac{2}{\widetilde{\delta}}}{\log \frac{2}{\delta}}\right), \quad e \subset B_{\delta}\left(x_{0}\right), \quad n=2 .
$$

Now choosing $\widetilde{\delta}$ so that $\log ^{2} \frac{2}{\tilde{\delta}}=\log \frac{2}{\delta}$, we get (4.12). Letting $\delta \rightarrow+0$, so that $\widetilde{\delta} \rightarrow+0$, we obtain that (ii) $\Rightarrow$ (iii) in the case $n=2$. This proves (ii) $\Leftrightarrow$ (iii).

To prove (iii) $\Leftrightarrow($ iv), we set

$$
\kappa_{1}(\mu, \delta)=\sup \left\{\frac{\mu(e)}{\operatorname{cap}(e)}: \quad \operatorname{diam}(e) \leq \delta\right\},
$$

where $e$ is a compact set of positive capacity in $\mathbb{R}^{n}$, and

$$
\kappa_{2}(\mu, \delta)=\sup \left\{\frac{1}{\mu\left(P_{0}\right)} \sum_{P \subseteq P_{0}}|P|^{\frac{2}{n}-1} \mu(P)^{2}: \quad \operatorname{diam}\left(P_{0}\right) \leq \delta\right\},
$$

where the supremum is over dyadic cubes $P_{0}$ in $\mathbb{R}^{n}$ such that $\mu\left(P_{0}\right) \neq 0$. 
For a set $e \subset \mathbb{R}^{n}$, denote by $\mu_{e}$ the restriction of the measure $\mu$ to $e$, i.e., $d \mu_{e}=\chi_{e} d \mu$. Denote by

$$
\mathcal{E}(\mu)=\|\mu\|_{W^{-1,2}\left(\mathbb{R}^{n}\right)}^{2}=\int_{\mathbb{R}^{n}}\left(G_{1} \star \mu\right)^{2} d x
$$

the energy of the measure $\mu$.

We first show that, for any compact set $e$ such that $\operatorname{diam}(e) \leq \delta$,

$$
\mathcal{E}\left(\mu_{e}\right) \leq c \kappa_{1}(\mu, \delta) \mu(e) .
$$

Such inequalities, without the restriction on the diameter of $e$, are known (see, e.g., [M2], [V2] ) and we will be brief here. Clearly, there exists $g \in C_{0}^{\infty}\left(\mathbb{R}^{n}\right)$, $g \geq 0$, such that $\|g\|_{L^{2}\left(\mathbb{R}^{n}\right)}=1$, and

$$
\mathcal{E}\left(\mu_{e}\right)^{\frac{1}{2}} \leq 2 \int_{\mathbb{R}^{n}} G_{1} \star \mu_{e} g d x=2 \int_{e} G_{1} \star g d \mu .
$$

For any $\lambda>0$, set $e_{\lambda}=\left\{x: G_{1} \star g(x)>\lambda\right\}$. Then

$$
\mu_{e}\left(e_{\lambda}\right)=\mu\left(e_{\lambda} \cap e\right) \leq \kappa_{1}(\mu, \delta) \operatorname{cap}\left(e_{\lambda} \cap e\right) \leq c \kappa_{1}(\mu, \delta)\left\|\frac{g}{\lambda}\right\|_{L^{2}\left(\mathbb{R}^{n}\right)}^{2},
$$

where the last estimate follows from the definition of the capacity since

$$
u(x)=\left(G_{1} \star \frac{g}{\lambda}\right)(x)>1, \quad x \in e_{\lambda},
$$

and $\|u\|_{W^{1,2}\left(\mathbb{R}^{n}\right)}^{2}=c\|g\|_{L^{2}\left(\mathbb{R}^{n}\right)}^{2}=c$, where $c$ depend only on $n$.

In other words, $G_{1} \star g \in L^{2, \infty}\left(\mu_{e}\right)$, and

$$
\left\|G_{1} \star g\right\|_{L^{2, \infty}\left(\mu_{e}\right)} \leq c \kappa_{1}(\mu, \delta)^{\frac{1}{2}}
$$

where $L^{2, \infty}(\nu)$ denotes the corresponding Lorentz (weak $L^{2}$ ) space on $\mathbb{R}^{n}$ equipped with the measure $\nu$. Hence,

$$
\int_{e} G_{1} \star g d \mu \leq c\left\|G_{1} \star g\right\|_{L^{2, \infty}\left(\mu_{e}\right)} \mu(e)^{\frac{1}{2}} \leq c \kappa_{1}(\mu, \delta)^{\frac{1}{2}} \mu(e)^{\frac{1}{2}},
$$

which proves (4.21).

Letting $e=P_{0}$ in (4.21) where $P_{0}$ is a dyadic cube such that diam $\left(P_{0}\right) \leq \delta$, we get

$$
\mathcal{E}\left(\mu_{P_{0}}\right) \leq \kappa_{1}(\mu, \delta) \mu\left(P_{0}\right) .
$$

For $x, y \in P_{0}$, we have $|x-y| \leq \delta \leq 1$, and it is easy to see that

$$
\int_{P_{0}} G_{1}(x-y) d \mu(y) \geq \int_{P_{0}} \frac{d \mu(y)}{|x-y|^{n-1}} \geq c \sum_{P \subseteq P_{0}}|P|^{\frac{1}{n}-1} \mu(P) \chi_{P}(x) .
$$

We estimate:

$$
\mathcal{E}\left(\mu_{P_{0}}\right) \geq c \int_{P_{0}}\left(G_{1} \star \mu_{P_{0}}\right)^{2} d x \geq c \int_{P_{0}}\left(\sum_{P \subseteq P_{0}}|P|^{\frac{1}{n}-1} \mu(P) \chi_{P}(x)\right)^{2} d x
$$




$$
\geq \int_{P_{0}} \sum_{P \subseteq P_{0}}|P|^{\frac{2}{n}-2} \mu(P)^{2} \chi_{P}(x) d x=\sum_{P \subseteq P_{0}}|P|^{\frac{2}{n}-1} \mu(P)^{2} .
$$

Thus,

$$
\sum_{P \subseteq P_{0}}|P|^{\frac{2}{n}-1} \mu(P)^{2} \leq c \kappa_{1}(\mu, \delta) \mu\left(P_{0}\right)
$$

that is, $\kappa_{2}(\mu, \delta) \leq c \kappa_{1}(\mu, \delta)$. This estimate yields (iii) $\Rightarrow($ iv).

The converse can be proved as in V2 in the case of Riesz kernels using Th. Wolff's inequality [HW]. (See also COV] where Wolff's inequality is proved for general dyadic and radial kernels.) Here we sketch a direct proof based on the dyadic Carleson measure theorem (see, e.g., V1]) which yields the inequality

$$
\sum_{P \subseteq P_{0}}|P|^{\frac{2}{n}-1} \mu(P)^{2}\left(\frac{1}{\mu(P)} \int_{P} g d \mu\right)^{2} \leq c \kappa_{2}(\mu, \delta) \int_{P_{0}} g^{2} d \mu
$$

where $g \in L^{2}\left(\mu_{P_{0}}\right), g \geq 0$, for every dyadic cube $P_{0}$ such that $\operatorname{diam}\left(P_{0}\right) \leq \delta$. (This inequality follows by interpolation between the trivial $L^{\infty}\left(\mu_{P_{0}}\right) \rightarrow l^{\infty}$ estimate and the weak-type $(1,1)$ estimate from $L^{1}\left(\mu_{P_{0}}\right)$ to $l^{1, \infty}$. Note that the left-hand side of the preceding inequality is bounded from below by

$$
\int_{P_{0}}\left(\sum_{P \subseteq P_{0}}|P|^{\frac{1}{n}-1} \chi_{P}(x) \int_{P} g d \mu\right)^{2} d x .
$$

To verify this estimate, which is closely related to Wolff's inequality, we use the obvious pointwise inequality:

$$
\begin{aligned}
& \left(\sum_{P \subseteq P_{0}}|P|^{\frac{1}{n}-1} \chi_{P}(x) \int_{P} g d \mu\right)^{2} \\
& \leq 2 \sum_{P \subseteq P_{0}}|P|^{\frac{1}{n}-1}\left(\int_{P} g d \mu\right) \sum_{P^{\prime} \subseteq P}\left|P^{\prime}\right|^{\frac{1}{n}-1} \chi_{P^{\prime}}(x)\left(\int_{P^{\prime}} g d \mu\right) .
\end{aligned}
$$

Integrating both sides of the preceding inequality over $P_{0}$, and the estimate

$$
\sum_{P^{\prime} \subseteq P}\left|P^{\prime}\right|^{\frac{1}{n}}\left(\int_{P^{\prime}} g d \mu\right) \leq c(n)|P|^{\frac{1}{n}} \int_{P} g d \mu
$$

we obtain

$$
\begin{aligned}
\int_{P_{0}}\left(\sum_{P \subseteq P_{0}}|P|^{\frac{1}{n}-1} \chi_{P}(x) \int_{P} g d \mu\right)^{2} d x & \leq c \sum_{P \subseteq P_{0}}|P|^{\frac{2}{n}-1}\left(\int_{P} g d \mu\right)^{2} \\
& \leq c \kappa_{2}(\mu, \delta) \int_{P_{0}} g^{2} d \mu
\end{aligned}
$$


By duality, this gives

$$
\int_{P_{0}}\left(\sum_{P \subseteq P_{0}}|P|^{\frac{1}{n}-1} \chi_{P}(x) \int_{P} f(y) d y\right)^{2} d \mu(x) \leq c \kappa_{2}(\mu, \delta) \int_{P_{0}} f(y)^{2} d y
$$

for every $f \in L^{2}(d x), f \geq 0$. Note that

$$
I_{1}^{P_{0}} f(x)=\sum_{P \subseteq P_{0}}|P|^{\frac{1}{n}-1} \chi_{P}(x) \int_{P} f(y) d y
$$

is the dyadic Riesz potential of order 1 (see [HW] scaled to the cube $P_{0}$. It is easy to see that this estimate, which holds for every dyadic cube $P_{0}$ such that $\operatorname{diam}\left(P_{0}\right) \leq \delta \leq 1$, implies the inequality

$$
\int_{P_{0}}\left(G_{1} \star f\right)^{2} d \mu \leq c \kappa_{2}(\mu, \delta)\|f\|_{L^{2}(d x)}^{2},
$$

for every $f \in L^{2}(d x)$. (See details in $[\mathrm{V} 2$ or $\mathrm{COV}$.)

From this and the definition of the Bessel capacity it is immediate that

$$
\mu(e) \leq c \kappa_{2}(\mu, \delta) \operatorname{cap}(e), \quad \operatorname{diam}(e) \leq \delta .
$$

Hence, $\kappa_{1}(\mu, \delta) \leq c \kappa_{2}(\mu, \delta)$, where $c$ depends only on $n$. From this it follows that (iv) $\Rightarrow$ (iii). This completes the proof of Theorem 4.1 .

Corollary 4.2. Let $\mu \in M^{+}\left(\mathbb{R}^{n}\right)$. If inequality (4.2) holds then

$$
\begin{aligned}
& \lim _{\delta \rightarrow+0} \sup \left\{\frac{\mu\left(B_{\delta}\left(x_{0}\right)\right)}{\delta^{n-2}}: \quad x_{0} \in \mathbb{R}^{n}\right\}=0, \quad n \geq 3, \\
& \lim _{\delta \rightarrow+0} \sup \left\{\log \frac{1}{\delta} \mu\left(B_{\delta}\left(x_{0}\right)\right): \quad x_{0} \in \mathbb{R}^{n}\right\}=0, \quad n=2 .
\end{aligned}
$$

Corollary 3.2 follows by letting $e=B_{\delta}\left(x_{0}\right)$ in Theorem 4.1 (iii), and using the estimates cap $\left(B_{\delta}\left(x_{0}\right)\right) \asymp \delta^{n-2}$ if $n \geq 3$ and cap $\left(B_{\delta}\left(x_{0}\right)\right) \asymp \frac{1}{\log \frac{2}{\delta}}$ if $n=2$, provided $0<\delta \leq 1$ ( see [M2]).

Stronger necessary conditions are obtained by replacing $\mu\left(B_{\delta}\left(x_{0}\right)\right)$ above with $\int_{B_{\delta}\left(x_{0}\right)}\left(G_{1} \star \mu\right)^{2} d x$.

Corollary 4.3. Let $\mu \in M^{+}\left(\mathbb{R}^{n}\right)$. If inequality (4.2) holds then

$$
\begin{aligned}
& \lim _{\delta \rightarrow+0} \sup \left\{\frac{\int_{B_{\delta}\left(x_{0}\right)}\left(G_{1} \star \mu\right)^{2} d x}{\delta^{n-2}}: \quad x_{0} \in \mathbb{R}^{n}\right\}=0, \quad n \geq 3, \\
& \lim _{\delta \rightarrow+0} \sup \left\{\log \frac{1}{\delta} \int_{B_{\delta}\left(x_{0}\right)}\left(G_{1} \star \mu\right)^{2} d x: \quad x_{0} \in \mathbb{R}^{n}\right\}=0, \quad n=2 .
\end{aligned}
$$


This corollary is a consequence of the results of [MV2 ( see also Sec. [5] below).

Various related results can be deduced from Theorem 4.1 using known form boundedness criteria (see, e.g., [Fef], [ChWW], [KS], [M2], [MV1, [MV2], V2]). In particular, we can give more equivalent conditions which are necessary and sufficient for (4.2).

Corollary 4.4. Let $\mu \in M^{+}\left(\mathbb{R}^{n}\right)$. Then inequality (4.2) is equivalent to any one of the following conditions:

(i)

$$
\lim _{\delta \rightarrow+0} \sup \left\{\frac{\mathcal{E}\left(\mu_{B_{\delta}\left(x_{0}\right)}\right)}{\mu\left(B_{\delta}\left(x_{0}\right)\right)}: \quad x_{0} \in \mathbb{R}^{n}\right\}=0
$$

$$
\lim _{\delta \rightarrow+0} \sup \left\{\frac{\left\|\chi_{B_{\delta}\left(x_{0}\right)} \mu\right\|_{W^{-1,2}\left(\mathbb{R}^{n}\right)}^{2}}{\mu\left(B_{\delta}\left(x_{0}\right)\right)}: \quad x_{0} \in \mathbb{R}^{n}\right\}=0 .
$$

$$
\lim _{\delta \rightarrow+0} \sup \left\{\frac{G_{1} \star\left(G_{1} \star \mu_{B_{\delta}\left(x_{0}\right)}\right)^{2}(x)}{G_{1} \star \mu_{B_{\delta}\left(x_{0}\right)}(x)}: \quad x, x_{0} \in \mathbb{R}^{n}\right\}=0 .
$$

Statements (i) and (ii) of Corollary 4.3 follow directly from the proof of Theorem 4.1 while (iii) can be proved in a similar way using results of [MV1].

Simpler sufficient conditions can be deduced easily from Theorem 4.1. For instance, it is well known that if $d \mu=\rho(x) d x$ where $\rho \in L^{\frac{n}{2}}\left(\mathbb{R}^{n}\right)+L^{\infty}\left(\mathbb{R}^{n}\right), n \geq$ 3 , then (4.2) holds. Moreover, it can be replaced with the "locally uniform" $L^{\frac{n}{2}}$-condition (see $[\mathrm{BrK}]$ ):

$$
\lim _{\delta \rightarrow+0} \sup _{x_{0} \in \mathbb{R}^{n}}\|\rho\|_{L^{\frac{n}{2}}\left(B_{\delta}\left(x_{0}\right)\right)}=0 .
$$

In fact, one can use here a local weak- $L^{\frac{n}{2}}$, and even a local Fefferman-Phong norm.

Corollary 4.5. Let $d \mu=\rho(x) d x$, where $\rho \in L_{\text {loc }}^{r}\left(\mathbb{R}^{n}\right)$ for some $r>1$ and $n \geq 3$. Then (4.2) holds if

$$
\lim _{\delta \rightarrow+0} \sup _{x_{0} \in \mathbb{R}^{n}} \frac{\int_{B_{\delta}\left(x_{0}\right)} \rho(x)^{r} d x}{\delta^{n-2 r}}=0 .
$$

Furthermore, this condition can be replaced with a weaker one, with $\left(G_{1} \star \mu\right)^{2}$ in place of $\rho$. 
Another well-known sufficient condition for (4.2) which is generally not covered by the preceding corollary is Kato's condition $K_{n}$ :

$$
\begin{aligned}
& \lim _{\delta \rightarrow+0} \sup _{x_{0} \in \mathbb{R}^{n}} \int_{B_{\delta}\left(x_{0}\right)} \frac{\rho(x)}{\left|x-x_{0}\right|^{n-2}} d x=0, \quad n \geq 3, \\
& \lim _{\delta \rightarrow+0} \sup _{x_{0} \in \mathbb{R}^{n}} \int_{B_{\delta}\left(x_{0}\right)} \log \frac{1}{\left|x-x_{0}\right|} \rho(x) d x=0, \quad n=2,
\end{aligned}
$$

where $\rho \geq 0$ and $\rho \in L_{\text {loc }}^{1}\left(\mathbb{R}^{n}\right)$.

Remark 4.1. This class can be broadened in the same way as above by replacing $\rho$ with $\left(G_{1} \star \mu\right)^{2}$ and using Theorem 4.1,

In the opposite direction, the following remarkable fact was proved in AiSi] using a probabilistic argument: Kato's condition $K_{n}$ follows from (4.2) provided

$$
C(\epsilon) \leq a e^{b \epsilon^{-p}}, \quad \epsilon \in\left(0, \epsilon_{0}\right), \quad 0<p<1 .
$$

Such results can also be deduced by operator semigroup methods LPS. In fact, the same conclusion has been obtained under a more general assumption in terms of the Legendre transform $\hat{C}(s)$ defined by (3.7):

$$
\int_{\delta_{0}}^{+\infty} \frac{\hat{C}(s)}{s^{2}} d s<+\infty
$$

for some $\delta_{0}>0$. (See Gr1, Gr2]; an analogous fact is now known for general elliptic operators with good heat kernel bounds [Dav1].) It is worth noting that, for a decreasing $C(\epsilon)$ such that $\lim _{\epsilon \rightarrow+0} C(\epsilon)=+\infty$, the preceding condition is equivalent to ([Ko], Sec. VII.D.2):

$$
\int_{0}^{\epsilon_{0}} \log C(\epsilon) d \epsilon<+\infty
$$

for some $\epsilon_{0}>0$.

We should emphasize that such results can be deduced directly from Corollary 3.2 in a sharper form. In particular, for $n=2$, it suffices to assume that $C(\epsilon) \leq a e^{b \epsilon^{-p}}$ for any $p>0$.

Proposition 4.6. Let $d \mu=\rho(x) d x$, where $\rho \geq 0$, and $\rho \in L_{\mathrm{loc}}^{1}\left(\mathbb{R}^{n}\right)$. Suppose that (4.2) holds with $C(\epsilon)$ obeying condition (4.30) if $n \geq 3$, or

$$
\int_{\delta_{0}}^{+\infty} \frac{\hat{C}(s)}{s^{2} \log s} d s<+\infty, \quad n=2,
$$

for some $\delta_{0}>1$. Then Kato's condition $K_{n}$ is valid. Moreover,

$$
\sup _{x_{0} \in \mathbb{R}^{n}} \int_{B_{\delta}\left(x_{0}\right)} \frac{\rho(x)}{\left|x-x_{0}\right|^{n-2}} d x \leq c \int_{\delta^{-2}}^{+\infty} \frac{\hat{C}(s)}{s^{2}} d s, \quad n \geq 3,
$$




$$
\sup _{x_{0} \in \mathbb{R}^{n}} \int_{B_{\delta}\left(x_{0}\right)} \log \frac{1}{\left|x-x_{0}\right|} \rho(x) d x \leq c \int_{\delta^{-2}}^{+\infty} \frac{\hat{C}(s)}{s^{2} \log s} d s, \quad n=2,
$$

where $c>0$ is a constant which depends only on $n$, and $\delta$ is sufficiently small.

Proof. Suppose that (4.32) holds. By Corollary 3.2.

$$
\int_{B_{\delta}\left(x_{0}\right)}|u(x)|^{2} d \mu \leq k \delta^{2} \hat{C}\left(\delta^{-2}\right)\|\nabla u\|^{2}, \quad \forall u \in C_{0}^{\infty}\left(B_{\delta}\left(x_{0}\right)\right),
$$

where $k$ depends only on $n$. Let $u>1$ on $B_{\frac{\delta}{2}}\left(x_{0}\right)$, where $u \in C_{0}^{\infty}\left(B_{\delta}\left(x_{0}\right)\right)$. Taking the infimum over all such test functions $u$ in the preceding estimate, we obtain:

$$
\mu\left(B_{\frac{\delta}{2}}\left(x_{0}\right)\right) \leq c \delta^{2} \hat{C}\left(\delta^{-2}\right) \operatorname{cap}\left(B_{\frac{\delta}{2}}\left(x_{0}\right), B_{\delta}\left(x_{0}\right)\right),
$$

where $\operatorname{cap}\left(\cdot, B_{\delta}\left(x_{0}\right)\right)$ is the capacity (4.6). Using the estimates (see [M2]):

$$
\begin{aligned}
& \operatorname{cap}\left(\mathrm{B}_{\frac{\delta}{2}}\left(\mathrm{x}_{0}\right), \mathrm{B}_{\delta}\left(\mathrm{x}_{0}\right)\right) \asymp \delta^{n-2}, \quad n \geq 3, \\
& \operatorname{cap}\left(\mathrm{B}_{\frac{\delta}{2}}\left(\mathrm{x}_{0}\right), \mathrm{B}_{\delta}\left(\mathrm{x}_{0}\right)\right) \asymp\left(\log \frac{2}{\delta}\right)^{-1}, \quad n=2,
\end{aligned}
$$

where $0<\delta<1$, together with the inequality $\hat{C}(2 s) \leq 2 \hat{C}(s)(s>0)$, we deduce:

$$
\begin{aligned}
& \mu\left(B_{\delta}\left(x_{0}\right)\right) \leq c \delta^{n} \hat{C}\left(\delta^{-2}\right), \quad n \geq 3, \\
& \mu\left(B_{\delta}\left(x_{0}\right)\right) \leq c \delta^{2} \hat{C}\left(\delta^{-2}\right)\left(\log \frac{2}{\delta}\right)^{-1}, \quad n=2 .
\end{aligned}
$$

Suppose $d \mu=\rho(x) d x$ and $n \geq 3$. We estimate:

$$
\begin{aligned}
\int_{B_{\delta}\left(x_{0}\right)} \frac{\rho(x)}{\left|x-x_{0}\right|^{n-2}} d x & \leq c \int_{0}^{\delta} \frac{\mu\left(B_{s}\left(x_{0}\right)\right)}{s^{n-2}} \frac{d s}{s} \\
& \leq c \int_{0}^{\delta} s \hat{C}\left(s^{-2}\right) d s .
\end{aligned}
$$

In the case $n=2$ it follows:

$$
\begin{aligned}
\int_{B_{\delta}\left(x_{0}\right)} \log \frac{1}{\left|x-x_{0}\right|} \rho(x) d x & \leq c \int_{0}^{\delta} \mu\left(B_{s}\left(x_{0}\right)\right) \frac{d s}{s}+c \frac{\mu\left(B_{\delta}\left(x_{0}\right)\right)}{\delta} \\
& \leq c_{1} \int_{0}^{\delta} \frac{s \hat{C}\left(s^{-2}\right)}{\log s^{-1}} d s .
\end{aligned}
$$

Obviously, these estimates yield (4.33) and (4.34).

We conclude this section with a characterization of the subordination inequality for nonnegative measures $\mu$ :

$$
\int_{\mathbb{R}^{n}}|u(x)|^{2} d \mu \leq \epsilon\|\nabla u\|_{L^{2}\left(\mathbb{R}^{n}\right)}^{2}+\frac{C}{\epsilon^{\beta}}\|u\|_{L^{2}\left(\mathbb{R}^{n}\right)}^{2}, \quad u \in C_{0}^{\infty}\left(\mathbb{R}^{n}\right) .
$$


Theorem 4.7. Let $\mu \in M^{+}\left(\mathbb{R}^{n}\right), n \geq 2$, and let $0<\beta<+\infty$.

(i) There exists a constant $C>0$ such that (4.35) holds for every $\epsilon>0$ if and only if $\mu$ satisfies the Frostman condition:

$$
\mu\left(B_{\delta}\left(x_{0}\right)\right) \leq c \delta^{n-\frac{2 \beta}{1+\beta}},
$$

for every ball $B_{\delta}\left(x_{0}\right)$ in $\mathbb{R}^{n}$, where $c$ does not depend on $\delta>0$ and $x_{0}$.

(ii) There exists a constant $C>0$ such that (4.35) holds for every $\epsilon \in(0,1)$ if and only if (4.36) is valid for every $\delta \in(0,1)$ and $x_{0} \in \mathbb{R}^{n}$.

Statement (i) of Theorem 4.7] follows from [M2], Lemma 1.4.7, where it is proved that (4.36) holds for all $\delta>0$ if and only if the multiplicative inequality

$$
\|u\|_{L^{2}\left(\mathbb{R}^{n}, d \mu\right)} \leq C\|\nabla u\|_{L^{2}\left(\mathbb{R}^{n}, d x\right)}^{p}\|u\|_{L^{2}\left(\mathbb{R}^{n}, d x\right)}^{1-p}, \quad u \in C_{0}^{\infty}\left(\mathbb{R}^{n}\right),
$$

holds, where $p=\frac{\beta}{1+\beta} \in(0,1)$. Clearly, this inequality is equivalent to (4.35) provided it holds for every $\epsilon>0$.

There is also an inhomogeneous analogue ([M2, Corollary 1.4.7/1) which states that (4.36) holds for all $\delta \in(0,1)$ if and only if

$$
\|u\|_{L^{2}\left(\mathbb{R}^{n}, d \mu\right)} \leq C\|u\|_{W^{1,2}\left(\mathbb{R}^{n}\right)}^{p}\|u\|_{L^{2}\left(\mathbb{R}^{n}, d x\right)}^{1-p}, \quad u \in C_{0}^{\infty}\left(\mathbb{R}^{n}\right) .
$$

It is easy to see using a localization argument, as in the proof of Theorem 4.1. that this inequality holds if and only if (4.35) is valid for every $\epsilon \in(0,1)$, which yields statement (ii) of Theorem 4.7

\section{The INFINITESIMAL FORM BOUNDEDNESS CRITERION}

We are now in a position to prove Theorems I and II stated in Sec. 2. Proof of Theorem I. Suppose $Q \in \mathcal{D}^{\prime}\left(\mathbb{R}^{n}\right), n \geq 2$, is represented in the form

$$
Q=\operatorname{div} \vec{\Gamma}+\gamma, \quad \vec{\Gamma} \in \mathbf{L}_{\text {loc }}^{2}\left(\mathbb{R}^{n}\right), \quad \gamma \in L_{\text {loc }}^{1}\left(\mathbb{R}^{n}\right),
$$

where $\vec{\Gamma}$ and $\gamma$ respectively satisfy the conditions:

$$
\lim _{\delta \rightarrow+0} \sup _{x_{0} \in \mathbb{R}^{n}} \sup \left\{\||\vec{\Gamma}| u\|_{L^{2}\left(\mathbb{R}^{n}\right)}: u \in C_{0}^{\infty}\left(B_{\delta}\left(x_{0}\right)\right),\|\nabla u\|_{L^{2}\left(\mathbb{R}^{n}\right)} \leq 1\right\}=0
$$

$$
\lim _{\delta \rightarrow+0} \sup _{x_{0} \in \mathbb{R}^{n}} \sup \left\{\left\|\gamma|u|^{2}\right\|_{L^{1}\left(\mathbb{R}^{n}\right)}: u \in C_{0}^{\infty}\left(B_{\delta}\left(x_{0}\right)\right),\|\nabla u\|_{L^{2}\left(\mathbb{R}^{n}\right)} \leq 1\right\}=0 .
$$

Then, for every $\epsilon>0$, there exists $\delta>0$ so that

$$
\left\|\left(|\vec{\Gamma}|^{2}+|\gamma|\right)|u|^{2}\right\|_{L^{1}\left(\mathbb{R}^{n}\right)} \leq \epsilon\|\nabla u\|_{L^{2}\left(\mathbb{R}^{n}\right)}^{2}, \quad \forall u \in C_{0}^{\infty}\left(B_{\delta}\left(x_{0}\right)\right) .
$$

Hence by the Cauchy-Schwarz inequality,

$$
|\langle Q u, u\rangle| \leq 2|\langle\vec{\Gamma}, u \nabla u\rangle|+|\langle\gamma u, u\rangle|
$$




$$
\begin{aligned}
& \leq 2\||\vec{\Gamma}| u\|_{L^{2}\left(\mathbb{R}^{n}\right)}\|\nabla u\|_{L^{2}\left(\mathbb{R}^{n}\right)}+\left\|\gamma|u|^{2}\right\|_{L^{1}\left(\mathbb{R}^{n}\right)} \\
& \leq(2 \sqrt{\epsilon}+\epsilon)\|\nabla u\|_{L^{2}\left(\mathbb{R}^{n}\right)}^{2} .
\end{aligned}
$$

Now we take the supremum on the left-hand side over all $u \in C_{0}^{\infty}\left(B_{\delta}\left(x_{0}\right)\right)$ such that $\|\nabla u\|_{L^{2}\left(\mathbb{R}^{n}\right)}^{2} \leq 1$. Letting $\delta \rightarrow+0$, and then $\epsilon \rightarrow+0$, we obtain:

$$
\lim _{\delta \rightarrow+0} \sup _{x_{0} \in \mathbb{R}^{n}} \sup \left\{|\langle Q u, u\rangle|: u \in C_{0}^{\infty}\left(B_{\delta}\left(x_{0}\right)\right),\|\nabla u\|_{L^{2}\left(\mathbb{R}^{n}\right)} \leq 1\right\}=0 .
$$

By Lemma 3.1 this is equivalent to (3.1).

Conversely, suppose that, for every $\epsilon>0$, there exists $C(\epsilon)>0$ such that (3.1) holds. By the polarization identity, (3.1) is equivalent to

$$
\begin{aligned}
|\langle Q, u v\rangle| & \leq \epsilon^{2}\left(\|\nabla u\|_{L^{2}\left(\mathbb{R}^{n}\right)}^{2}+\frac{C(\epsilon)}{\epsilon}\|u\|_{L^{2}\left(\mathbb{R}^{n}\right)}^{2}\right)^{\frac{1}{2}} \\
& \times\left(\|\nabla v\|_{L^{2}\left(\mathbb{R}^{n}\right)}^{2}+\frac{C(\epsilon)}{\epsilon}\|v\|_{L^{2}\left(\mathbb{R}^{n}\right)}^{2}\right)^{\frac{1}{2}}, \quad \forall u, v \in C_{0}^{\infty}\left(\mathbb{R}^{n}\right) .
\end{aligned}
$$

We will show that (5.4) implies that, for every $\epsilon>0$, there exists $\delta>0$ such that

$$
\int_{e}\left(|\vec{\Gamma}(x)|^{2}+|\gamma(x)|\right) d x \leq \epsilon \operatorname{cap}(e),
$$

for every compact set $e$, diam $(e)<\delta$, where

$$
\vec{\Gamma}=-\nabla(1-\Delta)^{-1} Q, \quad \gamma=(1-\Delta)^{-1} Q,
$$

so that $Q=\operatorname{div} \vec{\Gamma}+\gamma$. Here $\operatorname{cap}(\cdot)$ is the Bessel capacity associated with the Sobolev space $W^{1,2}\left(\mathbb{R}^{n}\right)$. By Theorem 4.1, inequality (5.5) yields (5.2).

To deduce (5.5) from (5.4), we fix an arbitrary compact set $e$ of positive capacity such that diam $e \leq \delta$. Without loss of generality we may assume that $e \subset B_{\delta / 2}\left(x_{0}\right), x_{0} \in \mathbb{R}^{n}$, where $0<\delta \leq 1$. The rest of the proof makes use of the factorization method developed in MV2 combined with some new estimates for Bessel potentials of equilibrium measures.

Denote by $\mu$ the equilibrium measure associated with $e$ (see, e.g., [AH], [M2]). By $P(x)$ denote the equilibrium potential of $\mu$ defined by:

$$
P(x)=(1-\Delta)^{-1} \mu(x)=G_{2} \star \mu(x) .
$$

Here the Bessel kernel $G_{\alpha}(\cdot)$ of order $\alpha>0$ is defined by (4.15).

We will need the following well-known properties of $\mu$ and $P$ :

$$
\begin{aligned}
& \text { (a) } \operatorname{supp}(\mu) \subseteq e \\
& \text { (b) } \mu(e)=\operatorname{cap}(e) \\
& \text { (c) }\left\|(1-\Delta)^{-\frac{1}{2}} P\right\|_{L^{2}\left(\mathbb{R}^{n}\right)}^{2}=\operatorname{cap}(e)
\end{aligned}
$$




$$
\begin{aligned}
& \text { (d) } P(x)=1 \quad d \mu-\text { a.e. } \\
& \text { (e) } P(x) \leq 1 \quad \text { on } \quad \mathbb{R}^{n}
\end{aligned}
$$

We will also need the asymptotics (see [AH], Sec. 1.2.5):

$$
\begin{aligned}
& G_{2}(x) \asymp|x|^{2-n} \quad \text { if } n \geq 3, \quad G_{2}(x) \asymp \log \frac{1}{|x|} \quad \text { if } n=2, \quad|x| \rightarrow 0 ; \\
& G_{2}(x) \asymp|x|^{\frac{1-n}{2}} e^{-|x|}, \quad|x| \rightarrow+\infty, \quad n \geq 2 .
\end{aligned}
$$

Similar asymptotics hold for the derivatives of $G_{2}$ which will be used below as well.

Sometimes, it will be more convenient to use a modified kernel,

$$
\widetilde{G}_{2}(x)=\max \left(G_{2}(x), 1\right),
$$

which does not have the exponential decay at infinity. Obviously, both $G_{2}$ and $\widetilde{G}_{2}$ are positive nonincreasing radial kernels. Moreover, $\widetilde{G}_{2}$ has the doubling property:

$$
\widetilde{G}_{2}(2 x) \leq \widetilde{G}_{2}(x) \leq C(n) \widetilde{G}_{2}(2 x) .
$$

The corresponding modified potential is defined by:

$$
\widetilde{P}(x)=\widetilde{G}_{2} \star \mu(x) .
$$

Next, we fix a constant $\tau$, which ultimately will be picked in the range

$$
1<2 \tau<\min \left(\frac{n}{n-2}, 2\right), \quad n \geq 2 .
$$

The proof of statement (ii) of Theorem I is based on a series of propositions establishing some estimates for the powers of the equilibrium potential, $P(x)^{2 \tau}$.

Proposition 5.1. Let $n \geq 2$, and let $0<2 \tau<\frac{n}{n-2}$. Then $\widetilde{P}^{2 \tau}$ lies in the Muckenhoupt class $A_{1}$ on $\mathbb{R}^{n}$, i.e.,

$$
M \widetilde{P}^{2 \tau}(x) \leq C(\tau, n) \widetilde{P}^{2 \tau}(x), \quad d x \text { - a.e. }
$$

where $M$ denotes the Hardy-Littlewood maximal operator on $\mathbb{R}^{n}$, and the corresponding $A_{1}$-bound $C(\tau, n)$ depends only on $n$ and $\tau$.

Proof. Let $\rho: \mathbb{R}^{+} \rightarrow \mathbb{R}^{+}$be a nonincreasing function which satisfies the doubling condition, $\rho(2 s) \leq c \rho(s), s>0$. It is easy to see that the radial weight $\rho(|x|) \in A_{1}$ if and only if

$$
\int_{0}^{r} s^{n-1} \rho(s)^{2 \tau} d s \leq C r^{n} \rho(r), \quad r>0 .
$$

Moreover, the $A_{1}$-bound of $\rho$ is bounded by a constant which depends only on $C$ in the preceding estimate and the doubling constant $c$ (see [St2]). 
It follows from (5.8) that $\widetilde{G}_{2}(s) \asymp|s|^{2-n}$ if $n \geq 3, \widetilde{G}_{2}(s) \asymp \log \frac{2}{|s|}$ if $n=2$, for $0<s<1$, and $\widetilde{G}_{2}(s) \asymp 1$ for $s \geq 1$. Hence, $\rho(|s|)=\widetilde{G}_{2}^{\tau}(s)$ is a radial nonincreasing kernel with the doubling property. Clearly, (5.14) holds if and only if $0<2 \tau<\frac{n}{n-2}$, and the $A_{1}$-bound of $\widetilde{G}_{2}^{\tau}$ depends only on $\tau$ and $n$.

By Jensen's inequality, $\widetilde{G}_{2}^{\tau_{1}} \in A_{1}$ implies $\widetilde{G}_{2}^{\tau_{2}} \in A_{1}$ if $\tau_{1} \geq \tau_{2}$. Hence, without loss of generality we may assume $1 \leq 2 \tau \leq \frac{n}{n-2}$. Then by Minkowski's integral inequality and the $A_{1}$-estimate for $\widetilde{G}_{2}^{2 \tau}$ established above, it follows: $M\left(\widetilde{P}^{2 \tau}\right)(x) \leq\left(\left(M \widetilde{G}_{2}^{2 \tau}\right)^{\frac{1}{2 \tau}} \star \mu(x)\right)^{2 \tau} \leq C(\tau, n)\left(\widetilde{G}_{2} \star \mu\right)^{2 \tau}(x)=C(\tau, n) \widetilde{P}^{2 \tau}(x)$.

Proposition 5.2. Let $n \geq 2$ and $1<2 \tau<\min \left(\frac{n}{n-2}, 2\right)$. Let $P=(1-\Delta)^{-1} \mu$, where $\mu \in M^{+}\left(\mathbb{R}^{n}\right)$ is a compactly supported Borel measure on $\mathbb{R}^{n}$. Then

$$
\left\|\nabla P^{\tau}\right\|_{L^{2}\left(\mathbb{R}^{n}\right)}^{2}=\frac{\tau^{2}}{2 \tau-1}\left(\int_{\mathbb{R}^{n}} P^{2 \tau-1} d \mu-\int_{\mathbb{R}^{n}} P^{2 \tau} d x\right),
$$

provided $P^{\tau} \in W^{1,2}\left(\mathbb{R}^{n}\right)$.

Remark 5.1. Suppose $\mu$ is the equilibrium measure of a compact set $e \subset \mathbb{R}^{n}$, so that $\mu(e)=\operatorname{cap}(e), P(x)=1 d \mu$-a.e., and $0 \leq P(x) \leq 1$ on $\mathbb{R}^{n}$. Then it is easy to see that $P^{\tau} \in W^{1,2}\left(\mathbb{R}^{n}\right)$ if $1<2 \tau<\min \left(\frac{n}{n-2}, 1\right)$, and (5.15) yields the estimates:

$$
\begin{aligned}
\left\|\nabla P^{\tau}\right\|_{L^{2}\left(\mathbb{R}^{n}\right)}^{2} & \leq \frac{\tau^{2}}{2 \tau-1} \operatorname{cap}(e), \\
\left\|P^{\tau}\right\|_{L^{2}\left(\mathbb{R}^{n}\right)}^{2} & \leq \operatorname{cap}(e) .
\end{aligned}
$$

Proof. The proof of Proposition 5.2 is based on a multiple integration by parts argument, and is analogous to the proof of Proposition 2.5 in [MV2]. Clearly,

$$
\Delta P=P-(1-\Delta) P=P-\mu
$$

in the sense of distributions. Furthermore, for $\tau>0$,

$$
\begin{aligned}
\Delta P^{\tau} & =\tau(\tau-1) P^{\tau-2}|\nabla P|^{2}+\tau \Delta P P^{\tau-1} \\
& =\tau(\tau-1) P^{\tau-2}|\nabla P|^{2}+\tau P^{\tau}-\tau P^{\tau-1} \mu .
\end{aligned}
$$

Using integration by parts and the preceding equation, we obtain:

$$
\begin{aligned}
\left\|\nabla P^{\tau}\right\|_{L^{2}\left(\mathbb{R}^{n}\right)}^{2} & =\int_{\mathbb{R}^{n}} \nabla P^{\tau} \cdot \nabla P^{\tau} d x \\
& =-\int_{\mathbb{R}^{n}} P^{\tau} \Delta P^{\tau} d x \\
& =-\tau(\tau-1) \int_{\mathbb{R}^{n}} P^{2 \tau-2}|\nabla P|^{2} d x-\tau \int_{\mathbb{R}^{n}} P^{2 \tau} d x+\tau \int_{\mathbb{R}^{n}} P^{2 \tau-1} d \mu .
\end{aligned}
$$


Here integration by parts is easily justified, as long as $1<2 \tau<\min \left(\frac{n}{n-2}, 1\right)$, by looking at the behavior of $P(x)$ and $\nabla P(x)$ at infinity:

$$
\begin{aligned}
P(x) & \asymp|x|^{\frac{1-n}{2}} e^{-|x|} \mu(e), & & |x| \rightarrow+\infty \\
|\nabla P(x)| & \leq c(n)|x|^{\frac{1-n}{2}} e^{-|x|} \mu(e), & & |x| \rightarrow+\infty .
\end{aligned}
$$

(See details in the proof of Lemma 4.3 in MV2].) Next, we use integration by parts again to obtain:

$$
\begin{aligned}
\int_{\mathbb{R}^{n}} P^{2 \tau-2}|\nabla P|^{2} d x & =\int_{\mathbb{R}^{n}} P^{2 \tau-2} \nabla P \cdot \nabla P d x \\
& =-\int_{\mathbb{R}^{n}} \Delta P P^{2 \tau-1} d x-(2 \tau-2) \int_{\mathbb{R}^{n}} P^{2 \tau-2}|\nabla P|^{2} d x .
\end{aligned}
$$

From the preceding equation we deduce:

$$
\begin{aligned}
(2 \tau-1) \int_{\mathbb{R}^{n}} P^{2 \tau-2}|\nabla P|^{2} d x & =-\int_{\mathbb{R}^{n}} \Delta P P^{2 \tau-1} d x \\
& =\int_{\mathbb{R}^{n}}(1-\Delta) P P^{2 \tau-1} d x-\int_{\mathbb{R}^{n}} P^{2 \tau} d x \\
& =\int_{\mathbb{R}^{n}} P^{2 \tau-1} d \mu-\int_{\mathbb{R}^{n}} P^{2 \tau} d x
\end{aligned}
$$

Combining the preceding equations, we finally have:

$$
\left\|\nabla P^{\tau}\right\|_{L^{2}\left(\mathbb{R}^{n}\right)}^{2}=\frac{\tau^{2}}{2 \tau-1}\left(\int_{\mathbb{R}^{n}} P^{2 \tau-1} d \mu-\int_{\mathbb{R}^{n}} P^{2 \tau} d x\right) .
$$

This completes the proof of Proposition 5.2.

We will need a more precise estimate of $\left\|P^{\tau}\right\|_{L^{2}\left(\mathbb{R}^{n}\right)}^{2}$ than (15.17) in Remark 5.1. Note that estimate (5.16) is sharp.

Proposition 5.3. Let $n \geq 2$, and $1 \leq 2 \tau<\frac{n}{n-2}$. Let $\mu \in M^{+}\left(\mathbb{R}^{n}\right)$ be a measure supported on a compact set $e \subset B_{\delta}\left(x_{0}\right)$, where $0<\delta \leq 1$. Let $P=G_{2} \star \mu$. Then the following estimates hold:

$$
\begin{array}{ll}
\int_{\mathbb{R}^{n}} P^{2 \tau} d x \leq c(n, \tau) \mu(e)^{2 \tau}, & n \geq 2 \\
\int_{B_{2 \delta}\left(x_{0}\right)} P^{2 \tau} d x \leq c(n, \tau) \delta^{n-(n-2) 2 \tau} \mu(e)^{2 \tau}, & n \geq 3 \\
\int_{B_{2 \delta}\left(x_{0}\right)} P^{2 \tau} d x \leq c(2, \tau) \delta^{2} \log ^{2 \tau}\left(\frac{2}{\delta}\right) \mu(e)^{2 \tau}, & n=2
\end{array}
$$

Remark 5.2. Estimates (5.18) $-(5.20)$ are sharp, and can be reversed. 
Proof. Suppose $e \subset B_{\delta}\left(x_{0}\right)$, where $0<\delta \leq 1$. Then

$$
\int_{\mathbb{R}^{n}} P^{2 \tau} d x=\int_{B_{2 \delta}\left(x_{0}\right)} P(x)^{2 \tau} d x+\int_{B_{2 \delta}\left(x_{0}\right)^{c}} P(x)^{2 \tau} d x=I+I I .
$$

Using Minkowski's integral inequality and the estimate $G_{2}(x) \leq c(n)|x|^{2-n}$ for $|x| \leq 3$ and $n \geq 3$, we get:

$$
\begin{aligned}
I & =c(n) \int_{B_{2 \delta}\left(x_{0}\right)}\left(\int_{e} G_{2}(x-t) d \mu(t)\right)^{2 \tau} d x \\
& \leq c(n, \tau) \int_{B_{2 \delta}\left(x_{0}\right)}\left(\int_{e} \frac{d \mu(t)}{|x-t|^{n-2}}\right)^{2 \tau} d x \\
& \leq c(n, \tau)\left\{\int_{e}\left(\int_{B_{2 \delta}\left(x_{0}\right)} \frac{d x}{|x-t|^{(n-2) 2 \tau}}\right)^{\frac{1}{2 \tau}} d \mu(t)\right\}^{2 \tau} .
\end{aligned}
$$

Since $2 \tau<\frac{n}{n-2}$, it follows:

$$
\int_{B_{2 \delta}\left(x_{0}\right)} \frac{d x}{|x-t|^{(n-2) 2 \tau}} \leq \int_{|x-t|<3 \delta} \frac{d x}{|x-t|^{(n-2) 2 \tau}} \leq c(n, \tau) \delta^{n-(n-2) 2 \tau} .
$$

Hence,

$$
I \leq c(n, \tau) \delta^{n-(n-2) 2 \tau} \mu(e)^{2 \tau}
$$

This proves (5.19). In the case $n=2$, similar estimates using Minkowski's integral inequality and $G_{2}(x) \asymp \log \frac{C}{|x|}$ as $|x| \rightarrow+0$, give (5.20).

To estimate $I I$, notice that

$$
\int_{\mathbb{R}^{n}} G_{2}(x)^{2 \tau} d x<+\infty, \quad 0<2 \tau<\frac{n}{n-2} .
$$

If $\left|x-x_{0}\right| \geq 2 \delta$ and $\left|x_{0}-t\right|<\delta$, it follows that $|x-t| \geq \frac{1}{2}\left|x-x_{0}\right|$, and $G_{2}(x-t) \leq G_{2}\left(\frac{1}{2}\left|x-x_{0}\right|\right)$. Hence,

$$
\begin{aligned}
I I & =\int_{B_{2 \delta}\left(x_{0}\right)}\left(\int_{e} G_{2}(x-t) d \mu(t)\right)^{2 \tau} d x \\
& \leq \mu(e)^{2 \tau} \int_{\mathbb{R}^{n}} G_{2}\left(\frac{1}{2}\left|x-x_{0}\right|\right)^{2 \tau} d x \leq C(\tau, n) \mu(e)^{2 \tau} .
\end{aligned}
$$

Combining the estimates for $I$ and $I I$, we complete the proof of (5.18).

The proof of the next proposition is contained in the proof of Theorem 4.2 in MV2. 
Proposition 5.4. ([MV2]). Let $1<2 \tau<\frac{n}{n-2}$. Then

$$
\left\|\nabla\left(w P^{-\tau}\right)\right\|_{L^{2}\left(\mathbb{R}^{n}\right)} \leq c(n, \tau)\left(\int_{\mathbb{R}^{n}}\left(|\nabla w|^{2}+|w|^{2}\right) P^{-2 \tau} d x\right)^{\frac{1}{2}} .
$$

Proposition 5.5. Let $e \subset B_{\delta}\left(x_{0}\right), 0<\delta \leq 1$, and $P(x)=(1-\Delta)^{-1} \mu$, where $\mu$ is the equilibrium measure associated with e. Let $1<2 \tau<\min \left(\frac{n}{n-2}, 2\right)$. Let $w=\nabla(1-\Delta)^{-1} \psi$, where $\psi \in C_{0}^{\infty}\left(B_{\delta}\left(x_{0}\right)\right), 0<\delta \leq 1$. Then the following estimates hold:

$$
\begin{array}{ll}
\int_{\mathbb{R}^{n}} \frac{|\nabla w|^{2}}{P^{2 \tau}} d x \leq C(n, \tau) \int_{B_{\delta}\left(x_{0}\right)} \frac{|\psi|^{2}}{P^{2 \tau}} d x & n \geq 2 ; \\
\int_{\mathbb{R}^{n}} \frac{|w|^{2}}{P^{2 \tau}} d x \leq C(n, \tau) \delta^{n-(n-2) 2 \tau} \int_{B_{\delta}\left(x_{0}\right)} \frac{|\psi|^{2}}{P^{2 \tau}} d x, & n \geq 3 ; \\
\int_{\mathbb{R}^{2}} \frac{|w|^{2}}{P^{2 \tau}} d x \leq C(2, \tau) \delta^{2} \log ^{2 \tau}\left(\frac{2}{\delta}\right) \int_{B_{\delta}\left(x_{0}\right)} \frac{|\psi|^{2}}{P^{2 \tau}} d x, & n=2 .
\end{array}
$$

Proof. Estimate (5.23) is actually contained in Lemma 4.3 MV2 where a detailed proof was given only in the case $n \geq 3$. For the sake of completeness, and since a similar argument is needed anyway in the proof of estimates (5.24) - (5.25), we give a proof of (5.23) here which is valid for $n \geq 2$.

We split the integral on the left-hand side of (5.23) into two parts:

$$
\int_{\mathbb{R}^{n}} \frac{|\nabla w|^{2}}{P^{2 \tau}} d x=\int_{B_{2}\left(x_{0}\right)} \frac{|\nabla w|^{2}}{P^{2 \tau}} d x+\int_{B_{2}\left(x_{0}\right)^{c}} \frac{|\nabla w|^{2}}{P^{2 \tau}} d x=I+I I,
$$

where $w=\nabla(1-\Delta)^{-1} \psi$, and $\psi \in C_{0}^{\infty}\left(B_{\delta}\left(x_{0}\right)\right), 0<\delta \leq 1$.

To estimate $I$, we begin by replacing $P^{2 \tau}$ above with $\widetilde{P}^{2 \tau}$, where $\widetilde{P}=\widetilde{G}_{2} \star \mu$ is the modified equilibrium potential, and

$$
P(x) \asymp \widetilde{P}(x) \quad \text { for } \quad x \in B_{2}\left(x_{0}\right) .
$$

Notice that $\nabla w=\nabla \nabla(1-\Delta)^{-1} \psi$, where $\nabla \nabla$ denotes the Hessian. Clearly,

$$
\nabla \nabla(1-\Delta)^{-1}=\left\{i R_{j} i R_{k} \Delta(1-\Delta)^{-1}\right\}, \quad j, k=1, \ldots, n,
$$

where $\left\{R_{j}\right\}_{j=1}^{n}$ are the Riesz transforms [St2], which are known to be bounded operators on $L^{2}\left(\mathbb{R}^{n}, \rho d x\right)$ with weights $\rho$ in the Muckenhoupt class $A_{2}$ defined by:

$$
\sup \left\{m_{B_{\delta}(x)}(\rho) m_{B_{\delta}(x)}\left(\rho^{-1}\right): \quad \delta>0, x \in \mathbb{R}^{n}\right\}<+\infty .
$$

Moreover, the operator norms of $R_{j}$ in $L^{2}\left(\mathbb{R}^{n}, \rho d x\right)$ are bounded by a constant which depends only on the $A_{2}$-bound of $\rho$ given by the preceding formula.

By Proposition [5.1, the weight $\rho=\widetilde{P}^{2 \tau}$ is in the Muckenhoupt class $A_{1}$, and hence to $A_{2}$ (see $\left[\mathrm{St2}\right.$ ), and its $A_{2}$-bound depends only on $\tau, n$. Hence, 
$\rho^{-1}=\widetilde{P}^{-2 \tau} \in A_{2}$, and
$\quad\left\|R_{j} f\right\|_{L^{2}\left(\mathbb{R}^{n}, \widetilde{P}^{-2 \tau} d x\right)} \leq C(\tau, n)\|f\|_{L^{2}\left(\mathbb{R}^{n}, \widetilde{P}^{-2 \tau} d x\right)}, \quad \forall f \in L^{2}\left(\mathbb{R}^{n}, \widetilde{P}^{-2 \tau} d x\right)$, for every $j=1, \ldots, n$. It follows:

$$
I \leq c(\tau, n) \int_{B_{2}\left(x_{0}\right)} \frac{|\nabla w|^{2}}{\widetilde{P}^{2 \tau}} d x \leq C(\tau, n) \int_{\mathbb{R}^{n}} \frac{\left|\Delta(1-\Delta)^{-1} \psi\right|^{2}}{\widetilde{P}^{2 \tau}} d x .
$$

It remains to notice that $\Delta(1-\Delta)^{-1}=1-(1-\Delta)^{-1}$ is a Fourier multiplier operator on the space $L^{2}\left(\mathbb{R}^{n}, \rho d x\right)$ for any weight $\rho \in A_{2}$, and its norm depends only on the Muckenhoupt bound of $\rho$. (See, e.g., MV2] or [MV4.) Thus, for $\rho=\widetilde{P}^{-2 \tau}$, we have:

$$
I \leq C(n, \tau) \int_{\mathbb{R}^{n}} \frac{|\psi|^{2}}{\widetilde{P}^{2 \tau}} d x \leq C(\tau, n) \int_{B_{\delta}\left(x_{0}\right)} \frac{|\psi|^{2}}{P^{2 \tau}} d x,
$$

since $\widetilde{P}^{2 \tau}(x) \asymp P^{2 \tau}(x)$ on $\operatorname{supp} \psi \subset B_{\delta}\left(x_{0}\right)$, where $0<\delta \leq 1$.

The term II can be estimated directly using known inequalities for derivatives of Bessel potentials (see, e.g., $[\mathrm{AH}]$, Sec. 1.2.5):

$$
\left|\nabla^{k} G_{2}(x)\right| \leq C(n)|x|^{\frac{1-n}{2}} e^{-|x|}, \quad|x| \geq 1,
$$

where $k=0,1,2$. Notice that both $\psi$ and $\mu$ are supported in $B_{\delta}\left(x_{0}\right) \subset B_{1}\left(x_{0}\right)$. Hence, by the preceding estimate,

$\left|\nabla \nabla(1-\Delta)^{-1} \psi(x)\right| \leq C(n)\left|x-x_{0}\right|^{\frac{1-n}{2}} e^{-\left|x-x_{0}\right|}\|\psi\|_{L^{1}\left(B_{\delta}\left(x_{0}\right)\right.}, \quad \forall x \in B_{2}\left(x_{0}\right)^{c}$.

From the lower estimate $G_{2}(x) \geq c(n)|x|^{\frac{1-n}{2}} e^{-|x|}$ for $|x| \geq 1$, we have:

$$
P(x)=\int_{e} G_{2}(x-y) d \mu(y) \geq c(n)\left|x-x_{0}\right|^{\frac{1-n}{2}} e^{-\left|x-x_{0}\right|} \mu(e), \quad \forall x \in B_{2}\left(x_{0}\right)^{c} .
$$

Since $\mu(e)=\operatorname{cap}(e)>0$, it follows:

$$
\begin{aligned}
& I I=\int_{B_{2}\left(x_{0}\right)^{c}} \frac{\left|\nabla \nabla(1-\Delta)^{-1} \psi(x)\right|^{2}}{P^{2 \tau}(x)} d x \leq C(\tau, n)\|\psi\|_{L^{1}\left(B_{\delta}\left(x_{0}\right)\right.}^{2} \operatorname{cap}(e)^{-2 \tau} \\
& \times \int_{B_{2}\left(x_{0}\right)^{c}}\left|x-x_{0}\right|^{(1-n)(1-\tau)} e^{-(2-2 \tau)\left|x-x_{0}\right|} d x \leq C(\tau, n)\|\psi\|_{L^{1}\left(B_{\delta}\left(x_{0}\right)\right)}^{2} \operatorname{cap}(e)^{-2 \tau},
\end{aligned}
$$

where the constant $C(\tau, n)$ is finite as long as $\tau<1$.

Now by Schwarz's inequality,

$$
\|\psi\|_{L^{1}\left(B_{\delta}\left(x_{0}\right)\right)}^{2} \leq \int_{B_{\delta}\left(x_{0}\right)} \frac{|\psi(x)|^{2}}{P^{2 \tau}(x)} d x \int_{B_{\delta}\left(x_{0}\right)} P^{2 \tau}(x) d x .
$$

By Proposition 5.3. $\int_{\mathbb{R}^{n}} P^{2 \tau} d x \leq c(\tau, n) \operatorname{cap}(e)^{2 \tau}$. Combining these estimates, we obtain:

$$
I I \leq C(\tau, n) \int_{B_{\delta}\left(x_{0}\right)} \frac{|\psi(x)|^{2}}{P^{2 \tau}(x)} d x .
$$


This completes the proof of estimate (5.23).

We now prove estimates $(\underline{5.24})-(15.25)$. Splitting the integral $\int_{\mathbb{R}^{n}} \frac{|w|^{2}}{P^{2 \tau}} d x$ into two parts depending on $\delta$, we have:

$$
\int_{\mathbb{R}^{n}} \frac{|w|^{2}}{P^{2 \tau}} d x=\int_{B_{2 \delta}\left(x_{0}\right)} \frac{|w|^{2}}{P^{2 \tau}} d x+\int_{B_{2 \delta}\left(x_{0}\right)^{c}} \frac{|w|^{2}}{P^{2 \tau}} d x=I+I I,
$$

where as before $w=\nabla(1-\Delta)^{-1} \psi$, and $\psi \in C_{0}^{\infty}\left(B_{\delta}\left(x_{0}\right)\right), 0<\delta \leq 1$. Using the estimate $([\mathrm{AH}]$, Sec. 1.2.5):

$$
\left|\nabla G_{2}(x)\right| \leq C(n)|x|^{1-n}, \quad|x| \leq 3, \quad n \geq 2,
$$

we have:

$$
|w(x)|=\left|\nabla(1-\Delta)^{-1} \psi(x)\right| \leq \int_{B_{\delta}\left(x_{0}\right)} \frac{|\psi(t)|}{|x-t|^{n-1}} d t, \quad \forall x \in B_{2 \delta}\left(x_{0}\right) .
$$

We next use a version of Hedberg's inequality (see [AH]):

$$
\int_{B_{\delta}\left(x_{0}\right)} \frac{|\psi(t)|}{|x-t|^{n-1}} d t \leq \sum_{j=0}^{+\infty} \delta 2^{-j} \int_{\delta 2^{-j-1}<|x-t| \leq \delta 2^{-j}} \frac{|\psi(t)|}{|x-t|^{n}} d t \leq C(n) \delta M \psi(x),
$$

for every $x \in B_{2 \delta}\left(x_{0}\right)$. Here $M$ is the Hardy-Littlewood maximal function.

Since $P^{2 \tau} \asymp \widetilde{P}^{2 \tau}$ on $B_{2 \delta}\left(x_{0}\right)$ we have:

$$
\int_{B_{2 \delta}\left(x_{0}\right)} \frac{|w|^{2}}{P^{2 \tau}} d x \leq C(\tau, n) \delta^{2} \int_{\mathbb{R}^{n}} \frac{|M \psi|^{2}}{\widetilde{P}^{2 \tau}} d x \leq C(\tau, n) \delta^{2} \int_{B_{\delta}\left(x_{0}\right)} \frac{|\psi|^{2}}{P^{2 \tau}} d x .
$$

Here we have used the fact that $M$ is a bounded operator on $L^{2}\left(\mathbb{R}^{n}, \rho\right)$ with the weight $\rho=\widetilde{P}^{-2 \tau} \in A_{2}$, and its operator norm is bounded by a constant which depends only on the $A_{2}$-bound of $P^{-2 \tau}$ (see [St2]). Hence, by Proposition 5.1] it is bounded by $C(\tau, n)$. Note that we can use $P^{2 \tau}$ in place of $\widetilde{P}^{2 \tau}$ on $\operatorname{supp} \psi \subset$ $B_{\delta}\left(x_{0}\right)$ since $0<\delta \leq 1$.

Thus,

$$
I \leq C(n, \tau) \delta^{2} \int_{B_{\delta}\left(x_{0}\right)} \frac{|\psi|^{2}}{P^{2 \tau}} d x, \quad n \geq 2 .
$$

To estimate $I I$, notice that

$$
\left|\nabla(1-\Delta)^{-1} \psi(x)\right| \leq \int_{B_{\delta}\left(x_{0}\right)}\left|\nabla G_{2}(x-t)\right||\psi(t)| d t, \quad \forall x \in B_{2 \delta}\left(x_{0}\right)^{c},
$$

where $|x-t| \geq\left|x-x_{0}\right|-\delta \geq \frac{1}{2}\left|x-x_{0}\right|$. Using estimates for derivatives of Bessel kernels ([AH], Sec. 1.2.5), we have:

$$
\begin{aligned}
\left|\nabla G_{2}(x-t)\right| \leq C(n) G_{1}\left(x-x_{0}\right), & & \left|x-x_{0}\right| \leq 1 \\
\left|\nabla G_{2}(x-t)\right| \leq C(n) G_{2}\left(x-x_{0}\right), & & \left|x-x_{0}\right| \geq 1,
\end{aligned}
$$

where $x \in B_{2 \delta}\left(x_{0}\right)^{c}$ and $t \in B_{\delta}\left(x_{0}\right), 0<\delta \leq 1$. 
Since $\mu(e)=\operatorname{cap}(e)>0$, and $|x-t| \leq\left|x-x_{0}\right|+\delta$, we estimate:

$$
P(x)=\int_{e} G_{2}(x-t) d \mu(t) \geq c(n) G_{2}\left(x-x_{0}\right) \operatorname{cap}(e), \quad \forall x \in B_{2 \delta}\left(x_{0}\right)^{c} .
$$

It follows:

$$
\begin{aligned}
I I & =\int_{B_{2 \delta}\left(x_{0}\right)^{c}} \frac{\left|\nabla(1-\Delta)^{-1} \psi(x)\right|^{2}}{P(x)^{2 \tau}} d x \leq C(\tau, n)\|\psi\|_{L^{1}\left(B_{\delta}\left(x_{0}\right)\right)}^{2} \operatorname{cap}(e)^{-2 \tau} \\
& \times\left(\int_{B_{1}\left(x_{0}\right)} \frac{G_{1}\left(x-x_{0}\right)^{2}}{G_{2}\left(x-x_{0}\right)^{2 \tau}} d x+\int_{B_{1}\left(x_{0}\right)^{c}} G_{2}\left(x-x_{0}\right)^{2-2 \tau} d x\right) .
\end{aligned}
$$

It is easy to see that, for $2 \tau \in(1,2)$,

$$
\int_{B_{1}\left(x_{0}\right)} \frac{G_{1}\left(x-x_{0}\right)^{2}}{G_{2}\left(x-x_{0}\right)^{2 \tau}} d x+\int_{B_{1}\left(x_{0}\right)^{c}} G_{2}\left(x-x_{0}\right)^{2-2 \tau} d x<+\infty .
$$

Indeed, estimates for Bessel kernels yield:

$$
\begin{aligned}
\frac{G_{1}(x)^{2}}{G_{2}(x)^{2 \tau}} & \leq C(\tau, n)|x|^{2 \tau(n-2)-2(n-1)}, \quad|x| \leq 1, \quad n \geq 3 ; \\
\frac{G_{1}(x)^{2}}{G_{2}(x)^{2 \tau}} & \leq C(\tau, n)|x|^{-2} \log ^{-2 \tau} \frac{2}{|x|}, \quad|x| \leq 1, \quad n=2 ; \\
G_{2}(x)^{2-2 \tau} & \leq C(\tau, n)|x|^{(1-n)(1-\tau)} e^{(2 \tau-2)|x|}, \quad|x| \geq 1, \quad n \geq 2,
\end{aligned}
$$

where the exponents satisfy the inequalities $2 \tau(n-2)-2(n-1)>-n$ in the case $n \geq 3$ and $-2 \tau<-1$ in the case $n=2$ for $|x| \leq 1$; and $2 \tau-2<0$ for $|x| \geq 1$. Thus,

$$
I I \leq C(\tau, n)\|\psi\|_{L^{1}\left(B_{\delta}\left(x_{0}\right)\right)}^{2} \operatorname{cap}(e)^{-2 \tau} .
$$

Now by Schwarz's inequality,

$$
\|\psi\|_{L^{1}\left(B_{\delta}\left(x_{0}\right)\right)}^{2} \leq \int_{B_{\delta}\left(x_{0}\right)} \frac{|\psi|^{2}}{P^{2 \tau}} d x \int_{B_{\delta}\left(x_{0}\right)} P^{2 \tau} d x,
$$

and by Proposition 5.3 ,

$$
\begin{array}{ll}
\int_{B_{\delta}\left(x_{0}\right)} P^{2 \tau} d x \leq C(\tau, n) \delta^{n-(n-2) 2 \tau} \operatorname{cap}(e)^{2 \tau}, & n \geq 3 \\
\int_{B_{\delta}\left(x_{0}\right)} P^{2 \tau} d x \leq C(\tau, 2) \delta^{2} \log ^{2 \tau}\left(\frac{2}{\delta}\right) \operatorname{cap}(e)^{2 \tau}, & n=2 .
\end{array}
$$

Combining these estimates, we obtain:

$$
\begin{array}{ll}
I I \leq C(\tau, n) \delta^{n-(n-2) 2 \tau} \int_{B_{\delta}\left(x_{0}\right)} \frac{|\psi|^{2}}{P^{2 \tau}} d x, & n \geq 3 ; \\
I I \leq C(\tau, 2) \delta^{2} \log ^{2 \tau}\left(\frac{2}{\delta}\right) \int_{B_{\delta}\left(x_{0}\right)} \frac{|\psi|^{2}}{P^{2 \tau}} d x, & n=2 .
\end{array}
$$


Since $2 \tau>1$, obviously $\delta^{2} \leq \delta^{n-(n-2) 2 \tau}$ if $n \geq 3$, and $\delta^{2} \leq \delta^{2} \log ^{2 \tau}\left(\frac{2}{\delta}\right)$ in the case $n=2$. Thus, the above inequalities for $I$ and $I I$ yield the desired estimate for $\int_{\mathbb{R}^{n}} \frac{|w|^{2}}{P^{2 \tau}} d x$.

The proof of the next proposition is very similar to that of Proposition 5.5. with obvious changes in the orders of Bessel potentials, and so we omit it.

Proposition 5.6. Let $1<2 \tau<\min \left(\frac{n}{n-2}, 2\right)$. Let $u=(1-\Delta)^{-1} \psi$, where $\psi \in C_{0}^{\infty}\left(B_{\delta}\left(x_{0}\right)\right), 0<\delta \leq 1$. Then

$$
\begin{aligned}
& \int_{\mathbb{R}^{n}} \frac{|u|^{2}}{P^{2 \tau}} d x \leq C(\tau, n) \delta^{n-(n-2) 2 \tau} \int_{B_{\delta}\left(x_{0}\right)} \frac{|\psi|^{2}}{P^{2 \tau}} d x, \quad n \geq 3 ; \\
& \int_{\mathbb{R}^{2}} \frac{|u|^{2}}{P^{2 \tau}} d x \leq C(\tau, 2) \delta^{2} \log ^{2 \tau}\left(\frac{2}{\delta}\right) \int_{B_{\delta}\left(x_{0}\right)} \frac{|\psi|^{2}}{P^{2 \tau}} d x, \quad n=2 ;
\end{aligned}
$$

We can now complete the proof of the implication (5.4) $\Rightarrow(5.5)$. Let $e \subset$ $B_{\delta}\left(x_{0}\right)$ be a set of positive capacity, and let $\vec{\psi}=\left\{\psi_{k}\right\}_{k=1}^{n}$, where $\psi_{k} \in$ $C_{0}^{\infty}\left(B_{\delta}\left(x_{0}\right)\right), k=1, \ldots, n$, and $0<\delta \leq 1$. Denote by $P=G_{2} \star \mu$ the potential of the equilibrium measure $\mu$ associated with $e$ which satisfies properties (a)-(e) listed above. Pick $\tau$ so that (15.12) holds.

We next apply (5.4) with $u=P^{\tau}$ and $v=\frac{w}{P^{\tau}}$, where $w=\operatorname{div}(1-\Delta)^{-1} \vec{\psi}$. (Since $u, v \in W^{1,2}\left(\mathbb{R}^{n}\right)$, we can actually use that inequality for mollifiers of $u$ and $v$ and then pass to the limit as in MV2.) We obtain:

$$
\begin{aligned}
|\langle Q, w\rangle| & \leq \epsilon\left(\left\|\nabla P^{\tau}\right\|_{L^{2}\left(\mathbb{R}^{n}\right)}^{2}+\frac{C(\epsilon)}{\epsilon}\left\|P^{\tau}\right\|_{L^{2}\left(\mathbb{R}^{n}\right)}^{2}\right)^{\frac{1}{2}} \\
& \times\left(\left\|\nabla\left(\frac{w}{P^{\tau}}\right)\right\|_{L^{2}\left(\mathbb{R}^{n}\right)}^{2}+\frac{C(\epsilon)}{\epsilon}\left\|\frac{w}{P^{\tau}}\right\|_{L^{2}\left(\mathbb{R}^{n}\right)}^{2}\right)^{\frac{1}{2}} .
\end{aligned}
$$

Let us assume that $n \geq 3$. From the preceding inequality where $w=$ $\operatorname{div}(1-\Delta)^{-1} \vec{\psi}$, we deduce using Propositions 5.2 5.6 .

$$
\begin{aligned}
\left|\left\langle Q, \operatorname{div}(1-\Delta)^{-1} \vec{\psi}\right\rangle\right| & \leq c(n, \tau) \epsilon\left(\operatorname{cap}(e)+\operatorname{cap}(e)^{2 \tau} \frac{C(\epsilon)}{\epsilon}\right)^{\frac{1}{2}} \\
& \times\left(1+\delta^{n-(n-2) 2 \tau} \frac{C(\epsilon)}{\epsilon}\right)^{\frac{1}{2}}\left(\int \frac{|\vec{\psi}|^{2}}{P^{2 \tau}} d x\right)^{\frac{1}{2}}
\end{aligned}
$$

for all $\vec{\psi} \in C_{0}^{\infty}\left(B_{\delta}\left(x_{0}\right)\right)^{n}$.

Letting $\vec{\Gamma}=-\nabla(1-\Delta)^{-1} Q$, we have:

$$
\left\langle Q, \operatorname{div}(1-\Delta)^{-1} \vec{\psi}\right\rangle=\langle\vec{\Gamma}, \vec{\psi}\rangle,
$$


where $\vec{\Gamma} \in L_{\text {loc }}^{2}\left(\mathbb{R}^{n}\right)^{n}$. (This formal equation was justified in the sense of distributions in MV2 under a weaker assumption that $Q$ is form bounded with respect to $-\Delta$.)

Now taking the supremum over all $\vec{\psi} \in C_{0}^{\infty}\left(B_{\delta}\left(x_{0}\right)\right)^{n}$, we deduce, as in MV2:

$$
\begin{aligned}
\int_{B_{\delta}\left(x_{0}\right)}|\vec{\Gamma}|^{2} P^{2 \tau} d x & \leq c(n, \tau) \epsilon^{2}\left(\operatorname{cap}(e)+\operatorname{cap}(e)^{2 \tau} \frac{C(\epsilon)}{\epsilon}\right) \\
& \times\left(1+\delta^{n-(n-2) 2 \tau} \frac{C(\epsilon)}{\epsilon}\right) .
\end{aligned}
$$

Since $P(x) \geq 1$ quasieverywhere, and hence $d x$-a.e. on $e[\mathrm{AH}$, it follows that, for every compact set $e \subset B_{\delta}\left(x_{0}\right)$ of positive capacity,

$$
\int_{e}|\vec{\Gamma}|^{2} d x \leq c(n, \tau) \epsilon^{2}\left(\operatorname{cap}(e)+\operatorname{cap}(e)^{2 \tau} \frac{C(\epsilon)}{\epsilon}\right)\left(1+\delta^{n-(n-2) 2 \tau} \frac{C(\epsilon)}{\epsilon}\right) .
$$

Thus,

$$
\frac{\int_{e}|\vec{\Gamma}|^{2} d x}{\operatorname{cap}(e)} \leq c(n, \tau) \epsilon^{2}\left(1+\operatorname{cap}(e)^{2 \tau-1} \frac{C(\epsilon)}{\epsilon}\right)\left(1+\delta^{n-(n-2) 2 \tau} \frac{C(\epsilon)}{\epsilon}\right) .
$$

Since cap $(e)^{2 \tau-1} \leq c(n, \tau) \delta^{(n-2)(2 \tau-1)}$, it follows that

$$
\frac{\int_{e}|\vec{\Gamma}|^{2} d x}{\operatorname{cap}(e)} \leq c(n, \tau) \epsilon^{2}\left(1+\delta^{(n-2)(2 \tau-1)} \frac{C(\epsilon)}{\epsilon}\right)\left(1+\delta^{n-(n-2) 2 \tau} \frac{C(\epsilon)}{\epsilon}\right) .
$$

Taking the supremum over all $e$ and letting $\delta \rightarrow+0$, we obtain:

$$
\lim _{\delta \rightarrow+0} \sup _{e: \operatorname{diam} e \leq \delta} \frac{\int_{e}|\vec{\Gamma}|^{2} d x}{\operatorname{cap}(e)} \leq \epsilon
$$

The case $n=2$ requires usual modifications where a logarithmic term appears, as in Proposition 5.3.

A similar argument using Proposition [5.6 shows that the same inequality holds with $|\gamma|$ in place of $|\vec{\Gamma}|^{2}$. This proves (15.5), which yields (15.2). The proof of Theorem I is complete.

Theorem II stated in Sec. 2 is an immediate consequence of Theorem I combined with Theorem 4.1 and Corollary 4.3.

\section{FORM SUBORDINATION CRITERIA}

Suppose $Q \in \mathcal{D}^{\prime}\left(\mathbb{R}^{n}\right), n \geq 2$, and $\beta>0$. In this section we study quadratic form inequalities of Trudinger type; i.e., we characterize $Q$ for which there exist positive constants $c$ and $\epsilon_{0}$ such that, for every $\epsilon \in\left(0, \epsilon_{0}\right)$,

$$
|\langle Q u, u\rangle| \leq \epsilon|| \nabla u\left\|_{L^{2}\left(\mathbb{R}^{n}\right)}^{2}+c \epsilon^{-\beta}\right\| u \|_{L^{2}\left(\mathbb{R}^{n}\right)}^{2}, \quad \forall u \in C_{0}^{\infty}\left(\mathbb{R}^{n}\right) .
$$


By Corollary 3.3, this is equivalent to the existence of $C>0$ and $\delta_{0}>0$ such that

$$
|\langle Q u, u\rangle| \leq C \delta^{\frac{2}{1+\beta}}\|\nabla u\|_{L^{2}}^{2}, \quad \forall u \in C_{0}^{\infty}\left(B_{\delta}\left(x_{0}\right)\right),
$$

for all $\delta \in\left(0, \delta_{0}\right)$ and $x_{0} \in \mathbb{R}^{n}$. Moreover, if $\epsilon_{0}=1$ then one may set $\delta_{0}=1$; if $\epsilon_{0}=+\infty$ then $\delta_{0}=+\infty$, and the converse is also true.

We are now in a position to prove the following theorem which combines Theorems IV and V stated in Sec. 2.

Theorem 6.1. Let $Q \in \mathcal{D}^{\prime}\left(\mathbb{R}^{n}\right), n \geq 2$, and $\beta>1$. Then the following statements hold.

(i) Suppose there exist $\delta_{0}>0$, and $\vec{\Gamma} \in L_{\text {loc }}^{2}\left(\mathbb{R}^{n}\right)^{n}, \gamma \in L_{\text {loc }}^{2}$ such that

$$
Q=\operatorname{div} \vec{\Gamma}+\gamma
$$

where $\vec{\Gamma}$ and $\gamma$ satisfy respectively the inequalities:

$$
\begin{array}{ll}
\int_{B_{\delta}\left(x_{0}\right)}|\vec{\Gamma}(x)|^{2} d x \leq C_{1} \delta^{n-2+\frac{4}{1+\beta}}, & 0<\delta<\delta_{0}, \\
\int_{B_{\delta}\left(x_{0}\right)}|\gamma(x)| d x \leq C_{2} \delta^{n-2+\frac{2}{1+\beta}}, & 0<\delta<\delta_{0},
\end{array}
$$

with the constants $C_{1}, C_{2}$ which do not depend on $x_{0}$ and $\delta$. Then there exist $c>0$ and $\epsilon_{0}>0$ such that (6.1) holds for every $\epsilon \in\left(0, \epsilon_{0}\right)$.

(ii) Conversely, suppose (6.1) holds for every $\epsilon \in\left(0, \epsilon_{0}\right)$. Then $Q$ can be represented in the form (6.3) where $\vec{\Gamma} \in \mathbf{L}_{\text {loc }}^{2}\left(\mathbb{R}^{n}\right), \gamma \in L_{\text {loc }}^{2}\left(\mathbb{R}^{n}\right)$ satisfy respectively conditions (6.4) and (6.5).

(iii) If $\epsilon_{0}=1$, then one can set

$$
\vec{\Gamma}=-\nabla(1-\Delta)^{-1} Q, \quad \gamma=(1-\Delta)^{-1} Q
$$

in (6.3), where $\delta_{0}=1$ in (6.4), (6.5).

If $\epsilon_{0}=+\infty$, then one can set

$$
\vec{\Gamma}=\nabla \Delta^{-1} Q, \quad \gamma=0,
$$

in (6.3), where $\delta_{0}=+\infty$ in (6.4), (6.5).

Proof. We first prove statement (i). Suppose $\beta>1$ and $u \in C_{0}^{\infty}\left(B_{\delta}\left(x_{0}\right)\right)$. Suppose that $Q$ is represented in the form (6.3), and estimates (6.4), (6.5) hold for every $\delta>0$, i.e., $\delta_{0}=+\infty$. Applying the multiplicative inequality for nonnegative measures ([M2], Theorem 1.4.7) to $|\vec{\Gamma}|^{2} d x$ and $|\gamma| d x$ respectively, we get:

$$
\int_{\mathbb{R}^{n}}|\vec{\Gamma}(x)|^{2}|u(x)|^{2} d x \leq C_{1}\|\nabla u\|_{L^{2}}^{\frac{2(\beta-1)}{1+\beta}}\|u\|_{L^{2}}^{\frac{4}{1+\beta}}
$$


and

$$
\int_{\mathbb{R}^{n}}|\gamma(x)||u(x)|^{2} d x \leq C_{2}\|\nabla u\|_{L^{2}}^{\frac{2 \beta}{1+\beta}}\|u\|_{L^{2}}^{\frac{2}{1+\beta}}
$$

Hence,

$$
\begin{aligned}
|\langle Q u, u\rangle| & \leq|\langle\vec{\Gamma} u, \nabla u\rangle|+|\langle\gamma u, u\rangle| \\
& \leq\|\vec{\Gamma} u\|_{L^{2}}\|\nabla u\|_{L^{2}}+\left\|\gamma|u|^{2}\right\|_{L^{1}} \\
& \leq C_{1}^{\frac{1}{2}}\|\nabla u\|_{L^{2}}^{1+\frac{\beta-1}{1+\beta}}\|u\|_{L^{2}}^{\frac{2}{1+\beta}}+C_{2}\|\nabla u\|_{L^{2}}^{\frac{2 \beta}{1+\beta}}\|u\|_{L^{2}}^{\frac{2}{1+\beta}} .
\end{aligned}
$$

Combining the preceding estimates with the inequality (3.3),

$$
\|u\|_{L^{2}} \leq c(n) \delta\|\nabla u\|_{L^{2}}, \quad u \in C_{0}^{\infty}\left(B_{\delta}\left(x_{0}\right)\right),
$$

we obtain:

$$
|\langle Q u, u\rangle| \leq C \delta^{\frac{2}{1+\beta}}|| \nabla u \|_{L^{2}}, \quad u \in C_{0}^{\infty}\left(B_{\delta}\left(x_{0}\right)\right) .
$$

By Lemma 3.1 this implies (6.1).

If $\delta_{0}=1$, then in the proof above one has to replace $\|\nabla u\|_{L^{2}}$ with the inhomogeneous Sobolev norm $\|u\|_{W^{1,2}}$, and apply Corollary 1, Sec. 1.4.7 [M2] together with (3.3). For an arbitrary $\delta_{0}$, one can use $\|\nabla u\|_{L^{2}}+\delta_{0}^{-1}\|u\|_{L^{2}}$ in place of $\|u\|_{W^{1,2}}$ and the corresponding analogue of the multiplicative inequality for positive measures in $\mathrm{M} 2$. This completes the proof of statement (i).

We now prove statement (ii). We first establish a localized version of (ii) which holds for every $\beta>0$. Let $\eta_{\delta, x_{0}}(x)=\eta\left(\frac{x-x_{0}}{\delta}\right)$ where $\eta \in C_{0}^{\infty}\left(B_{1}(0)\right)$ is a smooth cut-off function such that $|\eta(x)| \leq 1$ and $|\nabla \eta(x)| \leq 1$ for $x \in B_{1}(0)$. It is worthwhile to observe that in all localized estimates below the constants will not depend on a particular choice of $\eta$.

Proposition 6.2. Suppose $Q \in \mathcal{D}^{\prime}\left(\mathbb{R}^{n}\right), n \geq 2$, and $\beta>0$. Suppose that (6.2) holds for every $\delta \in\left(0, \delta_{0}\right)$, where either $\delta_{0}=1$ or $\delta_{0}=+\infty$. Then the following inequalities hold:

$$
|\langle Q u, v\rangle| \leq c \delta^{\frac{2}{1+\beta}}\|\nabla u\|_{L^{2}}\|\nabla v\|_{L^{2}}, \quad u, v \in C_{0}^{\infty}\left(B_{\delta}\left(x_{0}\right)\right),
$$

and, in particular,

$$
\left|\left\langle Q \eta_{\delta, x_{0}}, v\right\rangle\right| \leq c \delta^{\frac{2}{1+\beta}+\frac{n-2}{2}}\|\nabla v\|_{L^{2}}, \quad v \in C_{0}^{\infty}\left(B_{\delta}\left(x_{0}\right)\right) .
$$

Moreover, if $\delta>\delta_{0}=1$, then

$$
\left|\left\langle Q \eta_{\delta, x_{0}}, v\right\rangle\right| \leq c \delta^{2+\frac{n-2}{2}}\|\nabla v\|_{L^{2}}, \quad v \in C_{0}^{\infty}\left(B_{\delta}\left(x_{0}\right)\right) .
$$

Proof. Clearly, (6.8) follows from (6.2) by the polarization identity. Letting $u=\eta_{\delta, x_{0}}$ in (6.8) one deduces (6.9). Finally, in the case $\delta_{0}=1$ and $\delta>1$, 
(6.10) follows by using a polarized form of (6.1) where one sets $\epsilon=1$, and $u=\eta_{\delta, x_{0}}, v \in C_{0}^{\infty}\left(B_{\delta}\left(x_{0}\right)\right)$, together with estimate (3.3):

$$
\begin{aligned}
\left|\left\langle Q \eta_{\delta, x_{0}}, v\right\rangle\right| & \leq \sqrt{\left\|\nabla \eta_{\delta, x_{0}}\right\|_{L^{2}}^{2}+c\left\|\eta_{\delta, x_{0}}\right\|_{L^{2}}^{2}} \sqrt{\|\nabla v\|_{L^{2}}^{2}+c\|v\|_{L^{2}}^{2}} \\
& \leq C \sqrt{\delta^{n-2}+c \delta^{n}} \sqrt{\|\nabla v\|_{L^{2}}^{2}+c \delta^{2}\|\nabla v\|_{L^{2}}^{2}} \\
& \leq C \delta^{2+\frac{n-2}{2}}\|\nabla v\|_{L^{2}}
\end{aligned}
$$

We now establish certain localized versions of the necessary condition for (6.1) and (6.2).

Proposition 6.3. Suppose $Q \in \mathcal{D}^{\prime}\left(\mathbb{R}^{n}\right), n \geq 2$, and $\beta>0$. Suppose that (6.2) holds for every $\delta \in\left(0, \delta_{0}\right)$, where either $\delta_{0}=1$ or $\delta_{0}=+\infty$. Let $\vec{\Gamma}$ and $\gamma$ be defined by (6.6) if $\delta_{0}=1$, or by (6.7) if $\delta_{0}=+\infty$.

(i) For $n \geq 3$,

$$
\int_{\mathbb{R}^{n}}\left|\nabla(1-\Delta)^{-1}\left(\eta_{\delta, x_{0}} Q\right)\right|^{2} d x \leq c \delta^{\frac{4}{1+\beta}+n-2}, \quad 0<\delta \leq 1,
$$

and

$$
\int_{\mathbb{R}^{n}}\left|(1-\Delta)^{-1}\left(\eta_{\delta, x_{0}} Q\right)\right| d x \leq c \delta^{\frac{2}{1+\beta}+n-2}, \quad 0<\delta \leq 1 .
$$

(ii) For $n \geq 3$ and $\delta_{0}=+\infty$,

$$
\int_{\mathbb{R}^{n}}\left|\nabla \Delta^{-1}\left(\eta_{\delta, x_{0}} Q\right)\right|^{2} d x \leq c \delta^{\frac{4}{1+\beta}+n-2}, \quad 0<\delta<+\infty .
$$

(iii) For $n \geq 2$,

$$
\int_{B_{\delta}\left(x_{0}\right)}\left|\nabla(1-\Delta)^{-1}\left(\eta_{\delta, x_{0}} Q\right)\right|^{2} d x \leq c \delta^{\frac{4}{1+\beta}+n-2}, \quad 0<\delta \leq 1,
$$

and

$$
\int_{\mathbb{R}^{n}}\left|(1-\Delta)^{-1}\left(\eta_{\delta, x_{0}} Q\right)\right| d x \leq c \delta^{\frac{2}{1+\beta}+n-2}, \quad 0<\delta \leq 1 .
$$

(iv) For $n \geq 2$ and $\delta_{0}=+\infty$,

$$
\int_{B_{\delta}\left(x_{0}\right)}\left|\nabla \Delta^{-1}\left(\eta_{\delta, x_{0}} Q\right)\right|^{2} d x \leq c \delta^{\frac{4}{1+\beta}+n-2}, \quad 0<\delta<+\infty .
$$

Proof. We first consider the case $\delta_{0}=1$. The homogeneous case $\delta_{0}=+\infty$ will be treated in a similar way.

We pick another cut-off function $\zeta \in C_{0}^{\infty}\left(B_{1}(0)\right)$ such that $\zeta(x) \eta(x)=\eta(x)$, and let $\zeta_{\delta, x_{0}}(x)=\zeta\left(\frac{x-x_{0}}{\delta}\right)$. Let $\vec{\psi} \in C_{0}^{\infty}\left(\mathbb{R}^{n}\right)^{n}$. Setting $v=\zeta_{\delta, x_{0}} \operatorname{div}(1-\Delta)^{-1} \vec{\psi}$ in (6.9) we obtain:

$$
\left|\left\langle Q \eta_{\delta, x_{0}}, v\right\rangle\right| \leq c \delta^{\frac{2}{1+\beta}+\frac{n-2}{2}}
$$




$$
\times\left(\left\|\left(\nabla \zeta_{\delta, x_{0}}\right) \operatorname{div}(1-\Delta)^{-1} \vec{\psi}\right\|_{L^{2}}+\left\|\nabla \operatorname{div}(1-\Delta)^{-1} \vec{\psi}\right\|_{L^{2}}\right) .
$$

It follows, e.g. from the Plancherel theorem,

$$
\left\|\nabla \operatorname{div}(1-\Delta)^{-1} \vec{\psi}\right\|_{L^{2}} \leq c\|\vec{\psi}\|_{L^{2}}
$$

Obviously,

$$
\left|\nabla \zeta_{\delta, x_{0}}(x)\right| \leq c \delta^{-1} \leq c\left|x-x_{0}\right|^{-1}, \quad x \in B_{\delta}\left(x_{0}\right)
$$

For $n \geq 3$, applying the preceding estimates together with Hardy's inequality, we obtain:

$$
\int_{B_{\delta}\left(x_{0}\right)}\left|\nabla \zeta_{\delta, x_{0}}\right|^{2}\left|\operatorname{div}(1-\Delta)^{-1} \vec{\psi}\right|^{2} d x \leq c \int\left|\nabla \operatorname{div}(1-\Delta)^{-1} \vec{\psi}\right|^{2} d x \leq c \|\left.\vec{\psi}\right|_{L^{2}} ^{2}
$$

Thus, in case $n \geq 3$,

$$
\left|\left\langle Q \eta_{\delta, x_{0}}, \operatorname{div}(1-\Delta)^{-1} \vec{\psi}\right\rangle\right| \leq c \delta^{\frac{2}{1+\beta}+\frac{n-2}{2}} \|\left.\vec{\psi}\right|_{L^{2}}
$$

or equivalently,

$$
\left|\int_{\mathbb{R}^{n}} \nabla(1-\Delta)^{-1}\left(\eta_{\delta, x_{0}} Q\right) \cdot \vec{\psi}(x) d x\right| \leq c \delta^{\frac{2}{1+\beta}+\frac{n-2}{2}}\|\vec{\psi}\|_{L^{2}} .
$$

Minimizing over all $\vec{\psi}$, we obtain:

$$
\left\|\nabla(1-\Delta)^{-1}\left(\eta_{\delta, x_{0}} Q\right)\right\|_{L^{2}\left(\mathbb{R}^{n}\right)}^{2} \leq c \delta^{\frac{4}{1+\beta}+n-2} .
$$

Remark 6.1. In the case $n=2$, the preceding estimate fails even for nonnegative $Q$. It can be replaced with a weaker estimate:

$$
\left\|\nabla(1-\Delta)^{-1}\left(\eta_{\delta, x_{0}} Q\right)\right\|_{L^{2}\left(\mathbb{R}^{2}\right)}^{2} \leq c \delta^{\frac{4}{1+\beta}} \log \frac{2}{\delta}, \quad 0<\delta \leq 1 .
$$

Nevertheless, the following localized version still holds for every $n \geq 2$. We set $v=\zeta_{\delta, x_{0}} \operatorname{div}(1-\Delta)^{-1} \vec{\psi}$ in (6.9) where in this case we assume that $\vec{\psi} \in C_{0}^{\infty}\left(B_{\delta}\left(x_{0}\right)\right)^{n}$. Since $\operatorname{supp} \vec{\psi} \subset B_{\delta}\left(x_{0}\right)$, we deduce from (6.11) that, for $x \in B_{\delta}\left(x_{0}\right)$,

$$
\begin{aligned}
& \left|\operatorname{div}(1-\Delta)^{-1} \vec{\psi}(x)\right| \leq c \int_{B_{\delta}\left(x_{0}\right)}\left|\nabla G_{2}(x-y)\right||\vec{\psi}(y)| d y \\
& \leq c \int_{|x-y|<2 \delta}|x-y|^{1-n}|\vec{\psi}(y)| d y,
\end{aligned}
$$

where $G_{\nu}$ is a Bessel kernel of order $\nu>0$. Here we have used the known estimate (see, e.g., $\mathrm{AH}$, Sec. 1.2.5):

$$
\left|\nabla G_{2}(x)\right| \leq c G_{1}(x) \leq c|x|^{1-n}, \quad|x| \leq 1, \quad n \geq 2 .
$$


We now apply the inequality (see $\underline{\mathrm{AH}}]$ ):

$$
\int_{|x-y|<2 \delta}|x-y|^{1-n}|\vec{\psi}(y)| d y \leq c \delta M|\vec{\psi}(x)|,
$$

where $M$ is the Hardy-Littlewood maximal function operator. Hence, by the maximal function inequality,

$$
\int_{B_{\delta}\left(x_{0}\right)}\left|\nabla \zeta_{\delta, x_{0}}(x)\right|^{2}\left|\operatorname{div}(1-\Delta)^{-1} \vec{\psi}(x)\right|^{2} d x \leq c|| M|\vec{\psi}|\left\|_{L^{2}}^{2} \leq c\right\| \vec{\psi}||_{L^{2}}^{2}
$$

This gives:

$$
\left|\int_{B_{\delta}\left(x_{0}\right)} \nabla(1-\Delta)^{-1}\left(\eta_{\delta, x_{0}} Q\right) \cdot \vec{\psi}(x) d x\right| \leq c \delta^{\frac{2}{1+\beta}+\frac{n-2}{2}}\|\vec{\psi}\|_{L^{2}} .
$$

Minimizing over all such $\vec{\psi}$, we arrive at the estimate:

$$
\int_{B_{\delta}\left(x_{0}\right)}\left|\nabla(1-\Delta)^{-1}\left(\eta_{\delta, x_{0}} Q\right)(x)\right|^{2} d x \leq c \delta^{\frac{4}{1+\beta}+n-2} .
$$

We now estimate $\int\left|(1-\Delta)^{-1}\left(\eta_{\delta, x_{0}} Q\right)(x)\right| d x$. Let $\phi$ be a compactly supported function, $\phi \in L^{\infty}\left(\mathbb{R}^{n}\right)$, and set $v=\zeta_{\delta, x_{0}}(1-\Delta)^{-1} \phi$ in (6.9), where $\zeta_{\delta, x_{0}}$ was defined above. Then

$$
\begin{aligned}
\mid\left\langle\eta_{\delta, x_{0}} Q,\right. & \left.(1-\Delta)^{-1} \phi\right\rangle \mid \leq c \delta^{\frac{2}{1+\beta}+\frac{n-2}{2}} \\
& \times\left(\delta^{-2}\left\|(1-\Delta)^{-1} \phi\right\|_{L^{2}\left(B_{\delta}\left(x_{0}\right)\right)}^{2}+\left\|\nabla(1-\Delta)^{-1} \phi\right\|_{L^{2}\left(B_{\delta}\left(x_{0}\right)\right)}^{2}\right)^{\frac{1}{2}} .
\end{aligned}
$$

Notice that $G_{2} \in L^{1}\left(\mathbb{R}^{n}\right), n \geq 2$, and hence

$$
\left\|(1-\Delta)^{-1} \phi\right\|_{L^{\infty}} \leq\|\phi\|_{L^{\infty}}\left\|G_{2}\right\|_{L^{1}}
$$

This gives:

$$
\delta^{-2}\left\|(1-\Delta)^{-1} \phi\right\|_{L^{2}\left(B_{\delta}\left(x_{0}\right)\right)}^{2} \leq c \delta^{n-2}\|\phi\|_{L^{\infty}}^{2} .
$$

Similarly, $\nabla G_{2} \in L^{1}\left(\mathbb{R}^{n}\right), n \geq 2$, and so

$$
\left\|\nabla(1-\Delta)^{-1} \phi\right\|_{L^{\infty}} \leq\|\phi\|_{L^{\infty}}\left\|\nabla G_{2}\right\|_{L^{1}}
$$

which implies:

$$
\left\|\nabla(1-\Delta)^{-1} \phi\right\|_{L^{2}\left(B_{\delta}\left(x_{0}\right)\right)}^{2} \leq c \delta^{n}\|\phi\|_{L^{\infty}}^{2} .
$$

Since $\delta \leq 1$, we can combine the preceding estimates to get:

$$
\left|\left\langle\eta_{\delta, x_{0}} Q,(1-\Delta)^{-1} \phi\right\rangle\right|=\left|\left\langle(1-\Delta)^{-1}\left(\eta_{\delta, x_{0}} Q\right), \phi\right\rangle\right| \leq c \delta^{\frac{2}{1+\beta}+n-2}|| \phi||_{L^{\infty}} .
$$

This yields

$$
\int_{\mathbb{R}^{n}}\left|(1-\Delta)^{-1}\left(\eta_{\delta, x_{0}} Q\right)(x)\right| d x \leq c \delta^{\frac{2}{1+\beta}+n-2}, \quad 0<\delta \leq 1 .
$$


Analogous inequalities hold in the homogeneous case $\delta_{0}=+\infty$. If $n \geq 3$, we set in (6.9) $v=\zeta_{\delta, x_{0}} \operatorname{div} \Delta^{-1} \vec{\psi}$ in (6.9), where $\vec{\psi} \in C_{0}^{\infty}\left(\mathbb{R}^{n}\right)^{n}$. Estimating exactly as above, using Plancherel's theorem and Hardy's inequality, we get:

$$
\int_{\mathbb{R}^{n}}\left|\nabla \Delta^{-1}\left(\eta_{\delta, x_{0}}, Q\right)(x)\right|^{2} d x \leq c \delta^{\frac{4}{1+\beta}+n-2}, \quad 0<\delta<+\infty .
$$

For $n \geq 2$, we assume that $\psi \in C_{0}^{\infty}\left(B_{\delta}\left(x_{0}\right)\right)^{n}$, and notice that, for $x \in B_{\delta}\left(x_{0}\right)$,

$$
\left|\operatorname{div} \Delta^{-1} \vec{\psi}(x)\right| \leq c \int_{|y-x|<2 \delta} \frac{|\vec{\psi}(y)|}{|x-y|^{n-1}} d y \leq c \delta M(|\vec{\psi}|)(x) .
$$

Applying the maximal function inequality, we deduce as in the inhomogeneous case:

$$
\int_{B_{\delta}\left(x_{0}\right)}\left|\nabla \zeta_{\delta, x_{0}}(x)\right|^{2}\left|\operatorname{div} \Delta^{-1} \vec{\psi}(x)\right|^{2} d x+\int_{B_{\delta}\left(x_{0}\right)}\left|\nabla \operatorname{div} \Delta^{-1} \vec{\psi}(x)\right|^{2} d x \leq\left. c\|\vec{\psi}\|\right|_{L^{2}} ^{2}
$$

This yields:

$$
\int_{B_{\delta}\left(x_{0}\right)}\left|\nabla \Delta^{-1}\left(\eta_{\delta, x_{0}}, Q\right)(x)\right|^{2} d x \leq c \delta^{\frac{4}{1+\beta}+n-2}, \quad 0<\delta<+\infty .
$$

We now state the key lemma which gives estimates of the global "antiderivative" of $Q$.

Lemma 6.4. Suppose $Q \in \mathcal{D}^{\prime}\left(\mathbb{R}^{n}\right), n \geq 2$, and $\beta>0$. Suppose that (6.2) holds for every $\delta \in\left(0, \delta_{0}\right)$, where either $\delta_{0}=1$ or $\delta_{0}=+\infty$. Then the following statements hold:

(i) For $\delta_{0}=1$ and $\beta>1$,

$$
\int_{B_{\delta}\left(x_{0}\right)}\left|\nabla(1-\Delta)^{-1} Q\right|^{2} d x \leq c \delta^{\frac{4}{1+\beta}+n-2}, \quad 0<\delta \leq 1
$$

and

$$
\int_{B_{\delta}\left(x_{0}\right)}\left|(1-\Delta)^{-1} Q\right| d x \leq c \delta^{\frac{2}{1+\beta}+n-2}, \quad 0<\delta \leq 1 .
$$

(ii) For $\delta_{0}=1$ and $0<\beta \leq 1$,

$\int_{B_{\delta}\left(x_{0}\right)}\left|\nabla(1-\Delta)^{-1} Q-m_{B_{\delta}\left(x_{0}\right)}\left(\nabla(1-\Delta)^{-1} Q\right)\right|^{2} d x \leq c \delta^{\frac{4}{1+\beta}+n-2}, \quad 0<\delta \leq 1$,

and

$$
\int_{B_{\delta}\left(x_{0}\right)}\left|(1-\Delta)^{-1} Q\right| d x \leq c \delta^{\frac{2}{1+\beta}+n-2}, \quad 0<\delta \leq 1
$$

(iii) For $\delta_{0}=+\infty$ and $\beta>1$,

$$
\int_{B_{\delta}\left(x_{0}\right)}\left|\nabla \Delta^{-1} Q\right|^{2} d x \leq c \delta^{\frac{4}{1+\beta}+n-2}, \quad 0<\delta<+\infty
$$


(iv) For $\delta_{0}=+\infty$ and $0<\beta \leq 1$,

$$
\int_{B_{\delta}\left(x_{0}\right)}\left|\nabla \Delta^{-1} Q-m_{B_{\delta}\left(x_{0}\right)}\left(\nabla \Delta^{-1} Q\right)\right|^{2} d x \leq c \delta^{\frac{4}{1+\beta}+n-2}, \quad 0<\delta<+\infty .
$$

Proof. We fix $x_{0} \in \mathbb{R}^{n}$ and $\delta>0$, and define a special partition of unity $\left\{\psi_{j}\right\}_{j=0}^{+\infty}$ associated with $\delta$ and $x_{0}$. (For the sake of convenience, we will suppress the dependence of $\psi_{j}$ on $\delta, x_{0}$ in our notation used below.)

Let $\eta_{\delta, x_{0}}^{0}(x)=\eta\left(\frac{2\left|x-x_{0}\right|}{\delta}\right)$, where $\eta \in C_{0}^{\infty}\left(\mathbb{R}_{+}\right)$is such that $\eta(x)=1$ if $0 \leq x \leq \frac{1}{2}$ and $\eta(x)=0$ if $x \geq 1$. Next, we pick $\zeta \in C_{0}^{\infty}\left(\mathbb{R}_{+}\right)$so that $\zeta(x)=0$ if $0 \leq x \leq \frac{1}{4}$ or $x \geq 2$, and $\zeta(x)=1$ if $\frac{1}{2} \leq x \leq 1$, and set

$$
\eta_{\delta, x_{0}}^{j}(x)=\zeta\left(\frac{\left|x-x_{0}\right|}{2^{j-2} \delta}\right), \quad j=1,2, \ldots
$$

Letting $\phi_{\delta, x_{0}}(x)=\sum_{j=0}^{+\infty} \eta_{\delta, x_{0}}^{j}(x)$, we see that clearly $\phi_{\delta, x_{0}} \in C^{\infty}\left(\mathbb{R}^{n}\right)$, and $1 \leq \phi_{\delta, x_{0}}(x) \leq 3$. We now set

$$
\psi_{j}(x)=\frac{\eta_{\delta, x_{0}}^{j}(x)}{\phi_{\delta, x_{0}}(x)}, \quad j=0,1, \ldots
$$

We observe that

$$
0 \leq \psi_{j}(x) \leq 1, \quad\left|\nabla \psi_{j}(x)\right| \leq \frac{c}{2^{j} \delta}, \quad j=0,1, \ldots
$$

and

$$
\sum_{j=0}^{+\infty} \psi_{j}(x) \equiv 1, \quad \psi_{j} \in C_{0}^{\infty}\left(B_{2^{j} \delta}\left(x_{0}\right) \backslash B_{2^{j-2} \delta}\left(x_{0}\right)\right), \quad j=1,2, \ldots
$$

We also denote by $\widetilde{\psi}_{j}, j=1,2, \ldots$, a function in $C_{0}^{\infty}\left(B_{2^{j} \delta}\left(x_{0}\right) \backslash B_{2^{j-2} \delta}\left(x_{0}\right)\right)$ with a slightly larger support so that

$$
\widetilde{\psi}_{j}(x) \psi_{j}(x)=\psi_{j}(x), \quad\left|\nabla \widetilde{\psi}_{j}(x)\right| \leq \frac{c}{2^{j} \delta} .
$$

We now prove statement (i). Let $0<\delta \leq 1$ and $\beta>1$. Using (6.23), we obtain:

$$
\left\|\nabla(1-\Delta)^{-1} Q\right\|_{L^{2}\left(B_{\delta}\left(x_{0}\right)\right)} \leq\left\|\sum_{j=0}^{+\infty}\left|\nabla(1-\Delta)^{-1}\left(\psi_{j} Q\right)\right|\right\|_{L^{2}\left(B_{\delta}\left(x_{0}\right)\right)}
$$

Suppose that $j=0,1,2$. Since $\psi_{j} \in C^{\infty}\left(B_{4 \delta}\left(x_{0}\right)\right)$, by Proposition 6.3.

$$
\left\|\nabla(1-\Delta)^{-1}\left(\psi_{j} Q\right)\right\|_{L^{2}\left(B_{\delta}\left(x_{0}\right)\right)} \leq c \delta^{\frac{2}{1+\beta}+\frac{n-2}{2}} .
$$

For $j \geq 3$, notice that

$\left|\nabla(1-\Delta)^{-1}\left(\psi_{j} Q\right)(x)\right|=\left|\nabla(1-\Delta)^{-1}\left(\psi_{j} \widetilde{\psi}_{j} Q\right)(x)\right|=\left|\left\langle\psi_{j} Q, \nabla G_{2}(x-\cdot) \widetilde{\psi}_{j}\right\rangle\right|$ 
Fix $x \in B_{\delta}\left(x_{0}\right)$. Applying (6.9) with $\psi_{j}$ in place of $\eta_{\delta, x_{0}}, \nabla G_{2}(x-\cdot) \widetilde{\psi}_{j}$ in place of $v$, and $2^{j} \delta$ in place of $\delta$ respectively, we obtain:

$$
\begin{aligned}
\left|\nabla(1-\Delta)^{-1}\left(\psi_{j} Q\right)(x)\right| & \leq\left|\left\langle\psi_{j} Q, \nabla(1-\Delta)^{-1} G_{2}(x-\cdot) \widetilde{\psi}_{j}\right\rangle\right| \\
& \leq\left. c\left(2^{j} \delta\right)^{\frac{2}{1+\beta}+\frac{n-2}{2}}|| \nabla\left(\nabla G_{2}(x-\cdot) \widetilde{\psi}_{j}\right)\right|_{L^{2}\left(B_{2^{j} \delta}\left(x_{0}\right)\right)},
\end{aligned}
$$

for $2^{j} \delta \leq 1$. For $2^{j} \delta>1$, a similar estimate follows from (6.10):

$$
\left|\nabla(1-\Delta)^{-1}\left(\psi_{j} Q\right)(x)\right| \leq c\left(2^{j} \delta\right)^{2+\frac{n-2}{2}} \|\left.\nabla\left(\nabla G_{2}(x-\cdot) \widetilde{\psi}_{j}\right)\right|_{L^{2}\left(B_{2^{j} \delta}\left(x_{0}\right)\right)} .
$$

Let $y \in B_{2^{j} \delta}\left(x_{0}\right) \backslash B_{2^{j-2} \delta}\left(x_{0}\right)$. Then $2^{j-3} \delta \leq|y-x| \leq 2^{j+1} \delta, j \geq 3$. Using the preceding inequalities, together with (6.22) and the estimates for the Bessel kernel (see, e.g., $[\mathrm{AH}$, Sec 1.2.5):

$$
\begin{aligned}
& \left|\nabla G_{2}(x-y)\right| \leq \frac{c}{|x-y|^{n-1}}, \quad\left|\nabla \nabla G_{2}(x-y)\right| \leq \frac{c}{|x-y|^{n}}, \quad|x-y| \leq 1, \\
& \left|\nabla G_{2}(x-y)\right| \leq c e^{-|x-y|}, \quad\left|\nabla \nabla G_{2}(x-y)\right| \leq c e^{-|x-y|}, \quad|x-y|>1,
\end{aligned}
$$

we get:

$$
\begin{aligned}
& \left|\nabla_{y}\left(\left(\nabla G_{2}\right)(x-y) \widetilde{\psi}_{j}(y)\right)\right| \leq\left|\nabla G_{2}(x-y)\right|\left|\nabla \widetilde{\psi}_{j}(y)\right| \\
& +\left|\nabla \nabla G_{2}(x-y)\right| \widetilde{\psi}_{j}(y) \leq \frac{c}{\left(2^{j} \delta\right)^{n}}, \quad \text { for } \quad 2^{j} \delta \leq 1
\end{aligned}
$$

and

$$
\left|\nabla_{y}\left(\left(\nabla G_{2}\right)(x-y) \widetilde{\psi}_{j}(y)\right)\right| \leq c e^{-2^{j} \delta}, \quad \text { for } \quad 2^{j} \delta>1
$$

Hence,

$$
\begin{array}{ll}
\left\|\nabla\left(\nabla G_{2}(x-\cdot) \widetilde{\psi}_{j}\right)\right\|_{L^{2}\left(B_{2^{j} \delta}\left(x_{0}\right)\right)} \leq c\left(2^{j} \delta\right)^{-\frac{n}{2}} & \text { if } \quad 2^{j} \delta \leq 1, \\
\left\|\nabla\left(\nabla G_{2}(x-\cdot) \widetilde{\psi}_{j}\right)\right\|_{L^{2}\left(B_{2^{j} \delta}\left(x_{0}\right)\right)} \leq c\left(2^{j} \delta\right)^{\frac{n}{2}} e^{-2^{j} \delta} & \text { if } \quad 2^{j} \delta>1 .
\end{array}
$$

Since $\beta>1$, it follows that $\frac{2}{1+\beta}-1<0$. Thus, for $x \in B_{\delta}\left(x_{0}\right)$, we have a uniform estimate:

$$
\begin{aligned}
& \sum_{j=3}^{+\infty}\left|\nabla(1-\Delta)^{-1}\left(\psi_{j} Q\right)(x)\right| \leq c \sum_{j=3}^{\left[\log _{2} \frac{2}{\delta}\right]}\left(2^{j} \delta\right)^{\frac{2}{1+\beta}+\frac{n-2}{2}}\left(2^{j} \delta\right)^{-\frac{n}{2}} \\
& +c \sum_{j=\left[\log _{2} \frac{2}{\delta}\right]}^{+\infty}\left(2^{j} \delta\right)^{n+1} e^{-2^{j} \delta} \leq c \delta^{\frac{2}{1+\beta}-1} .
\end{aligned}
$$

Hence,

$$
\left\|\sum_{j=3}^{+\infty}\left|\nabla(1-\Delta)^{-1}\left(\psi_{j} Q\right)(x)\right|\right\|_{L^{2}\left(B_{\delta}\left(x_{0}\right)\right)} \leq c \delta^{\frac{2}{1+\beta}+\frac{n-2}{2}}
$$


From the preceding estimates it follows:

$$
\left\|\nabla(1-\Delta)^{-1} Q\right\|_{L^{2}\left(B_{\delta}\left(x_{0}\right)\right)} \leq c \delta^{\frac{2}{1+\beta}+\frac{n-2}{2}} .
$$

A similar argument yields the estimate:

$$
\int_{B_{\delta}\left(x_{0}\right)}\left|(1-\Delta)^{-1} Q\right| d x \leq c \delta^{\frac{2}{1+\beta}+n-2}, \quad 0<\delta \leq 1
$$

We have:

$$
\int_{B_{\delta}\left(x_{0}\right)}\left|(1-\Delta)^{-1} Q\right| d x \leq \sum_{j=0}^{+\infty} \int_{B_{\delta}\left(x_{0}\right)}\left|(1-\Delta)^{-1}\left(\psi_{j} Q\right)\right| d x
$$

By Proposition 6.3.

$$
\int_{B_{\delta}\left(x_{0}\right)}\left|(1-\Delta)^{-1}\left(\psi_{j} Q\right)\right| d x \leq c \delta^{\frac{2}{1+\beta}+n-2}, \quad 0<\delta \leq 1,
$$

for $j=0,1,2$. If $j \geq 3$ and $x \in B_{\delta}\left(x_{0}\right)$, we estimate using Proposition 3.3 as above:

$$
\begin{aligned}
\left|(1-\Delta)^{-1}\left(\psi_{j} Q\right)(x)\right| & \leq\left|\left\langle\psi_{j} Q, G_{2}(x-\cdot) \widetilde{\psi}_{j}\right\rangle\right| \\
& \leq c\left(2^{j} \delta\right)^{\frac{2}{1+\beta}+\frac{n-2}{2}}\left\|\nabla\left(G_{2}(x-\cdot) \widetilde{\psi}_{j}\right)\right\|_{L^{2}},
\end{aligned}
$$

if $2^{j} \delta \leq 1$. For $2^{j} \delta>1$

$$
\left|(1-\Delta)^{-1}\left(\psi_{j} Q\right)(x)\right| \leq c\left(2^{j} \delta\right)^{2+\frac{n-2}{2}}\left\|\nabla\left(G_{2}(x-\cdot) \widetilde{\psi}_{j}\right)\right\|_{L^{2}} .
$$

Suppose $n \geq 3$. For $y \in B_{2^{j} \delta}\left(x_{0}\right) \backslash B_{2^{j-2} \delta}\left(x_{0}\right)$, we have $2^{j-3} \delta \leq|y-x| \leq$ $2^{j+1} \delta, j \geq 3$. Hence, for $2^{j} \delta \leq 1$ and $|x-y| \leq 1$,

$$
\left|\nabla\left(G_{2}(x-y) \widetilde{\psi}_{j}(y)\right)\right| \leq\left|\nabla G_{2}(x-y)\right| \widetilde{\psi}_{j}(y)+G_{2}(x-y)\left|\nabla \widetilde{\psi}_{j}(y)\right| \leq c\left(2^{j} \delta\right)^{1-n} \text {. }
$$

Similarly, for $2^{j} \delta>1$ and $|x-y|>1$,

$$
\left|\nabla\left(G_{2}(x-y) \widetilde{\psi}_{j}(y)\right)\right| \leq c e^{-2^{j} \delta}
$$

Thus,

$$
\begin{array}{ll}
\left\|\nabla\left(G_{2}(x-\cdot) \widetilde{\psi}_{j}\right)\right\|_{L^{2}} \leq c\left(2^{j} \delta\right)^{1-\frac{n}{2}}, & \text { if } \quad 2^{j} \delta \leq 1, \\
\left\|\nabla\left(G_{2}(x-\cdot) \widetilde{\psi}_{j}\right)\right\|_{L^{2}} \leq c\left(2^{j} \delta\right)^{\frac{n}{2}} e^{-2^{j} \delta}, & \text { if } \quad 2^{j} \delta>1 .
\end{array}
$$

It follows:

$$
\sum_{j=3}^{+\infty}\left|(1-\Delta)^{-1}\left(\psi_{j} Q\right)(x)\right| \leq c \sum_{j=3}^{\log \frac{2}{\delta}}\left(2^{j} \delta\right)^{\frac{2}{1+\beta}}+\sum_{j>\log \frac{2}{\delta}}\left(2^{j} \delta\right)^{n+1} e^{-2^{j} \delta} \leq C,
$$

where $C$ does not depend on $\delta$. 
A similar uniform estimate holds in the case $n=2$ as well, since $G_{2}(x-y) \leq$ $c \log \frac{2}{2^{j} \delta}$ if $2^{j} \delta \leq|x-y| \leq 2^{j+2} \delta$ and $2^{j} \delta \leq 1$, and hence

$$
\sum_{j=3}^{+\infty}\left|(1-\Delta)^{-1}\left(\psi_{j} Q\right)(x)\right| \leq c \sum_{j=3}^{\log \frac{2}{\delta}} \log \frac{2}{2^{j} \delta}\left(2^{j} \delta\right)^{\frac{2}{1+\beta}}+\sum_{j>\log \frac{2}{\delta}}\left(2^{j} \delta\right)^{3} e^{-2^{j} \delta} \leq C,
$$

where $C$ does not depend on $\delta$.

Combining the preceding estimates we obtain:

$$
\int_{B_{\delta}\left(x_{0}\right)}\left|(1-\Delta)^{-1} Q\right| d x \leq c \delta^{\frac{2}{1+\beta}+n-2}, \quad 0<\delta \leq 1
$$

which proves statement (i) of Lemma 6.4.

To prove statement (ii), we modify the above argument as follows. For $j=0,1,2$, it follows from Proposition 3.4 that

$$
\int_{B_{\delta}\left(x_{0}\right)}\left|\nabla(1-\Delta)^{-1}\left(\psi_{j} Q\right)\right|^{2} d x \leq c \delta^{\frac{4}{1+\beta}+n-2}, \quad 0<\delta \leq 1 .
$$

Obviously, this gives as well the estimate:

$$
\int_{B_{\delta}\left(x_{0}\right)}\left|\nabla(1-\Delta)^{-1}\left(\psi_{j} Q\right)-m_{B_{\delta}\left(x_{0}\right)}\left(\nabla(1-\Delta)^{-1}\left(\psi_{j} Q\right)\right)\right|^{2} d x \leq c \delta^{\frac{4}{1+\beta}+n-2},
$$

where $0<\delta \leq 1$.

For $j=3,4, \ldots$ and $x, x^{\prime} \in B_{\delta}\left(x_{0}\right)$, we deduce the following uniform estimate:

$$
\begin{aligned}
& \left|\nabla(1-\Delta)^{-1}\left(\psi_{j} Q\right)(x)-\nabla(1-\Delta)^{-1}\left(\psi_{j} Q\right)\left(x^{\prime}\right)\right| \\
& \leq\left|\left\langle\psi_{j} Q,\left(\nabla G_{2}(x-\cdot)-\nabla G_{2}\left(x^{\prime}-\cdot\right)\right) \vec{\psi}_{j}\right\rangle\right| \\
& \leq c\left(2^{j} \delta\right)^{\frac{2}{1+\beta}+\frac{n-2}{2}}|| \nabla\left(\left(\nabla G_{2}(x-\cdot)-\nabla G_{2}\left(x^{\prime}-\cdot\right)\right) \vec{\psi}_{j}\right) \|_{L^{2}},
\end{aligned}
$$

if $2^{j} \delta \leq 1$; for $2^{j} \delta>1$, there is a similar estimate with $\beta=0$ on the right-hand side.

The rest of the proof is similar to the case $\beta>1$. For $x, x^{\prime} \in B_{\delta}\left(x_{0}\right)$ and $y \in B_{2^{j} \delta}\left(x_{0}\right) \backslash B_{2^{j-2} \delta}\left(x_{0}\right)$, in the case $2^{j} \delta \leq 1$, we use the estimates

$$
\begin{aligned}
& \left|\nabla G_{2}(x-y)-\nabla G_{2}\left(x^{\prime}-y\right)\right| \leq c \delta\left(2^{j} \delta\right)^{-n}, \\
& \left|\nabla \nabla G_{2}(x-y)-\nabla \nabla G_{2}\left(x^{\prime}-y\right)\right| \leq c \delta\left(2^{j} \delta\right)^{-n-1},
\end{aligned}
$$

together with similar estimates:

$$
\begin{aligned}
& \left|\nabla G_{2}(x-y)-\nabla G_{2}\left(x^{\prime}-y\right)\right| \leq c \delta e^{-2^{j} \delta}, \\
& \left|\nabla \nabla G_{2}(x-y)-\nabla \nabla G_{2}\left(x^{\prime}-y\right)\right| \leq c \delta e^{-2^{j} \delta},
\end{aligned}
$$


in the case $2^{j} \delta>1$. From this it follows exactly as in the proof of statement (i):

$$
\begin{array}{ll}
\left|\nabla(1-\Delta)^{-1}\left(\psi_{j} Q\right)(x)-\nabla(1-\Delta)^{-1}\left(\psi_{j} Q\right)\left(x^{\prime}\right)\right| \leq c \delta\left(2^{j} \delta\right)^{-2+\frac{2}{1+\beta}}, & 2^{j} \delta \leq 1, \\
\left|\nabla(1-\Delta)^{-1}\left(\psi_{j} Q\right)(x)-\nabla(1-\Delta)^{-1}\left(\psi_{j} Q\right)\left(x^{\prime}\right)\right| \leq c \delta e^{-2^{j} \delta}, & 2^{j} \delta>1 .
\end{array}
$$

Hence, for every $\beta>0$, and $x, x^{\prime} \in B_{\delta}\left(x_{0}\right)$ :

$$
\sum_{j \geq 3}\left|\nabla(1-\Delta)^{-1}\left(\psi_{j} Q\right)(x)-\nabla(1-\Delta)^{-1}\left(\psi_{j} Q\right)\left(x^{\prime}\right)\right| \leq c \delta^{-1+\frac{2}{1+\beta}}
$$

Consequently,

$$
\int_{B_{\delta}\left(x_{0}\right)}\left|\nabla(1-\Delta)^{-1} Q-m_{B_{\delta}\left(x_{0}\right)}\left(\nabla(1-\Delta)^{-1} Q\right)\right|^{2} d x \leq c \delta^{n-2+\frac{4}{1+\beta}}, \quad 0<\delta \leq 1 .
$$

Notice that a lower term estimate

$$
\int_{B_{\delta}\left(x_{0}\right)}\left|(1-\Delta)^{-1} Q\right| d x \leq c \delta^{n-2+\frac{2}{1+\beta}}, \quad 0<\delta \leq 1
$$

was proved above for every $\beta>0$. This completes the proof of statement (ii) of Lemma 6.4.

The proofs of statements (iii) and (iv) in the homogeneous case are similar but simpler, and follow by using analogous estimates for the derivatives of Riesz kernels in place of Bessel kernels.

Statement (ii) of Theorem 6.1 now follows from Propositions 6.2 and 6.3 . The proof of Theorem 6.1 is complete.

Remark 6.2. Estimates similar to those used in the proof of Lemma 6.4 in the inhomogeneous case $\delta_{0}=1$, give additionally that (6.2) implies:

$$
\int_{B_{1}\left(x_{0}\right)}\left|\nabla(1-\Delta)^{-1} Q\right|^{2} d x \leq A
$$

for all $\beta>0$, where the constant $A$ does not depend on $x_{0} \in \mathbb{R}^{n}$. In particular, for $\beta=1$, this inequality together with the mean oscillation condition proved in Lemma 6.4 (ii), gives that $\nabla(1-\Delta)^{-1} Q \in \operatorname{bmo}\left(\mathbb{R}^{n}\right)$. For $0<\beta<1$, similar estimates yield:

$$
\left\|\nabla(1-\Delta)^{-1} Q\right\|_{L^{\infty}\left(R^{n}\right)} \leq B
$$

Together with the mean oscillation condition of Lemma 6.4 (ii) this gives: $\nabla(1-\Delta)^{-1} Q \in \operatorname{Lip}_{\frac{1-\beta}{1+\beta}}\left(\mathbb{R}^{n}\right)$.

The following theorem, which also uses Lemma 6.4 in the necessity part, treats the case $0<\beta \leq 1$. 
Theorem 6.5. Let $Q \in \mathcal{D}^{\prime}\left(\mathbb{R}^{n}\right), n \geq 2$, and $0<\beta \leq 1$. Then the following statements hold.

(i) Suppose that there exist $\vec{\Gamma} \in L_{\text {loc }}^{2}\left(\mathbb{R}^{n}\right)^{n}$ and $\gamma \in L_{\text {loc }}^{2}$ such that

$$
Q=\operatorname{div} \vec{\Gamma}+\gamma,
$$

where $\vec{\Gamma}$ and $\gamma$ satisfy respectively the inequalities:

$$
\begin{aligned}
& \int_{B_{\delta}\left(x_{0}\right)}\left|\vec{\Gamma}(x)-m_{B_{\delta}\left(x_{0}\right)}(\vec{\Gamma})\right|^{2} d x \leq C_{1} \delta^{n-2+\frac{4}{1+\beta}}, \quad 0<\delta<\delta_{0}, \\
& \int_{B_{\delta}\left(x_{0}\right)}|\gamma(x)| d x \leq C_{2} \delta^{n-2+\frac{2}{1+\beta}}, \quad 0<\delta<\delta_{0},
\end{aligned}
$$

with the constants $C_{1}, C_{2}$ which do not depend on $x_{0}$ and $\delta$. Then there exist $c>0$ and $\epsilon_{0}>0$ such that (6.1) holds for every $\epsilon \in\left(0, \epsilon_{0}\right)$.

(ii) Conversely, suppose (6.1) holds for every $\epsilon \in\left(0, \epsilon_{0}\right)$. Then $Q$ can be represented in the form (6.24) where $\vec{\Gamma} \in L_{\mathrm{loc}}^{2}\left(\mathbb{R}^{n}\right)^{n}, \gamma \in L_{\mathrm{loc}}^{2}\left(\mathbb{R}^{n}\right)$ satisfy respectively conditions (6.25) and (6.26).

(iii) If $\epsilon_{0}=1$, then one can set

$$
\vec{\Gamma}=-\nabla(1-\Delta)^{-1} Q, \quad \gamma=(1-\Delta)^{-1} Q,
$$

in (6.24), where $\delta_{0}=1$ in (6.25), (6.26).

If $\epsilon_{0}=+\infty$, then one can set

$$
\vec{\Gamma}=\nabla \Delta^{-1} Q, \quad \gamma=0,
$$

in (6.24), where $\delta_{0}=+\infty$ in (6.25), (6.26).

Proof. We need only to prove statement (i) since (ii) and (iii) follow from Lemma 6.4. Suppose $0<\beta \leq 1$ and $Q$ is represented in the form (6.24) so that (6.25), 6.26) hold. The proof is very similar to the case $\beta>1$ except for the estimate

$$
|\langle Q u, u\rangle|=|\langle\vec{\Gamma} u, \nabla u\rangle| \leq c\left\|\left.\nabla u\right|_{L^{2}} ^{\frac{2 \beta}{1+\beta}}\right\| u \|_{L^{2}}^{\frac{2}{1+\beta}}, \quad u \in C_{0}^{\infty}\left(\mathbb{R}^{n}\right),
$$

or, equivalently by Corollary 3.3

$$
|\langle Q u, u\rangle|=|\langle\vec{\Gamma} u, \nabla u\rangle| \leq c \delta^{\frac{2}{1+\beta}}\|\nabla u\|_{L^{2}}^{2} \quad u \in C_{0}^{\infty}\left(B_{\delta}\left(x_{0}\right)\right) .
$$

We use known characterizations of the Morrey-Campanato spaces. In particular, for $0<\beta<1$, condition (6.25) is equivalent to the condition $\vec{\Gamma} \in$ $\operatorname{Lip}_{\alpha}\left(\mathbb{R}^{n}\right)$, where $\alpha=\frac{1-\beta}{1+\beta}$. In other words,

$$
\left|\vec{\Gamma}(x)-\vec{\Gamma}\left(x^{\prime}\right)\right| \leq C\left|x-x^{\prime}\right|^{\alpha}, \quad\left|x-x^{\prime}\right| \leq \delta_{0} .
$$

Then, for $u \in C_{0}^{\infty}\left(B_{\delta}\left(x_{0}\right)\right)$,

$$
|\langle\vec{\Gamma} u, \nabla u\rangle|=\left|\int_{B_{\delta}\left(x_{0}\right)}\left(\vec{\Gamma}-m_{B_{\delta}\left(x_{0}\right)}(\vec{\Gamma})\right) \cdot \nabla u u d x\right| \leq c \delta^{\frac{1-\beta}{1+\beta}} \int_{B_{\delta}\left(x_{0}\right)}|\nabla u||u| d x .
$$


Using Schwarz's inequality and the estimate

$$
\int_{B_{\delta}\left(x_{0}\right)}|u(x)|^{p} d x \leq c \delta^{p} \int_{B_{\delta}\left(x_{0}\right)}|\nabla u(x)|^{p} d x, \quad u \in C_{0}^{\infty}\left(B_{\delta}\left(x_{0}\right)\right),
$$

with $p=2$, we obtain (6.29).

In the case $\beta=1$, we have $\vec{\Gamma} \in \operatorname{BMO}\left(\mathbb{R}^{n}\right)$ if $\delta_{0}=+\infty$, or $\vec{\Gamma} \in \operatorname{bmo}\left(\mathbb{R}^{n}\right)$ if $\delta_{0}=1$ respectively. To prove (6.29) we apply Hölder's inequality with exponents $\frac{2 p}{p-2}, 2$, and $p$ so that $\frac{2 p}{p-2}+\frac{1}{2}+\frac{1}{p}=1$, where $2<p<\frac{n}{n-2}$, and $n \geq 2$ :

$$
\begin{aligned}
& |\langle\vec{\Gamma} u, \nabla u\rangle|=\left|\int_{B_{\delta}\left(x_{0}\right)}\left(\vec{\Gamma}-m_{B_{\delta}\left(x_{0}\right)}(\vec{\Gamma})\right) \cdot \nabla u u d x\right| \\
& \leq c|| \vec{\Gamma}-\left.\left.m_{B_{\delta}\left(x_{0}\right)}(\vec{\Gamma})\right|_{L^{\frac{2 p}{p-2}\left(B_{\delta}\left(x_{0}\right)\right)}}|| \nabla u\right|_{L^{2}\left(B_{\delta}\left(x_{0}\right)\right)} \|\left. u\right|_{L^{p}\left(B_{\delta}\left(x_{0}\right)\right)} .
\end{aligned}
$$

It is well-known (and is a consequence of the John-Nirenberg inequality) that $\vec{\Gamma} \in \operatorname{BMO}\left(\mathbb{R}^{n}\right)$ if $\delta_{0}=+\infty$, and respectively $\vec{\Gamma} \in \operatorname{bmo}\left(\mathbb{R}^{n}\right)$ if $\delta_{0}=1$, yield

$$
\int_{B_{\delta}\left(x_{0}\right)}\left|\vec{\Gamma}-m_{B_{\delta}\left(x_{0}\right)}(\vec{\Gamma})\right|^{p} d x \leq c \delta^{n}, \quad 0<\delta \leq \delta_{0}
$$

for any $1 \leq p<\infty$. Applying this inequality together with (6.30), we obtain (6.29). This completes the proof of Theorem 6.5.

It is easy to see that in the case $\beta=1$ and $\epsilon_{0}=+\infty$, the sufficiency part of Theorem 6.5 is equivalent to the inequality

$$
|\langle\vec{\Gamma} u, \nabla u\rangle| \leq c\|\vec{\Gamma}\|_{\mathrm{BMO}\left(\mathbb{R}^{n}\right)}\left\|\left.u\right|_{L^{2}\left(\mathbb{R}^{n}\right)}|| \nabla u\right\|_{L^{2}\left(\mathbb{R}^{n}\right)}, \quad \forall u \in C_{0}^{\infty}\left(\mathbb{R}^{n}\right) .
$$

By duality, the preceding inequality yields:

$$
\|u \nabla u\|_{\mathcal{H}^{1}\left(\mathbb{R}^{n}\right)} \leq c\|u\|_{L^{2}\left(\mathbb{R}^{n}\right)}\|\nabla u\|_{L^{2}\left(\mathbb{R}^{n}\right)}, \quad \forall u \in C_{0}^{\infty}\left(\mathbb{R}^{n}\right) .
$$

As an immediate consequence, we obtain the vector-valued quadratic form inequality mentioned in Sec. 2 .

$$
\|(\vec{u} \cdot \nabla) \vec{u}\|_{\mathcal{H}^{1}\left(\mathbb{R}^{n}\right)} \leq c\|\vec{u}\|_{L^{2}\left(\mathbb{R}^{n}\right)}|| \nabla \vec{u} \|_{L^{2}\left(\mathbb{R}^{n}\right)}, \quad \operatorname{div} \vec{u}=\overrightarrow{0}
$$

for all $\vec{u} \in C_{0}^{\infty}\left(\mathbb{R}^{n}\right)^{n}$.

Both of the preceding inequalities are corollaries of the following nonhomogeneous version of the div-curl lemma in the case $p=2$. For the sake of completeness, we give a proof which is similar to that in [CLMS] where it is assumed additionally that $\operatorname{div} \vec{u}=\overrightarrow{0}$.

Lemma 6.6. Let $1<p<+\infty$ and $\frac{1}{p}+\frac{1}{p^{\prime}}=1$. Let $\vec{u} \in W^{1, p}\left(\mathbb{R}^{n}\right)^{n}$ and $v \in W^{1, p^{\prime}}\left(\mathbb{R}^{n}\right)$. Then

$$
\begin{aligned}
\|\operatorname{div}(\vec{u} v)\|_{\mathcal{H}^{1}\left(\mathbb{R}^{n}\right)} & \leq c\left(\|\vec{u}\|_{L^{p}\left(\mathbb{R}^{n}\right)}|| \nabla v \|_{L^{p^{\prime}\left(\mathbb{R}^{n}\right)}}\right. \\
& \left.+\|\operatorname{div} \vec{u}\|_{L^{p}\left(\mathbb{R}^{n}\right)}\|v\|_{L^{p^{\prime}\left(\mathbb{R}^{n}\right)}}\right)
\end{aligned}
$$


where $c$ does not depend on $\vec{u}$ and $v$.

Proof. Without loss of generality we may assume that $\vec{u} \in C_{0}^{\infty}\left(\mathbb{R}^{n}\right)^{n}$ and $v \in C_{0}^{\infty}\left(\mathbb{R}^{n}\right)$. Fix $\phi \in C_{0}^{\infty}\left(B_{1}(0)\right)$ and set $\phi_{\delta}=\delta^{-n} \phi\left(\frac{x}{\delta}\right), \delta>0$. Let $B=B_{\delta}(x)$, $x \in \mathbb{R}^{n}$. Then

$$
\|\operatorname{div}(\vec{u} v)\|_{\mathcal{H}^{1}\left(\mathbb{R}^{n}\right)} \asymp\left\|\sup _{\delta>0}\left|\operatorname{div}(\vec{u} v) \star \phi_{\delta}\right|\right\|_{L^{1}\left(\mathbb{R}^{n}\right)} .
$$

Note that

$$
\begin{aligned}
\operatorname{div}(\vec{u} v) \star \phi_{\delta}(x) & =-\delta^{-1-n} \int_{B} \nabla \phi\left(\frac{x-y}{\delta}\right) \cdot \vec{u}(y)\left(v(y)-m_{B}(v)\right) d y \\
& +\delta^{-n} m_{B}(v) \int_{B} \phi_{\delta}(x-y) \operatorname{div} \vec{u}(y) d y
\end{aligned}
$$

As in the proof of Lemma II.1 in [CLMS], pick $\alpha, \beta$ so that $\frac{1}{\alpha}-\frac{1}{n}=1-\frac{1}{\beta}$, and $1 \leq \alpha<p, 1<\beta<p^{\prime}$. By Hölder's inequality,

$$
\begin{aligned}
\left|\operatorname{div}(\vec{u} v) \star \phi_{\delta}(x)\right| & \leq c\left(\delta^{-n} \int_{B}|\vec{u}(y)|^{\beta} d y\right)^{\frac{1}{\beta}}\left(\delta^{-n-\beta^{\prime}} \int_{B}\left|v(y)-m_{B}(v)\right|^{\beta^{\prime}} d y\right)^{\frac{1}{\beta^{\prime}}} \\
& +c\left|m_{B}(v)\right| \delta^{-n} \int_{B}|\operatorname{div} \vec{u}(y)| d y
\end{aligned}
$$

By Poincaré's inequality,

$$
\left(\delta^{-n-\beta^{\prime}} \int_{B}\left|v(y)-m_{B}(v)\right|^{\beta^{\prime}} d y\right)^{\frac{1}{\beta^{\prime}}} \leq c\left(\delta^{-n} \int_{B}|\nabla v|^{\alpha}\right)^{\frac{1}{\alpha}} \leq c\left(M|\nabla v|^{\alpha}(x)\right)^{\frac{1}{\alpha}},
$$

where $M$ is the Hardy-Littlewood maximal operator. Also, clearly,

$$
\left|m_{B}(v)\right| \delta^{-n} \int_{B}|\operatorname{div} \vec{u}(y)| d y \leq M v(x) M(\operatorname{div} \vec{u})(x) .
$$

Combining these estimates, we get:

$$
\left|\operatorname{div}(\vec{u} v) \star \phi_{\delta}(x)\right| \leq c\left(M|\vec{u}|^{\beta}(x)\right)^{\frac{1}{\beta}}\left(M|\nabla v|^{\alpha}(x)\right)^{\frac{1}{\alpha}}+c M v(x) M(\operatorname{div} \vec{u})(x) .
$$

Applying Hölder's inequality together with the maximal inequality, we obtain (6.31).

The following corollary is an immediate consequence of Theorem 6.5, Remark 6.2, and the characterizations of the Morrey-Campanato spaces mentioned above.

Corollary 6.7. (i) Under the assumptions of Theorem [6.5, in the case $\beta=1$, condition (6.25) holds if and only if $\vec{\Gamma} \in \mathrm{BMO}\left(\mathbb{R}^{n}\right)$ if $\delta_{0}=+\infty$, and respectively $\vec{\Gamma} \in \operatorname{bmo}\left(\mathbb{R}^{n}\right)$ if $\delta_{0}=1$. 
(ii) Similarly, in the case $0<\beta<1$, condition (6.25) holds if and only if $\vec{\Gamma}$ is in the homogeneous Lipschitz space $\operatorname{Lip}_{\frac{1-\beta}{1+\beta}}\left(\mathbb{R}^{n}\right)$ if $\delta_{0}=+\infty$, and respectively in the corresponding inhomogeneous space $\operatorname{Lip}_{\frac{1-\beta}{1+\beta}}\left(\mathbb{R}^{n}\right)$ if $\delta_{0}=1$.

Remark 6.3. In the case $0<\beta \leq 1$, Theorem 6.5 and Corollary 6.7 can be restated directly in terms of conditions imposed on $Q$ using the scale of Triebel-Lizorkin spaces $F_{\alpha}^{p, q}$ (or Besov spaces $B_{\alpha}^{p, q}$ if $0<\beta<1$ ) with negative index $\alpha$ (see, e.g., Tri]).

More precisely, it is easy to see that, for $\beta=1$, conditions (6.25) and (6.26) in the homogeneous case are equivalent to $Q \in \dot{F}_{\infty}^{-1,2}\left(\mathbb{R}^{n}\right)=\mathrm{BMO}_{-1}\left(\mathbb{R}^{n}\right)$. For $0<\beta<1$, an equivalent condition on $Q$ is given by: $Q \in \dot{F}_{\infty}^{-\frac{2 \beta}{1+\beta}, \infty}\left(\mathbb{R}^{n}\right)=$ $\dot{B}_{\infty}^{-\frac{2 \beta}{1+\beta}, \infty}\left(\mathbb{R}^{n}\right)$. Similarly, in the inhomogeneous case we have $Q \in F_{\infty}^{-1,2}\left(\mathbb{R}^{n}\right)$ for $\beta=1$ and $Q \in B_{\infty}^{-\frac{2 \beta}{1+\beta}, \infty}\left(\mathbb{R}^{n}\right)$ respectively.

Finally, we are in a position to characterize multiplicative quadratic form inequalities of Nash's type:

$$
|\langle Q u, u\rangle| \leq C\|\nabla u\|_{L^{2}\left(\mathbb{R}^{n}\right)}^{2 p}\|u\|_{L^{1}\left(\mathbb{R}^{n}\right)}^{2(1-p)}, \quad \forall u \in C_{0}^{\infty}\left(\mathbb{R}^{n}\right),
$$

where $p \in(0,1)$.

Corollary 6.8. Suppose $Q \in D^{\prime}\left(\mathbb{R}^{n}\right), n \geq 2$, and $0<p<1$. Let $p_{*}=\frac{n}{n+2}$ and $p^{*}=\frac{n+1}{n+2}$. Then (6.32) holds if anyone of the following conditions is valid:

(a) $0<p<p_{*}$ and $Q=0$.

(b) $p=p_{*}$ and $Q \in L^{\infty}\left(\mathbb{R}^{n}\right)$.

(c) $p_{*}<p<p^{*}$ and $Q=\operatorname{div} \vec{\Gamma}$, where $\vec{\Gamma} \in \operatorname{Lip}_{n+1-p(n+2)}\left(\mathbb{R}^{n}\right)$.

(d) $p=p^{*}$ and $Q=\operatorname{div} \vec{\Gamma}$, where $\vec{\Gamma} \in \operatorname{BMO}\left(\mathbb{R}^{n}\right)$.

(e) $p^{*}<p<1$ and $Q=\operatorname{div} \vec{\Gamma}$, where $\vec{\Gamma} \in \mathcal{L}^{2, \lambda}\left(\mathbb{R}^{n}\right)$, and $\lambda=3 n+2-2 p(n+2)$.

Conversely, if (6.32) holds then conditions (a)-(e) are valid where one may set $\vec{\Gamma}=\nabla \Delta^{-1} Q$.

Corollary 6.8 follows by combining Theorem 6.1] and Theorem 6.5 with Corollary 3.3 and Remark 3.1.

\section{REFERENCES}

[AH] D. R. Adams and L. I. Hedberg, Function Spaces and Potential Theory, SpringerVerlag, Berlin-Heidelberg-New York, 1996.

[Agm] S. Agmon, On positive solutions of elliptic equations with periodic coefficients in $\mathbb{R}^{n}$, spectral results and extensions to elliptic operators on Riemannian manifolds, Differential Equations (Birmingham, Ala., 1983), 7-17. North-Holland Math. Stud. 92, North-Holland, Amsterdam, 1984.

[Agr] M. S. Agranovich, On series in root vectors of operators defined by forms with a selfadjoint principal part, Funct. Anal Appl., 28 (1994), 151-167. 
[AiSi] M. Aizenman and B. Simon, Brownian motion and Harnack inequality for Schrödinger operators, Comm. Pure Appl. Math., 35 (1982), 209-273.

[BiSo] M. S. Birman and M. Z. Solomjak, Schrödinger operator. Estimates for number of bound states as function-theoretical problem, Amer. Math. Soc. Transl., Ser. 2, 150, 1-54. Amer. Math. Soc., Providence, RI, 1992.

[BSh] F. A. Berezin and M. A. Shubin, The Schrödinger Equation, Kluwer Academic Publishers, Dordrecht, 1991.

[BoBr] J. Bourgain and $\mathrm{H}$. Brezis, On the equation $\operatorname{div} Y=f$ and application to control of phases, J. Amer. Math. Soc., 16 (2003), 393-426.

[BrK] H. Brezis and T. Kato, Remarks on the Schrödinger operator with singular complex potentials, J. Math. Pures Appl., 58 (1979), 137-151.

[COV] C. Cascante, J. M. Ortega, and I. E. Verbitsky, Nonlinear potentials and two weight trace inequalities for general dyadic and radial kernels, preprint math.FA/0309286 to appear in Indiana Univ. Math. J.

[ChWW] S.-Y. A. Chang, J. M. Wilson, and T. H. Wolff, Some weighted norm inequalities concerning the Schrödinger operators, Comment. Math. Helv. 60 (1985), 217-246.

[CZh] K. L. Chung and Z. Zhao, From Brownian motion to Schrödinger's equation, Springer-Verlag, Berlin-Heidelberg-New York, 1995.

[CLMS] R. Coifman, P. L. Lions, Y. Meyer and S. Semmes, Compensated compactness and Hardy spaces, J. Math. Pures Appl., 72 (1993), 247-286.

[Co] P. Constantin, Remarks on the Navier-Stokes equations, New Perspectives in Turbulence, 229-261. Springer-Verlag, New York, 1991.

[Dav1] E. B. Davies, $L^{p}$ spectral theory of higher order elliptic differential operators, Bull. London Math. Soc. 29 (1997), 513-546.

[Dav2] E. B. Davies, Non-self-adjoint differential operators, Bull. London Math. Soc. 34 (2002), 513-532.

[EE] D. E. Edmunds and W. D. Evans, Spectral Theory and Differential Operators, Clarendon Press, Oxford, 1987.

[Fef] C. Fefferman, The uncertainty principle, Bull. Amer. Math. Soc. 9 (1983), 129206.

[GT] D. Gilbarg and N. S. Trudinger, Elliptic Partial Differential Equations of Second Order, Reprint of the 1998 edition. Classics in Mathematics, Springer-Verlag, Berlin-Heidelberg-New York, 2001.

[Gr1] E. Grinshpun, On spectral properties of Schrödinger-type operator with complex potential, Oper. Theory: Adv. Appl. 87 (1996), 164-176.

[Gr2] E. Grinshpun, Asymptotics of spectrum under infinitesimally form-bounded perturbation, Integral Eqs. Oper. Theory 19 (1994), 240-250.

[HMV] K. Hansson, V. G. Maz'ya, and I. E. Verbitsky, Criteria of solvability for multidimensional Riccati's equations, Arkiv för Matem. 37 (1999), 87-120.

[HW] L. I. Hedberg and Th. H. Wolff, Thin sets in nonlinear potential theory, Ann. Inst. Fourier (Grenoble), 33 (1983), 161-187.

[Ka1] T. Kato, Schrödinger operators with singular potentials, Israel J. Math. 13 (1972), $135-148$.

[Ka2] T. Kato, Perturbation Theory for Linear Operators, Springer-Verlag, BerlinHeidelberg-New York, 1995.

[KS] R. Kerman and E. Sawyer, The trace inequality and eigenvalue estimates for Schrödinger operators, Ann. Inst. Fourier, Grenoble 36 (1987), 207-228. 
[Ko] P. Koosis, The Logarithmic Integral I, Cambridge Studies in Advanced Mathematics 12, Cambridge, Cambridge University Press, 1998.

[LL] E. H. Lieb and M. Loss, Analysis, Second Edition, Amer. Math. Soc., Providence, RI, 2001.

[LPS] V.A. Liskevich, M.A. Perelmuter, and Yu. A. Semenov, Form-bounded perturbations of generators of sub-Markovian semigroups, Acta Appl. Math. 44 (1996), 353-377.

[MM1] A. S. Marcus and V. I. Matsaev, Operators associated with sesquilinear forms and spectral asymptotics, Mat. Issled. 61 (1981), 86-103.

[MM2] A. S. Marcus and V. I. Matsaev, Comparison theorems for spectra of linear operators and spectral asymptotics, Trans. Moscow Math. Soc. 45 (1984), 139-187.

[M1] V. G. Maz'ya, On the theory of the n-dimensional Schrödinger operator, Izv. Akad. Nauk SSSR, ser. Matem. 28 (1964), 1145-1172.

[M2] V. G. Maz'ya, Sobolev Spaces, Springer-Verlag, Berlin-Heidelberg-New York, 1985.

[MV1] V. G. Maz'ya and I. E. Verbitsky, Capacitary estimates for fractional integrals, with applications to partial differential equations and Sobolev multipliers, Arkiv för Matem. 33 (1995), 81-115.

[MV2] V. G. Maz'ya and I. E. Verbitsky, The Schrödinger operator on the energy space: boundedness and compactness criteria, Acta Math. 188 (2002), 263-302.

[MV3] V. G. Maz'ya and I. E. Verbitsky, Boundedness and compactness criteria for the one-dimensional Schrödinger operator. In: Function Spaces, Interpolation Theory and Related Topics. Proc. Jaak Peetre Conf., Lund, Sweden, August 17-22, 2000. Eds. M. Cwikel, A. Kufner, G. Sparr. De Gruyter, Berlin, 2002, 369-382.

[MV4] V. G. Maz'ya and I. E. Verbitsky, The form boundedness criterion for the relativistic Schrödinger operator, preprint math-ph/0309031, to appear in Ann. Inst. Fourier.

[RSi] M. Reed and B. Simon, Methods of Modern Mathematical Physics. II: Fourier Analysis, Self-Adjointness, Academic Press, New York-London, 1975.

[RSS] G. V. Rozenblum, M.A. Shubin, and M.Z. Solomyak, Spectral Theory of Differential Operators, Encyclopaedia of Math. Sci., 64. Partial Differential Equations VII. Ed. M.A. Shubin. Springer-Verlag, Berlin-Heidelberg, 1994.

[Sch] M. Schechter, Operator Methods in Quantum Mechanics, Dover Publications, Mineola, NY, 2002.

[Sim] B. Simon, Schrödinger semigroups, Bull. Amer. Math. Soc. 7 (1982), 447-526.

[St1] E. M. Stein, Singular Integrals and Differentiability Properties of Functions, Princeton University Press, Princeton, New Jersey, 1970. 93

[St2] E. M. Stein, Harmonic Analysis: Real-Variable Methods, Orthogonality, and Oscillatory Integrals, Princeton University Press, Princeton, New Jersey, 1993.

[Tri] H. Triebel, The Structure of Functions. Monographs in Mathematics, 97. Birkhäuser Verlag, Basel, 2001.

[Tru] N. S. Trudinger, Linear elliptic operators with measurable coefficients, Ann. Scuola Norm. Sup. Pisa 27 (1973), 265-308.

[V1] I. E. Verbitsky, Imbedding and multiplier theorems for discrete Littlewood-Paley spaces, Pacific J. Math., 176 (1996), 529-556.

[V2] I. E. Verbitsky, Nonlinear potentials and trace inequalities, The Maz'ya Anniversary Collection. Eds. J. Rossmann, P. Takác, and G. Wildenhain. Operator Theory: Advances and Applications 110 (1999), 323-343. 
Department of Mathematics, Ohio State University, 231 West 18-th Ave., Columbus, OH 43210, USA

Department of Mathematics, LinkÖping University, SE-581 83, LinkÖping, SWEDEN

E-mail address: vlmaz@mai.liu.se

Department of Mathematics, University of Missouri, Columbia, MO 65211 , USA

E-mail address: igor@math.missouri.edu 